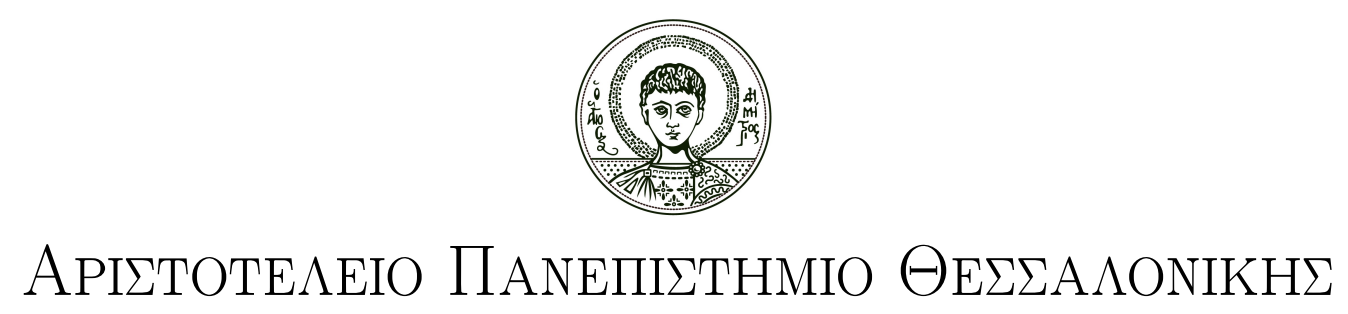

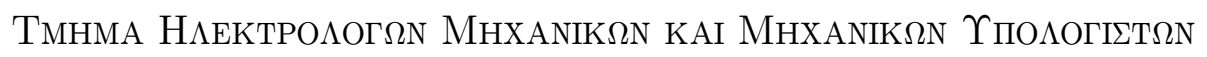

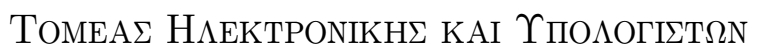

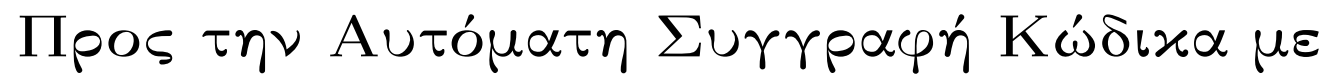

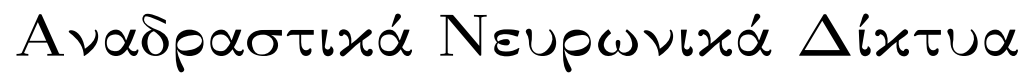

\author{
$\triangle$ IП $\Lambda$ MATIKH ЕРГАЕIA

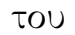

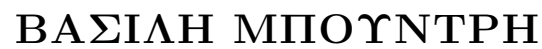

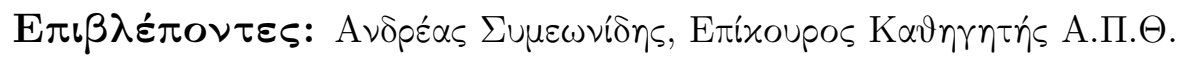

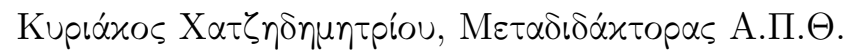

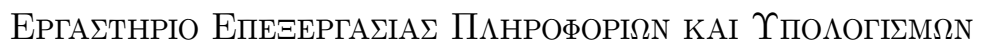

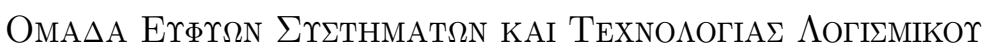

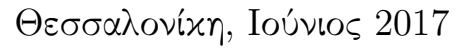





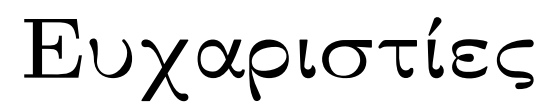

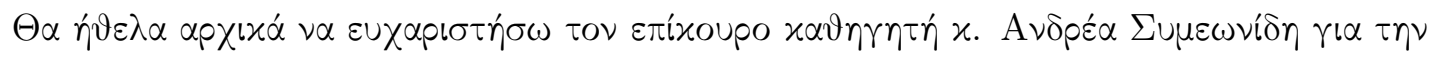

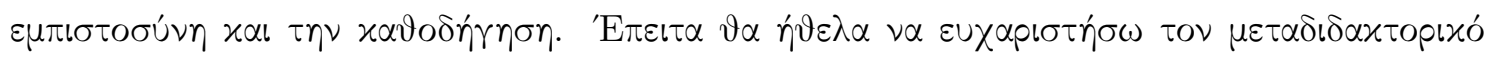

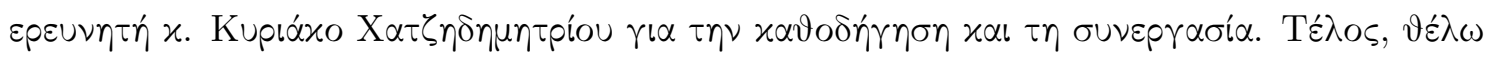

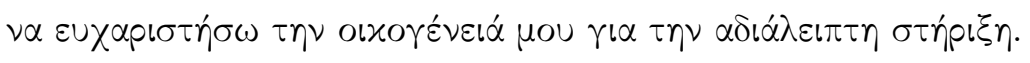





\section{$\Pi \varepsilon p i \lambda \eta \psi \eta$}

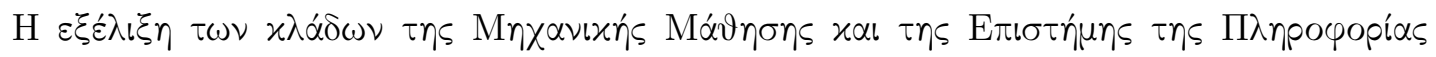

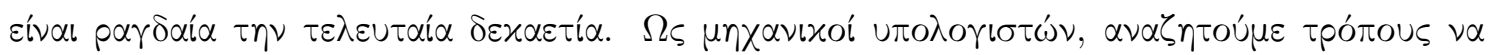

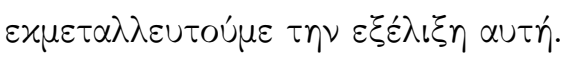

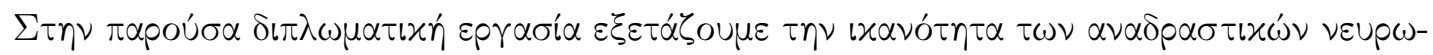

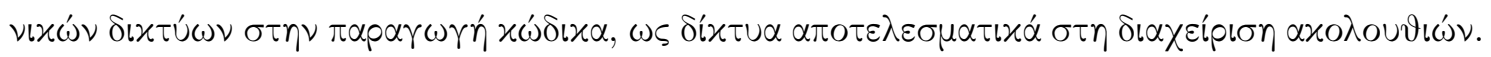

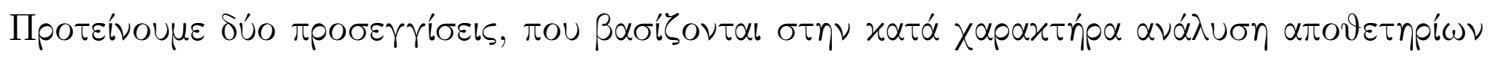

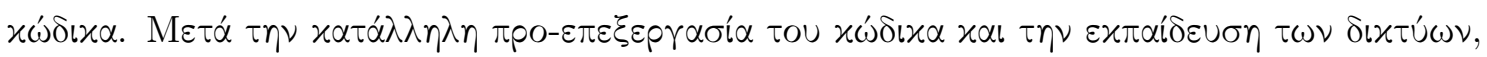

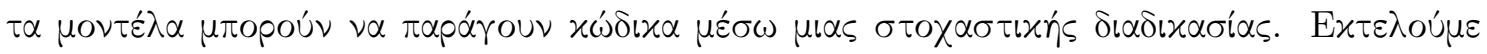

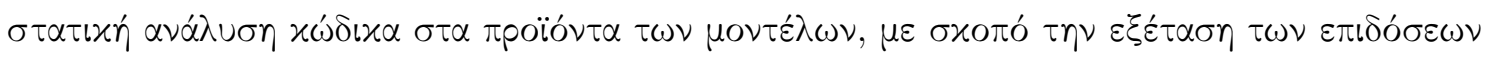

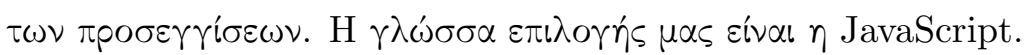

$\mathrm{H} \alpha \nu \alpha \dot{\lambda} \cup \sigma \eta \eta$

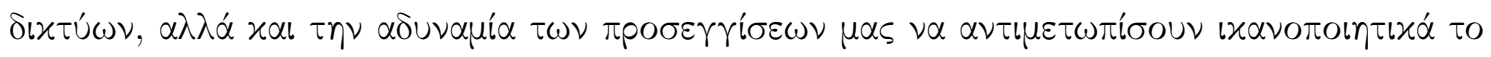

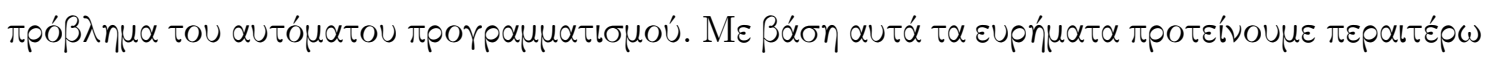

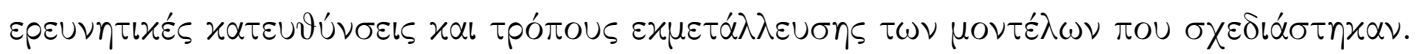

\section{$\Lambda \varepsilon ́ \xi \varepsilon \iota \varsigma \mathrm{K} \lambda \varepsilon \iota \delta \iota \alpha$}

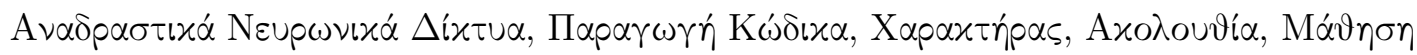





\title{
Towards Source Code Generation with Recurrent Neural Networks
}

\begin{abstract}
The evolution of Machine Learning and Data Science disciplines has been rapid during the last decade. As computer engineers, we are looking for ways to take advantage of this evolution.

In this diploma thesis we examine the potential of recurrent neural networks to generate source code, given their effectiveness at handling sequences. We propose two approaches, based on per-character analysis of software repositories. Following appropriate code preprocessing and network training, models generate source code through a stochastic process. We perform static code analysis on model products, in order to examine the performance of the approaches. We have applied our approach on the JavaScript Language.

The analysis shows the great representational power of the recurrent neural networks, but also the inability of our approaches to satisfactorily address the problem of automatic programming. Based on these findings, we propose further research directions and ways of exploiting the models that were designed.
\end{abstract}

\section{Keywords}

Recurrent Neural Networks, Source Code Generation, Character, Sequence, Learning

Vasilis Bountris

mvasilis@auth.gr

Aristotle University of Thessaloniki

June 2017 



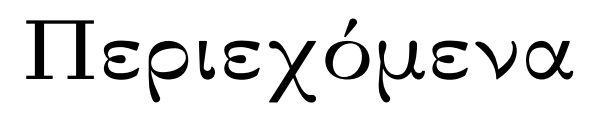

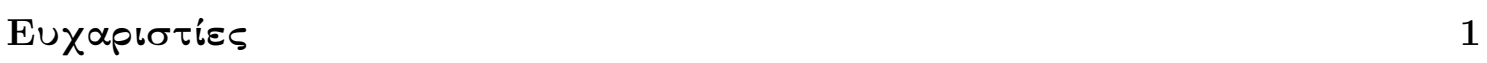

Пвріл $\eta \psi \eta$

$\begin{array}{ll}\text { Abstract } & 5\end{array}$

$\begin{array}{lr}\Pi \varepsilon \rho\llcorner\chi o ́ \mu \varepsilon \nu \alpha & 8\end{array}$

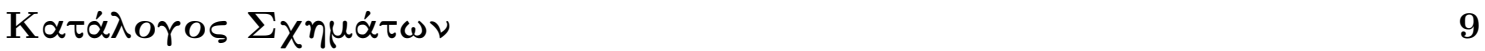

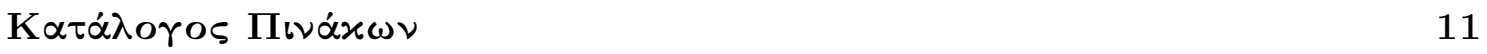

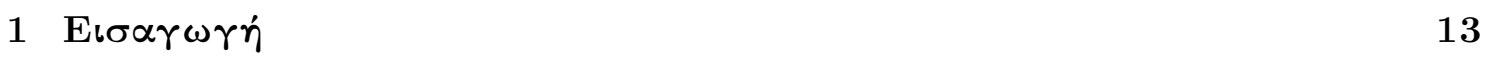

1.1 Kinntpo . . . . . . . . . . . . . . . . . . . . . . . . 14

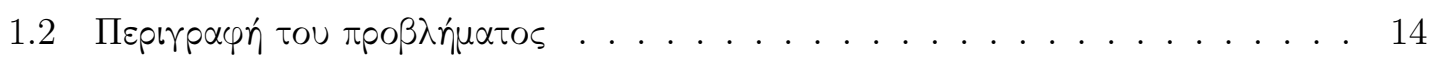

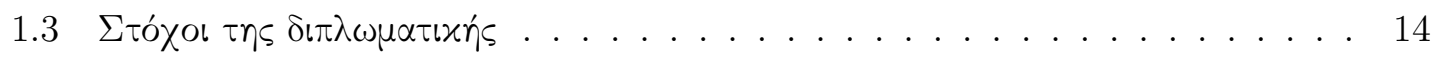

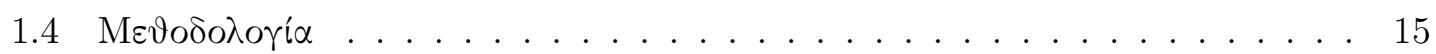

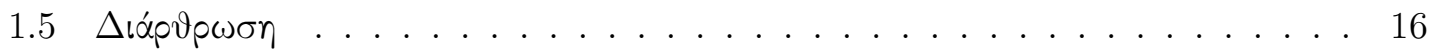

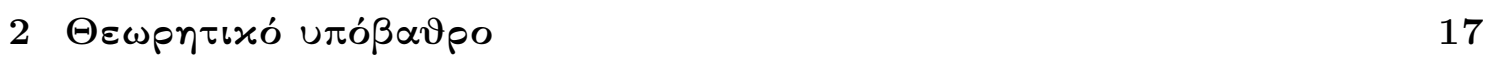

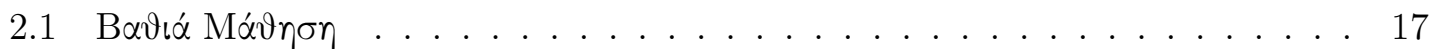

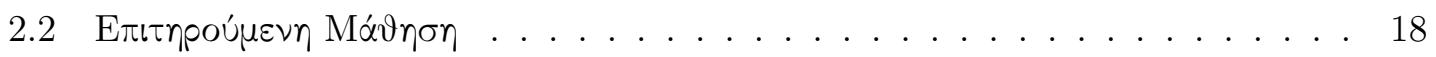

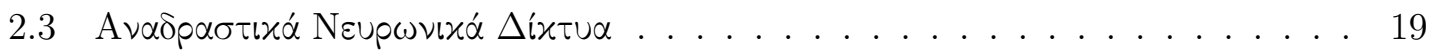

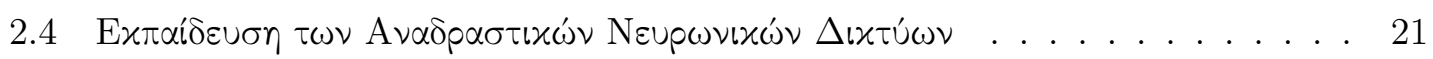

2.4.1 Long Short-Term Memory Units . . . . . . . . . . . . . . . . . . . . 21

2.4.2 Truncated Backpropagaion Through Time . . . . . . . . . . . . 22

2.4 .3 Dropout . . . . . . . . . . . . . . . . . . . . 23

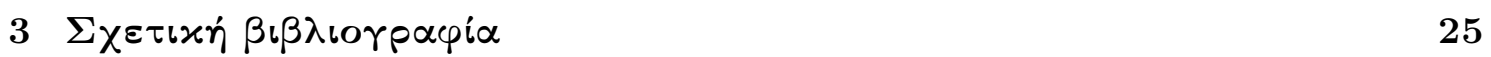

3.1 Generating Sequence with Recurrent Neural Networks . . . . . . . . . . . . 25

3.2 Inferring Algorithmic Patterns with Stack-Augmented Recurrent Nets . . . 26

3.3 A Synthetic Neural Model for General Purpose Code Generation . . . . . . 28

3.4 End-to-End Memory Networks . . . . . . . . . . . . . . . . . . . . 28 
3.5 Neuro Symbolic Program Synthesis . . . . . . . . . . . . . . . . . . . . . . 29

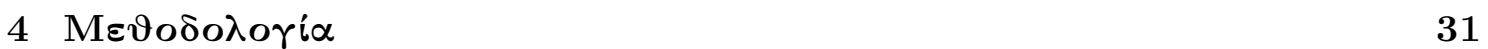

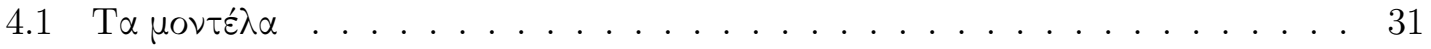

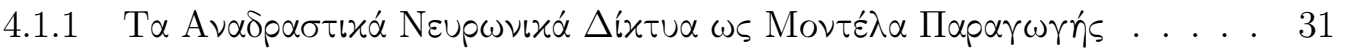

4.1 .2 Movté̀ ${ }_{0}$ char-rnn . . . . . . . . . . . . . . . . . . . 32

4.1 .3 Movtét入o labeled-char-rnn . . . . . . . . . . . . . . . . . . 32

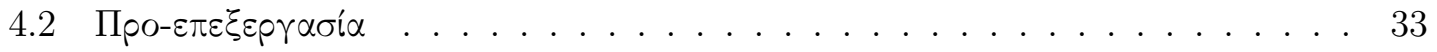

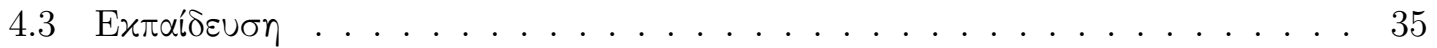

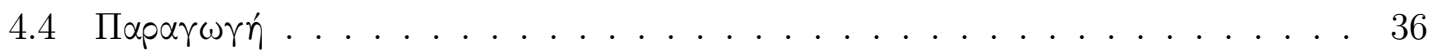

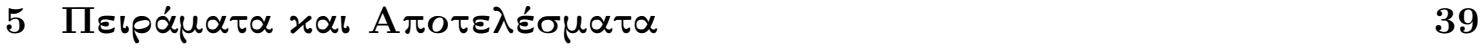

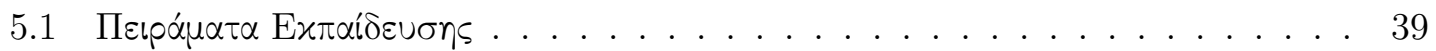

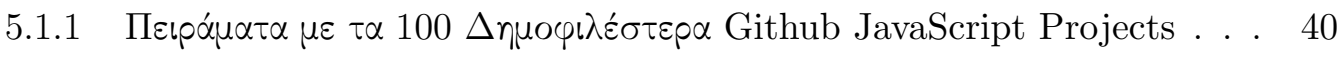

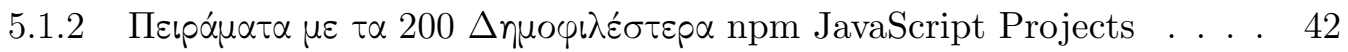

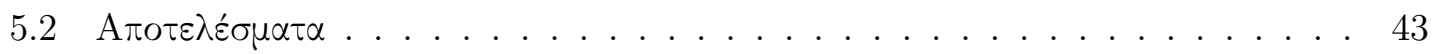

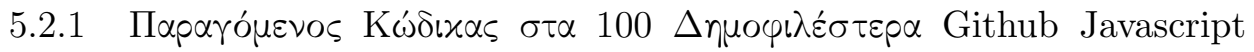
Projects . . . . . . . . . . . . . . . . . 44

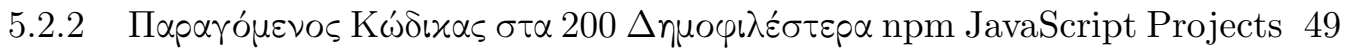

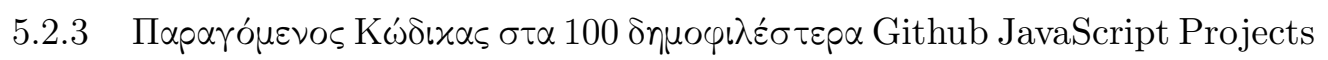

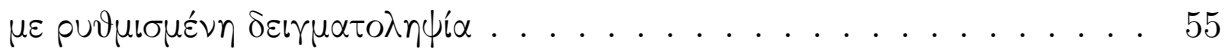

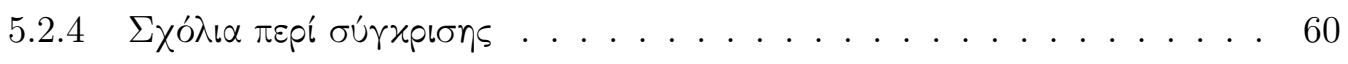

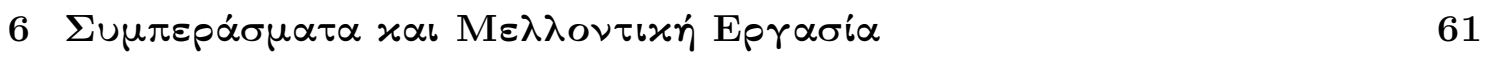

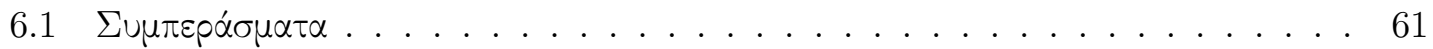

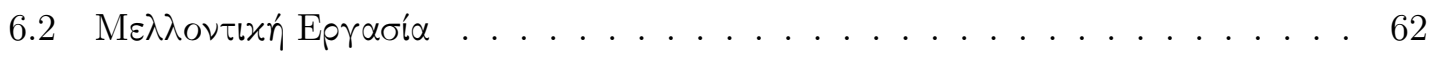

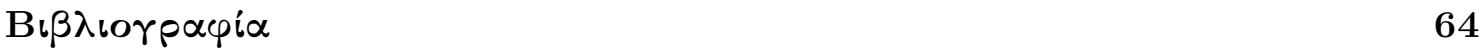




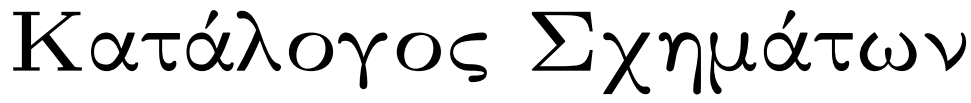

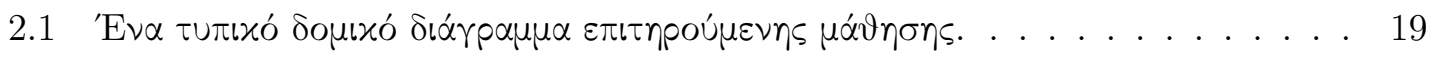

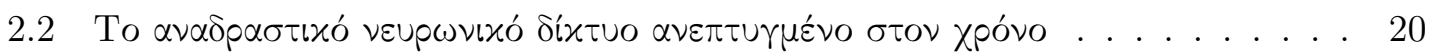

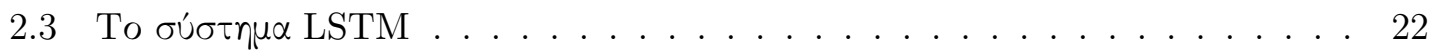

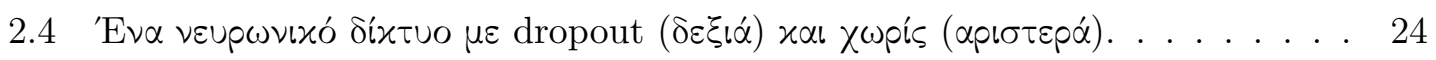

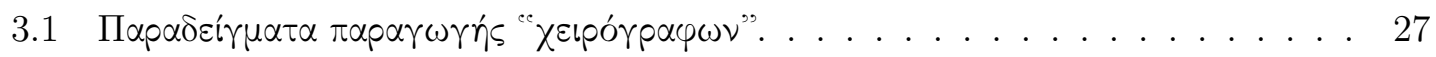

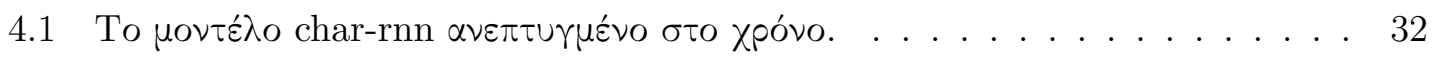

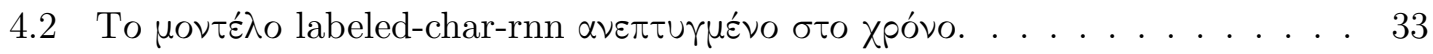

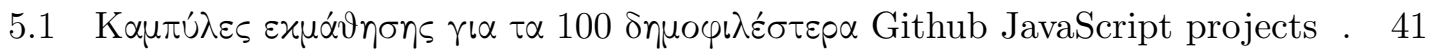

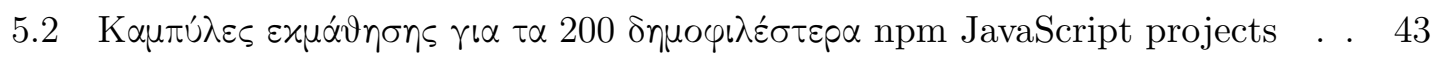

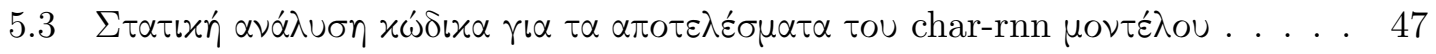

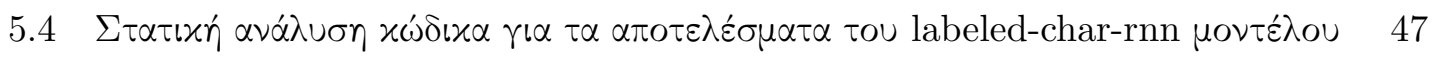

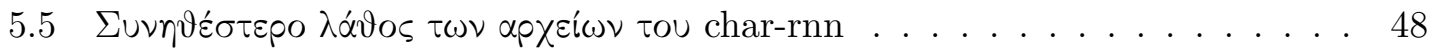

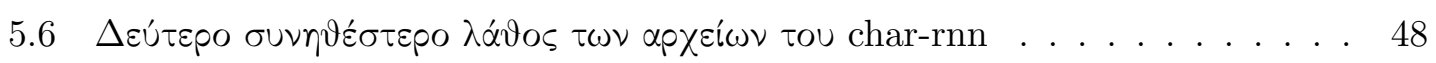

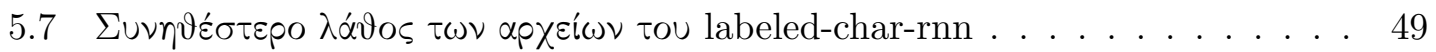

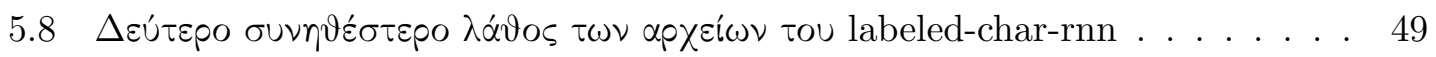

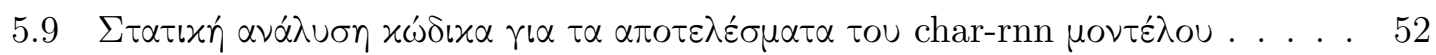

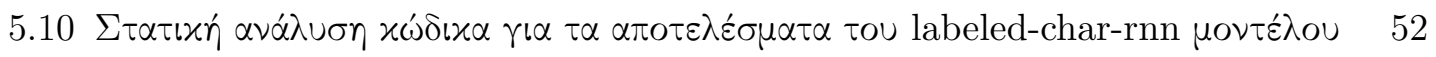

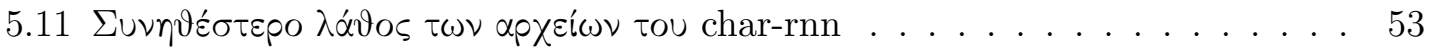

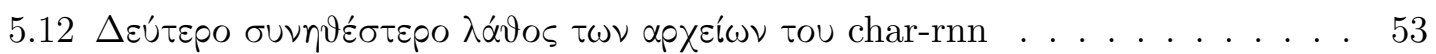

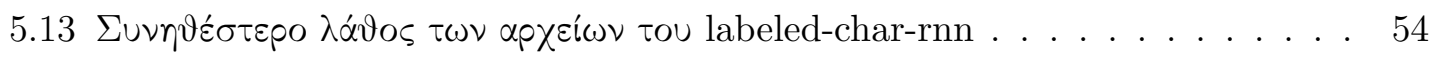

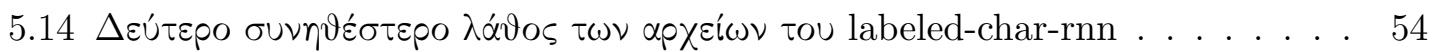

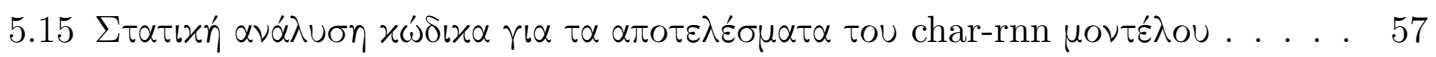

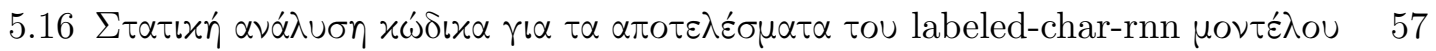

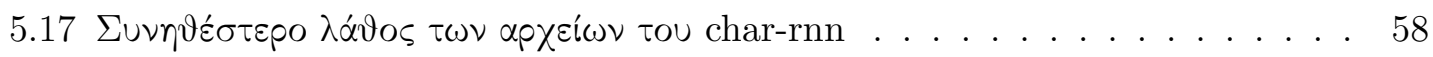

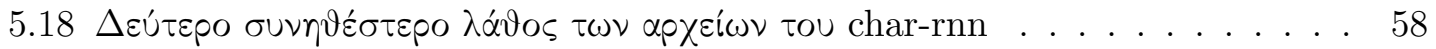

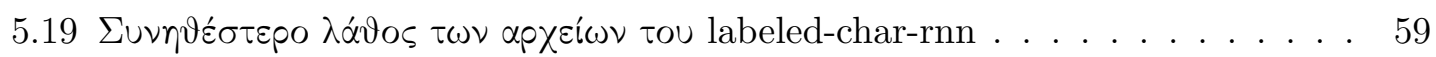

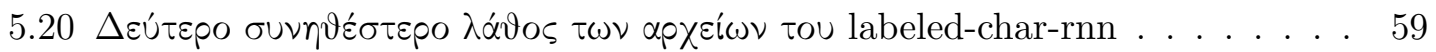





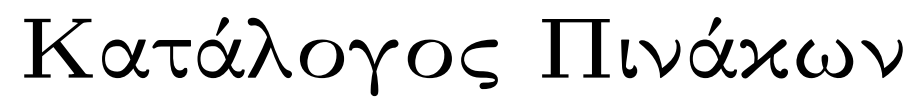

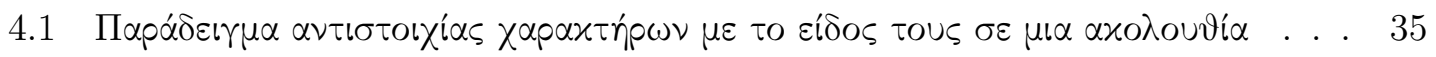

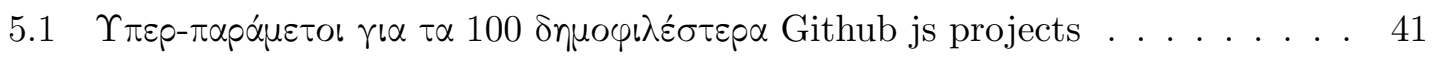

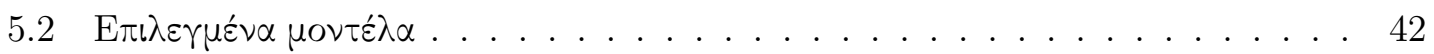

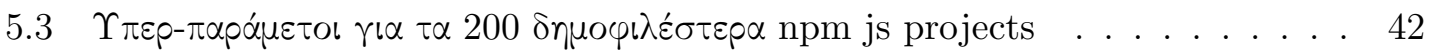

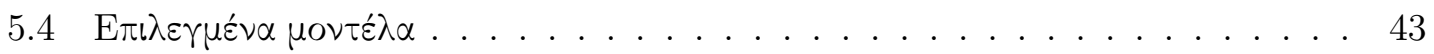

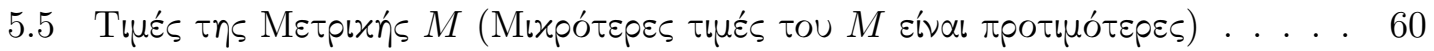





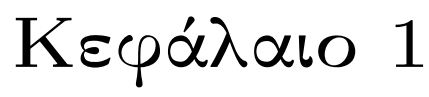

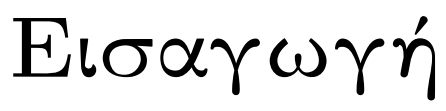

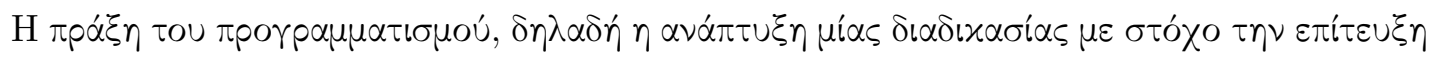

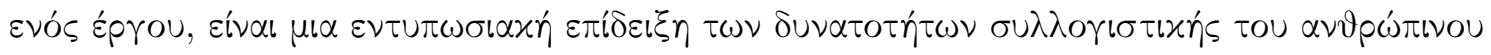

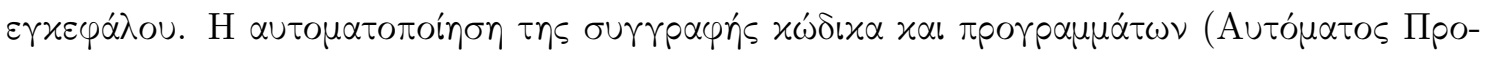

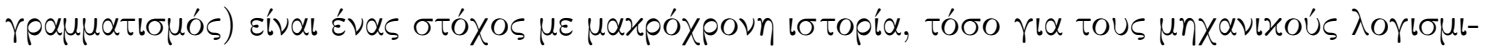

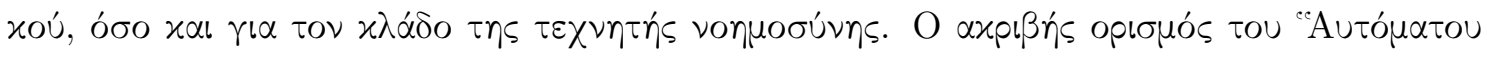

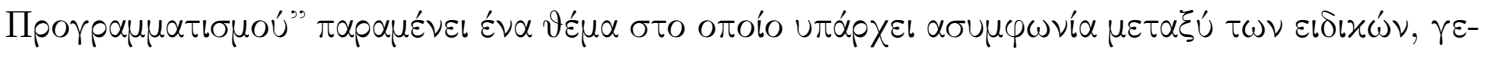

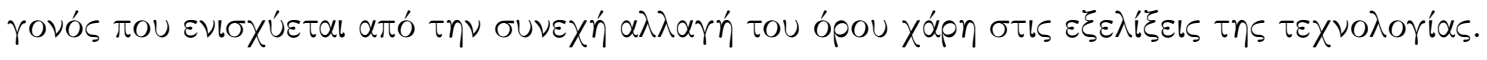

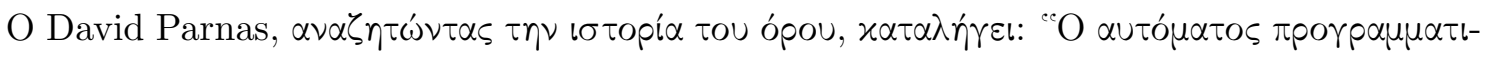

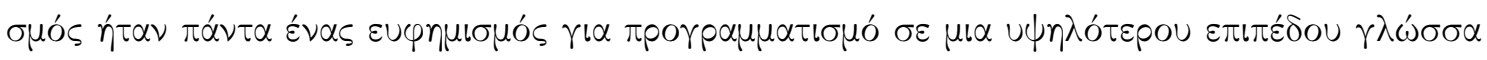

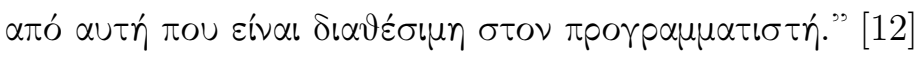

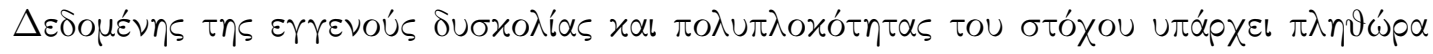

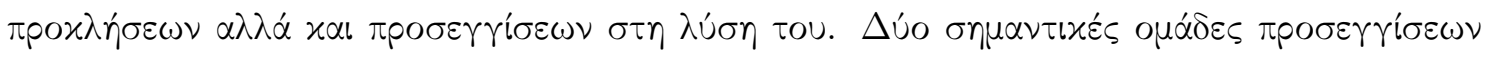
Eival [3], [14]:

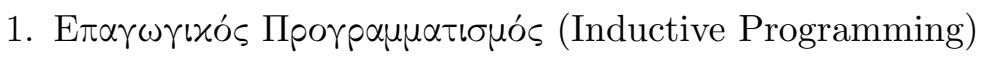

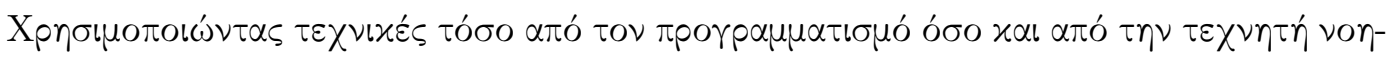

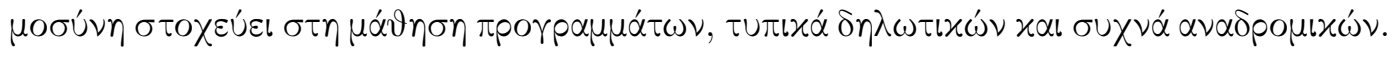

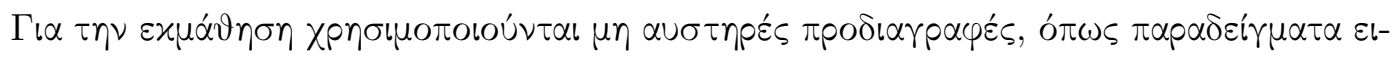

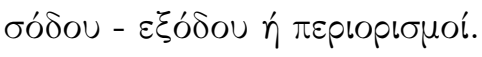

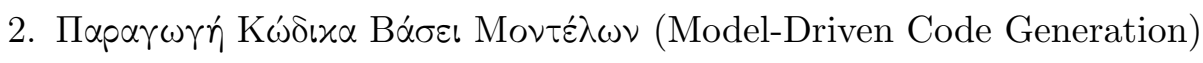

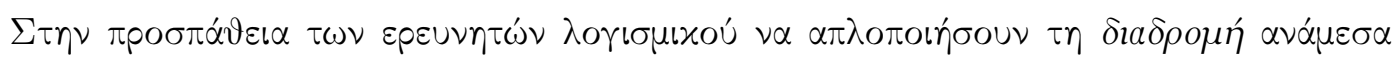

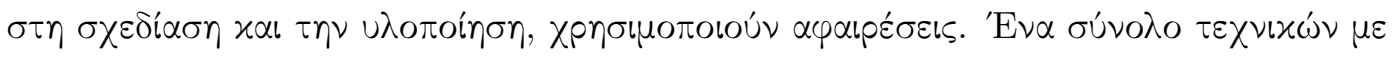

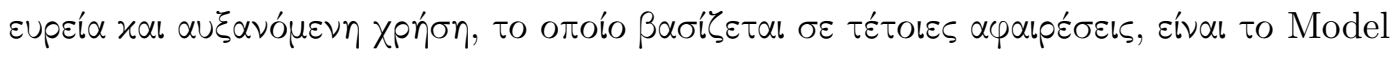

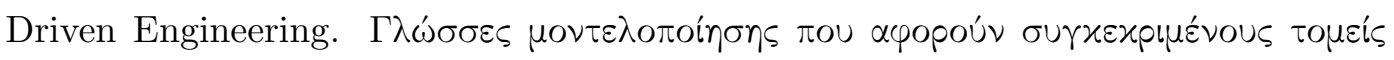

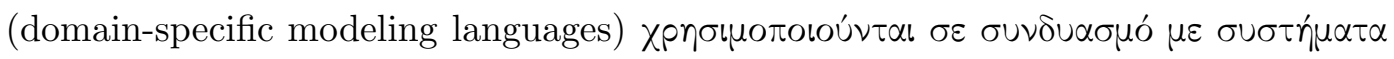

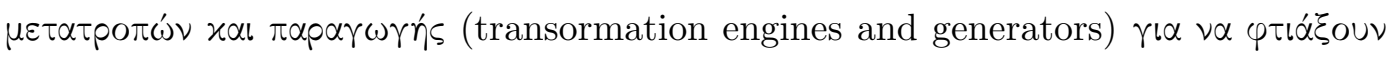

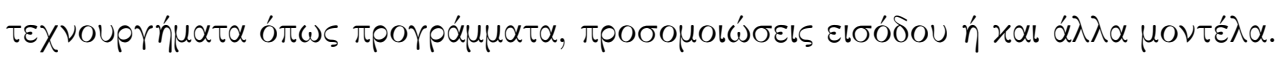

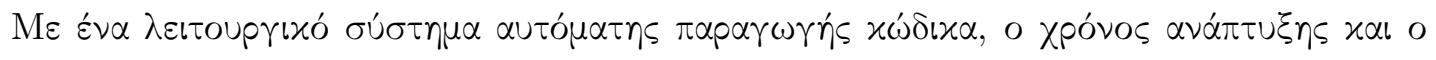




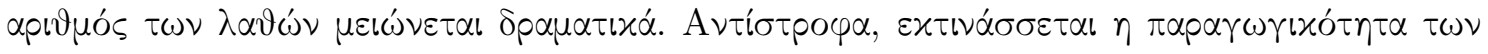

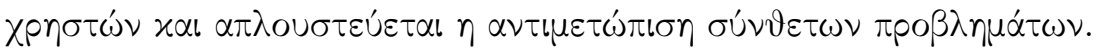

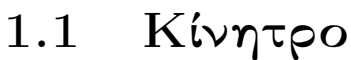

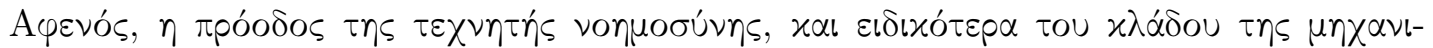

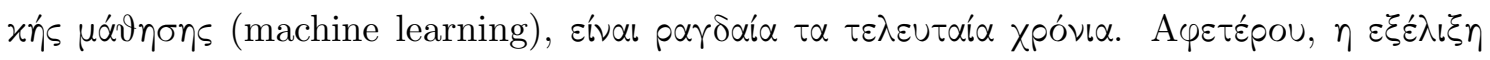

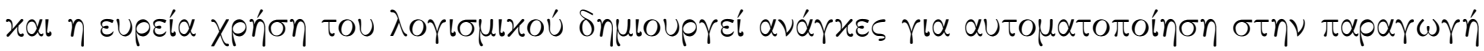

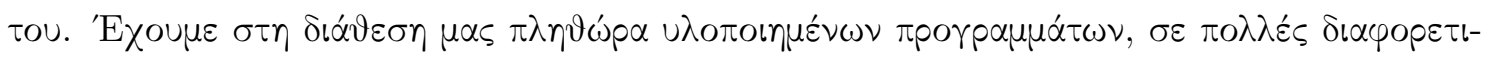

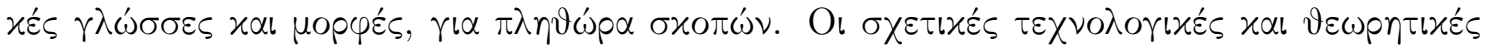

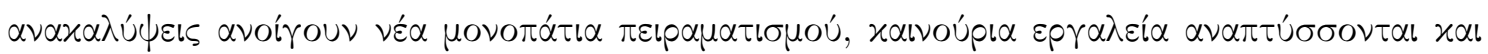

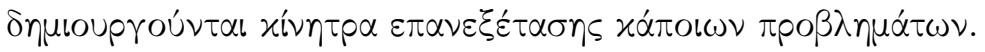

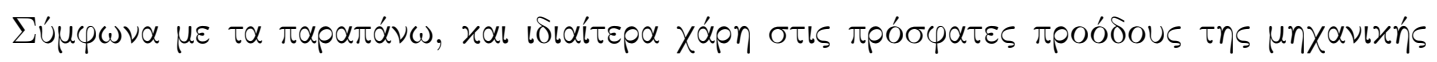

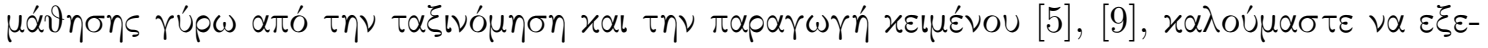

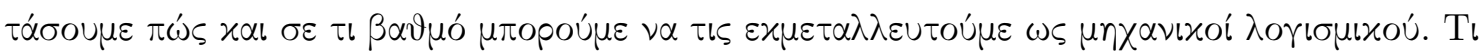

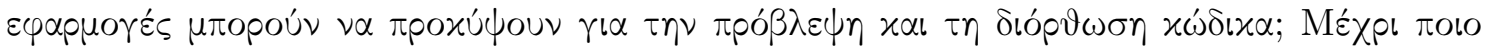

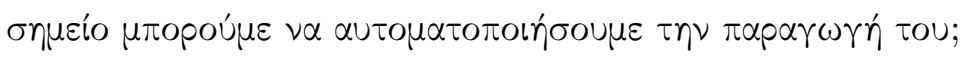

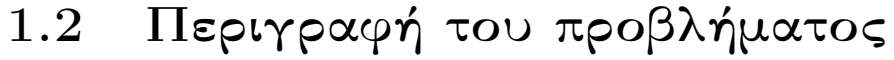

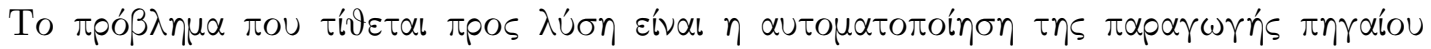

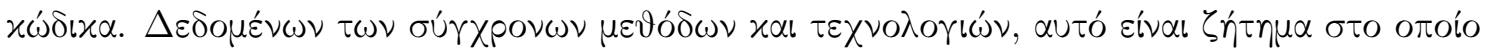

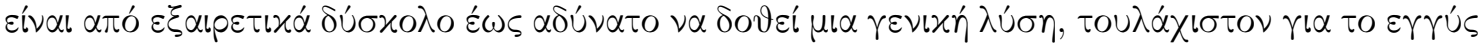

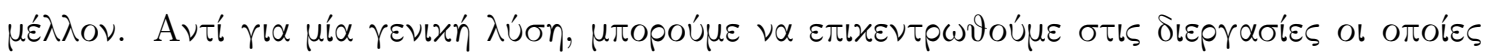

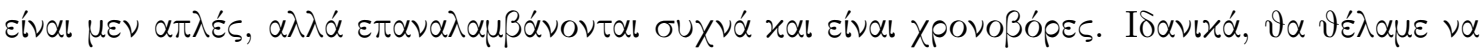

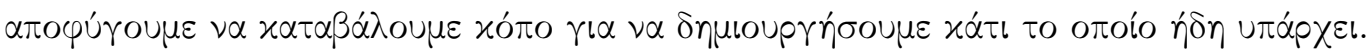

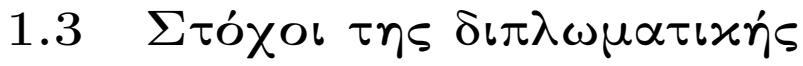

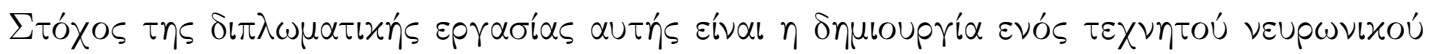

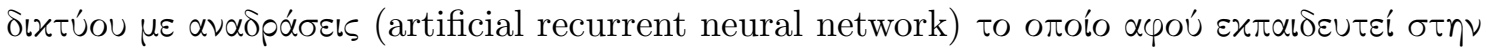

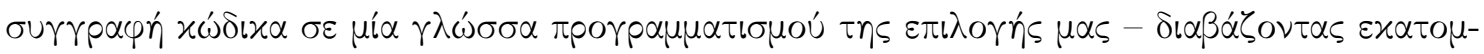

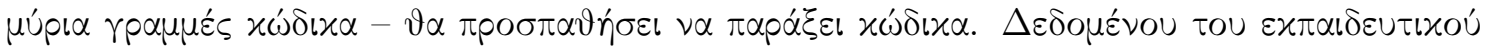

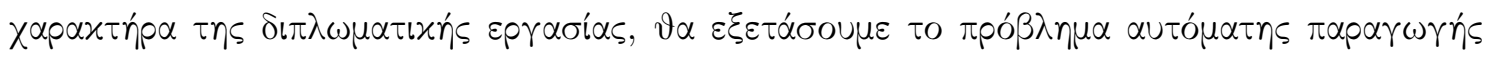

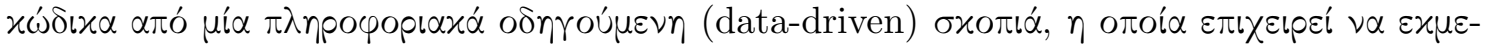

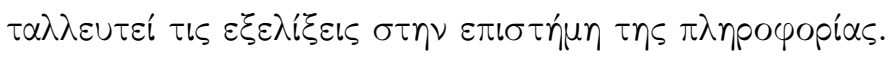

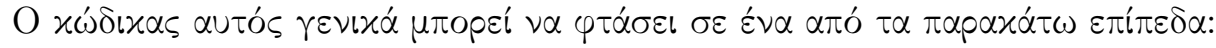

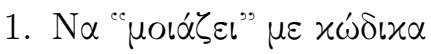

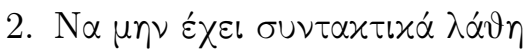


3. $\mathrm{N} \alpha \mu \pi \mathrm{\mu} \varepsilon \dot{\imath} \nu \alpha \mu \varepsilon \tau \alpha \varphi \rho \alpha \sigma \tau \varepsilon i ́$

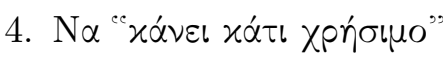

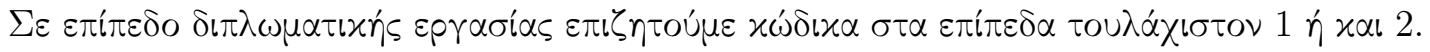

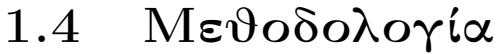

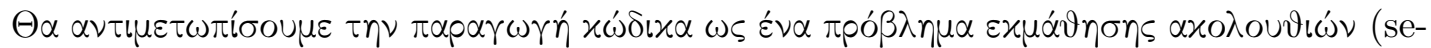

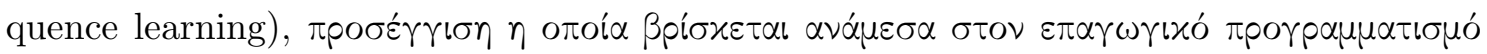

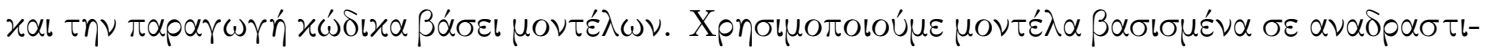

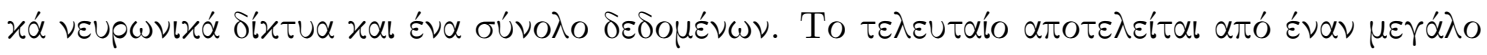

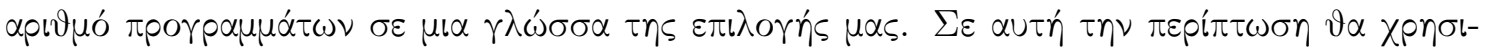

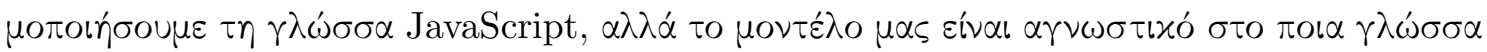

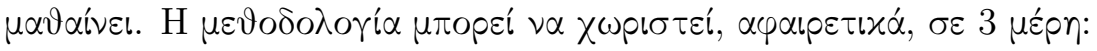

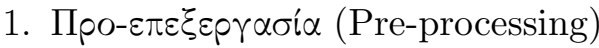

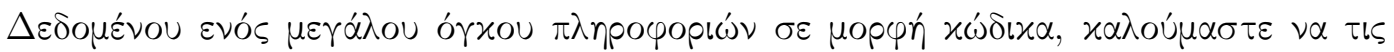

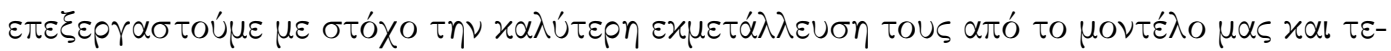

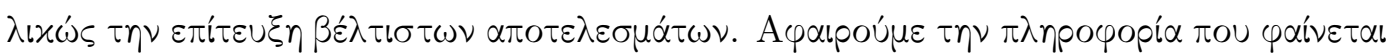

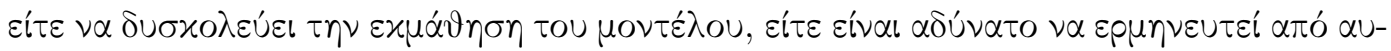

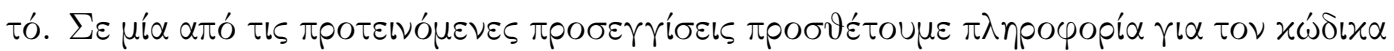

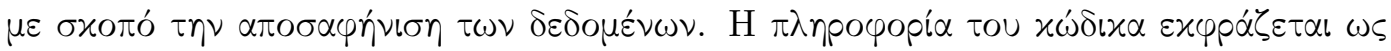

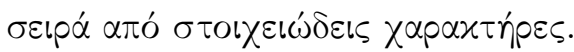

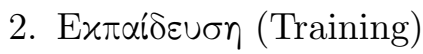

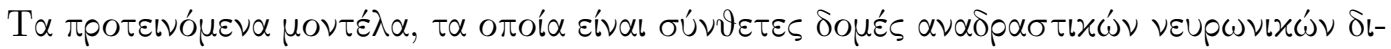

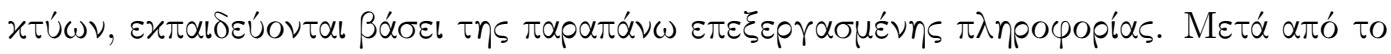

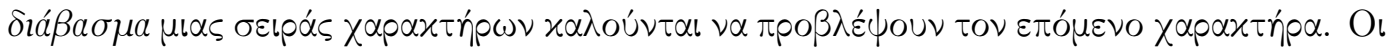

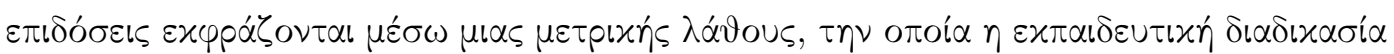

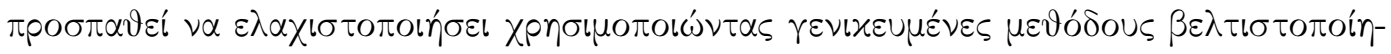
ons.

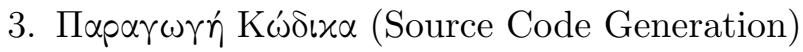

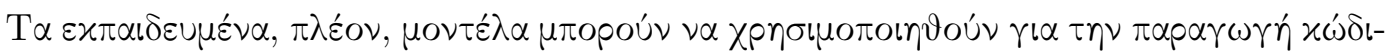

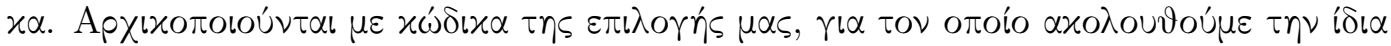

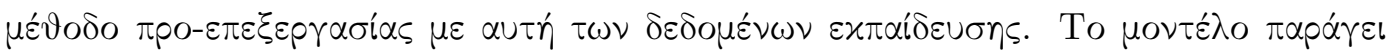

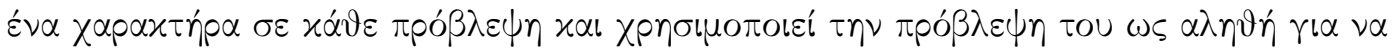

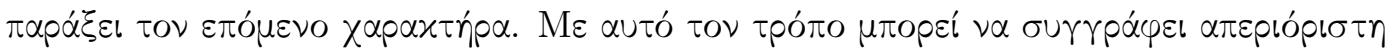
$\pi 0 \sigma o ́ \tau \eta \tau \alpha ~ x \omega ́ \delta \delta เ \alpha$. 


\section{$1.5 \Delta ı \alpha ́ \rho \vartheta \rho \omega \sigma \eta$}

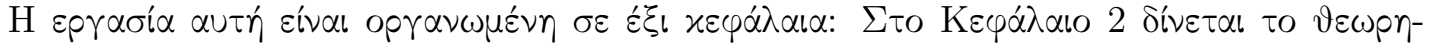

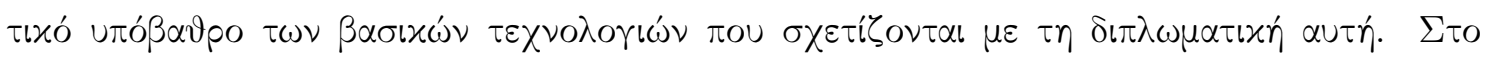

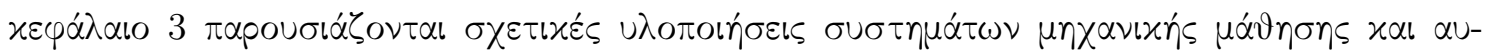

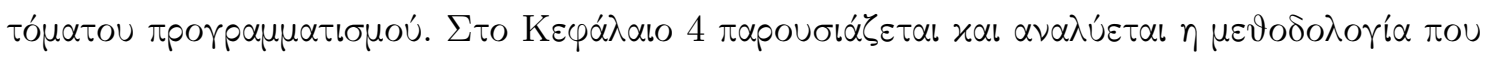

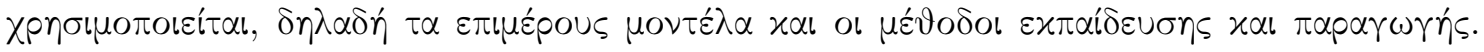

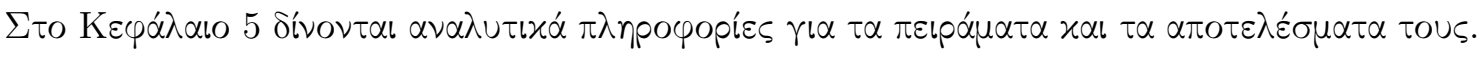

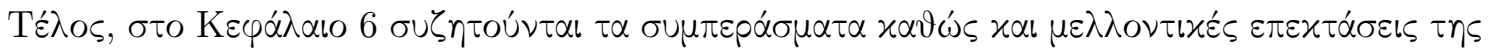

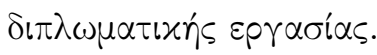




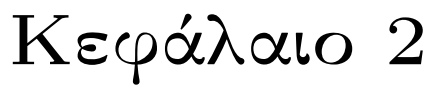

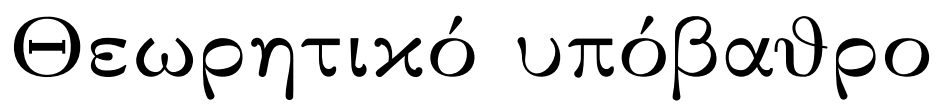

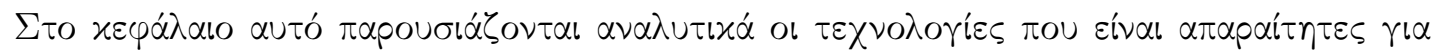

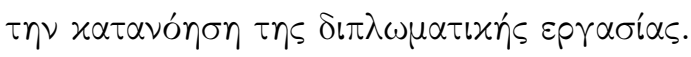

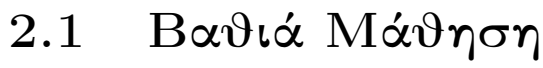

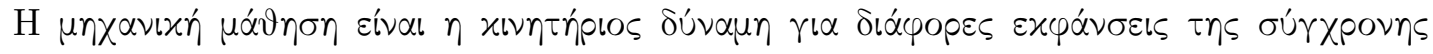

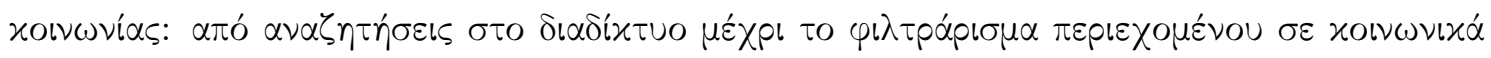

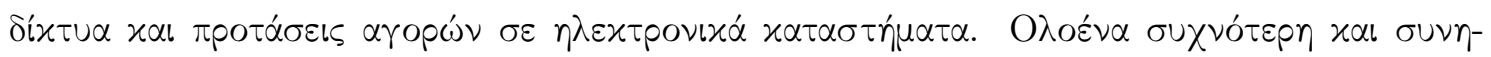

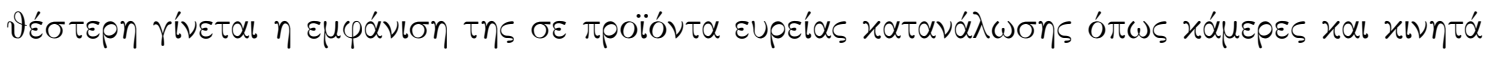

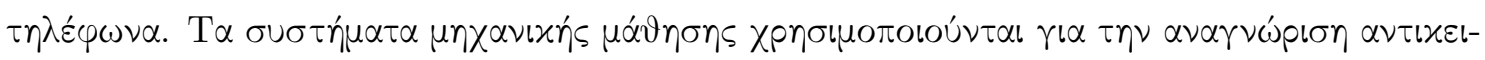

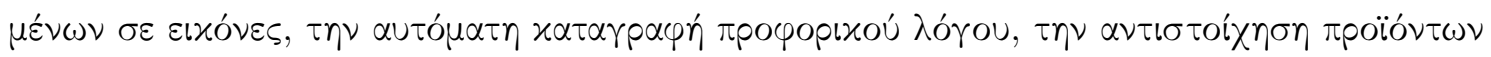

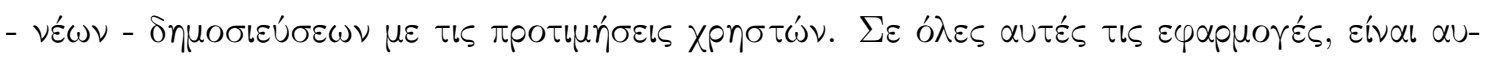

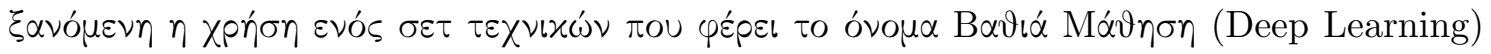
[8].

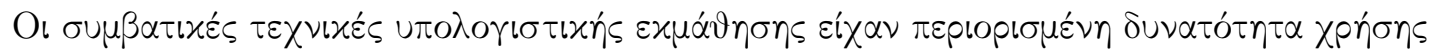

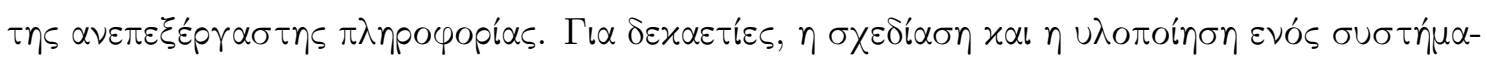

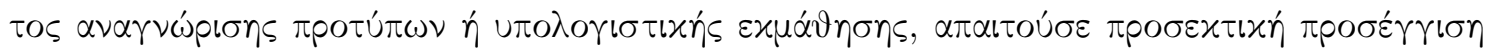

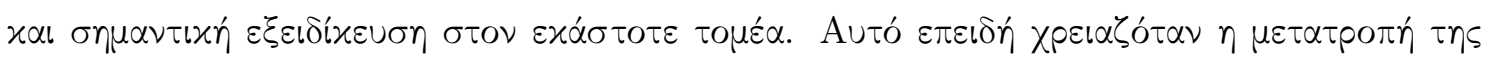

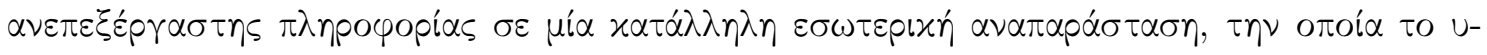

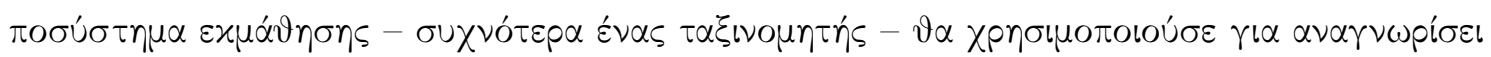

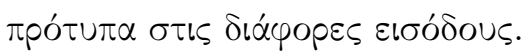

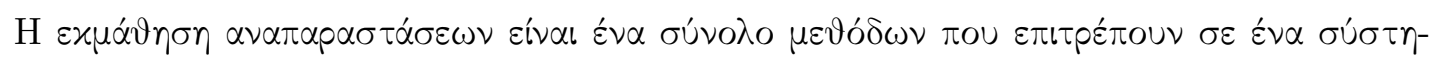

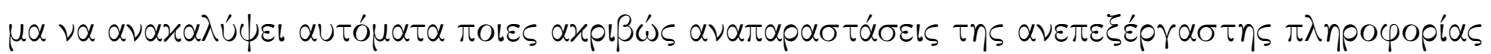

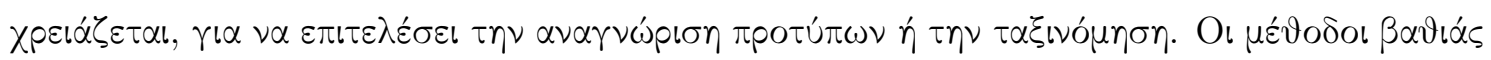

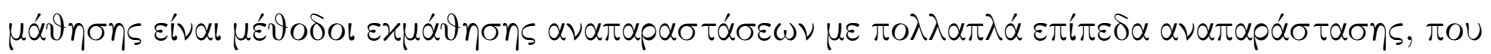

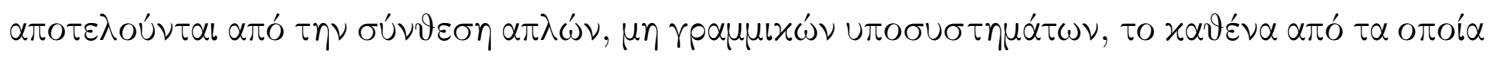

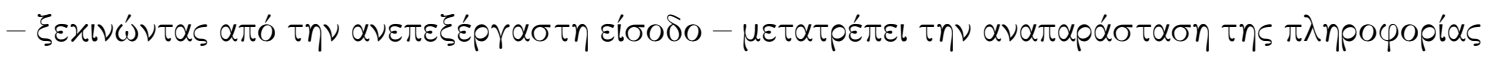

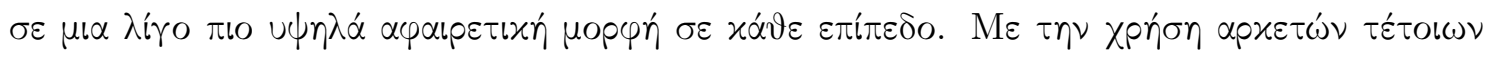

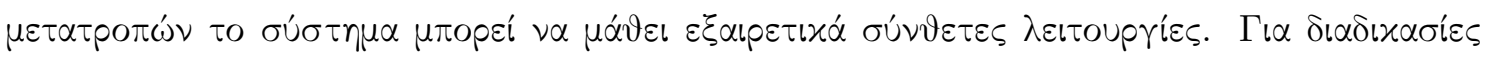




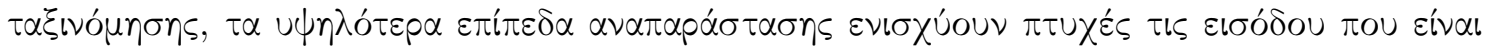

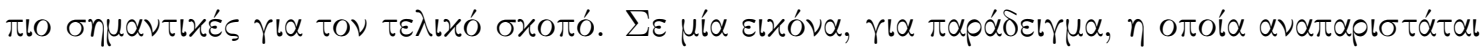

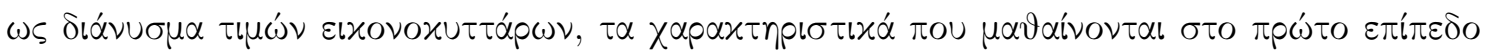

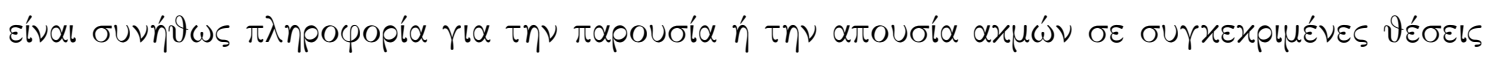

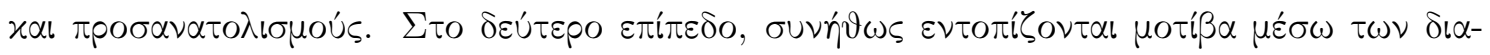

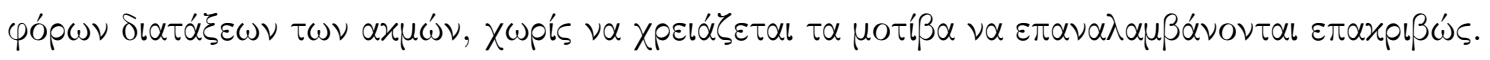

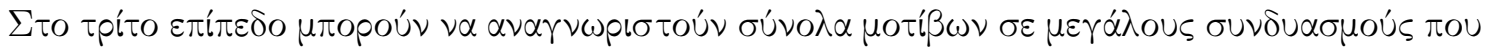

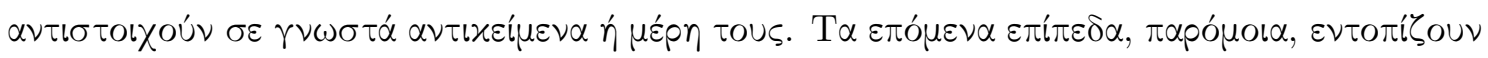

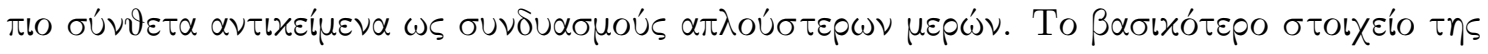

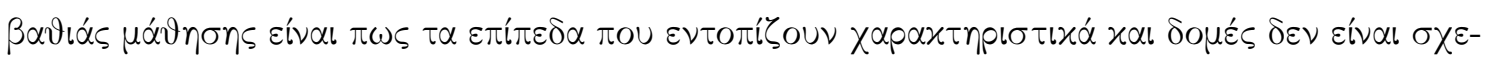

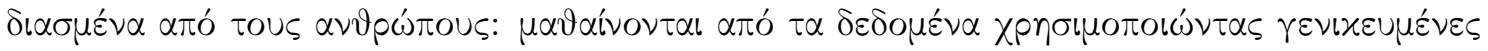

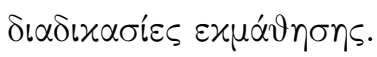

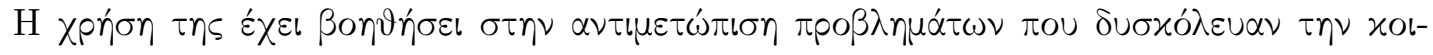

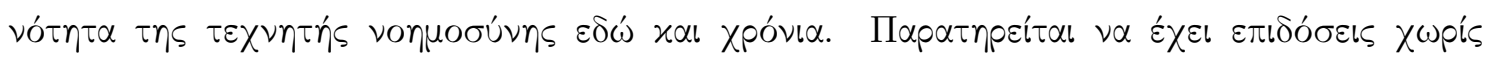

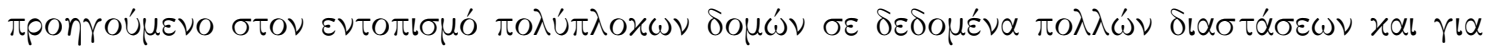

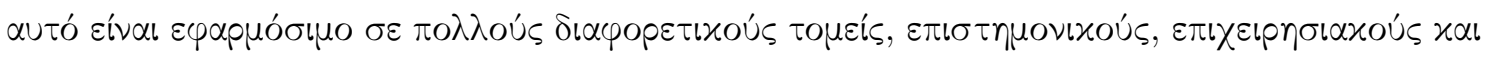

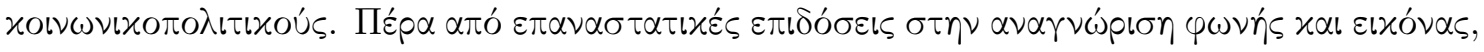

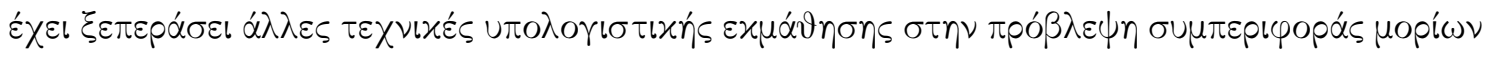

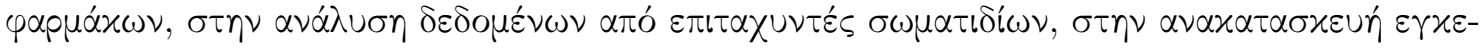

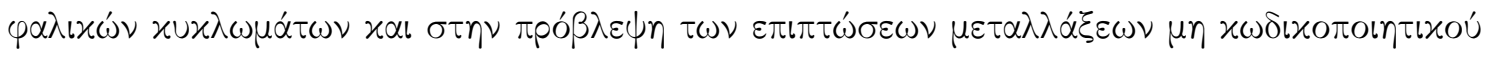

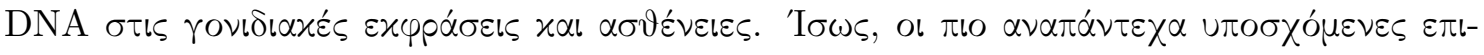

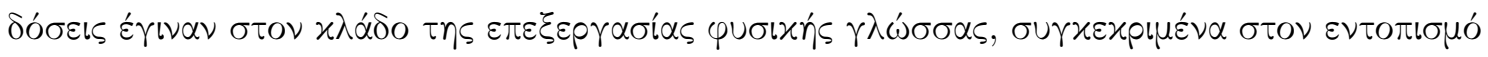

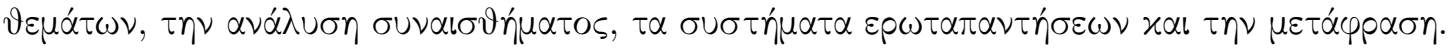

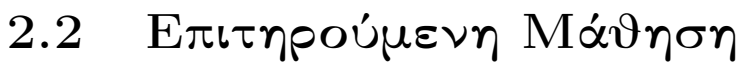

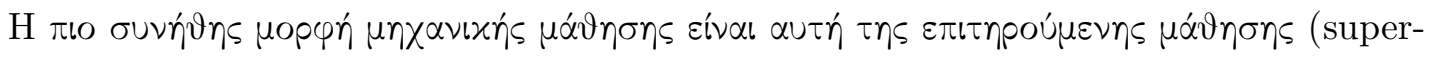

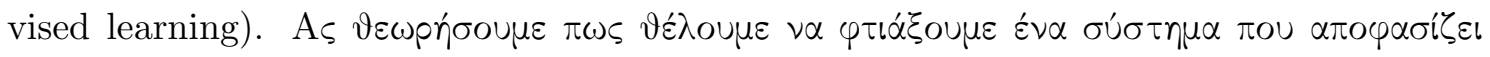

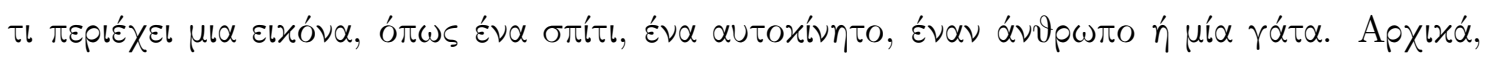

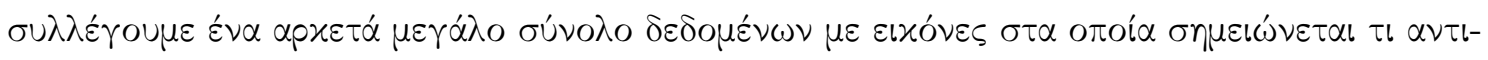

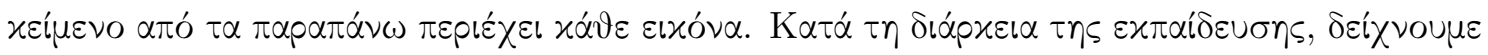

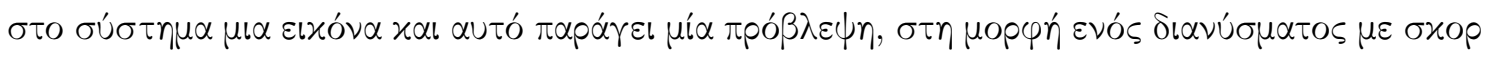

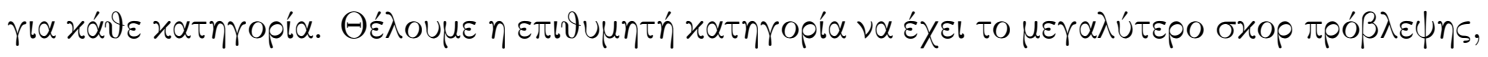

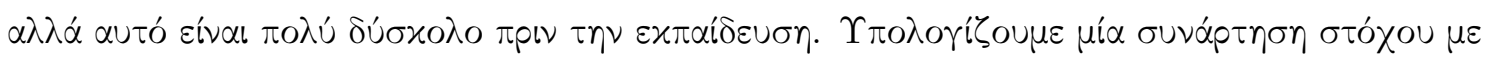

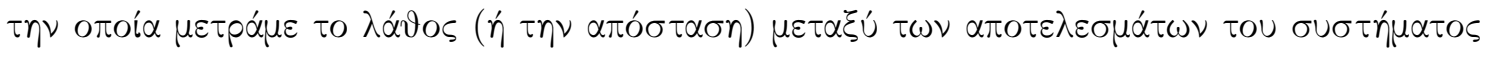

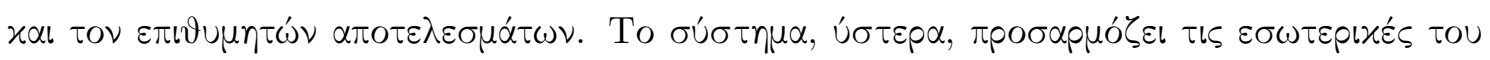

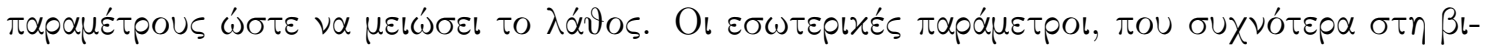

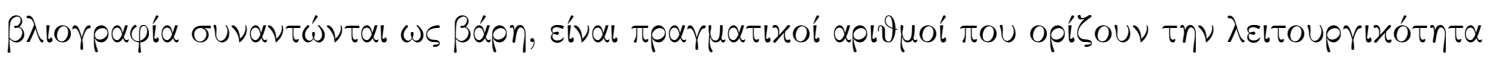

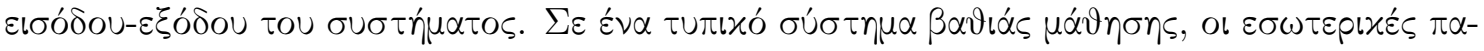

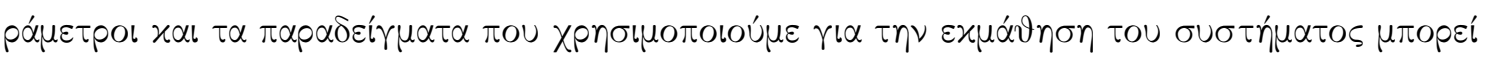

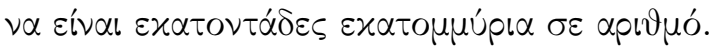




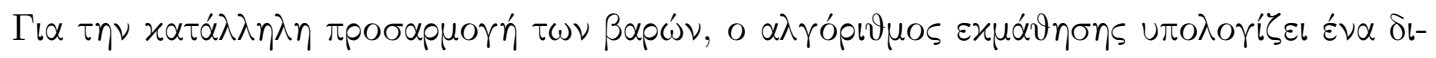

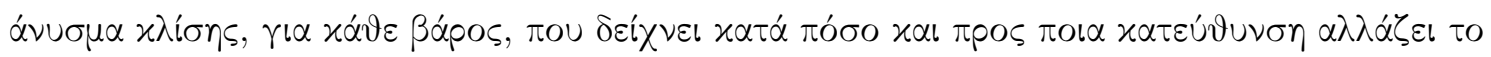

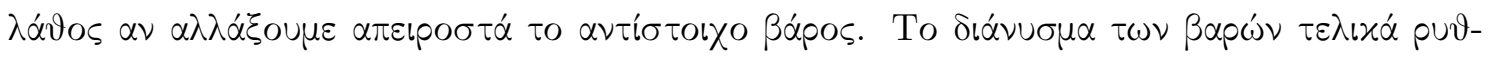

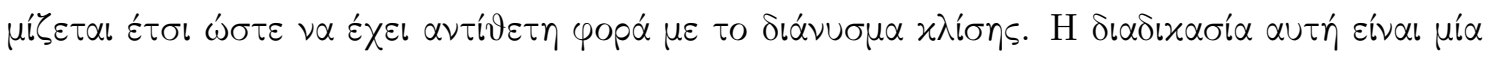

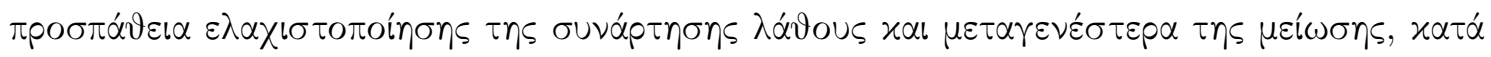

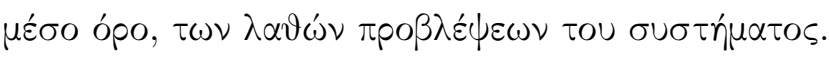

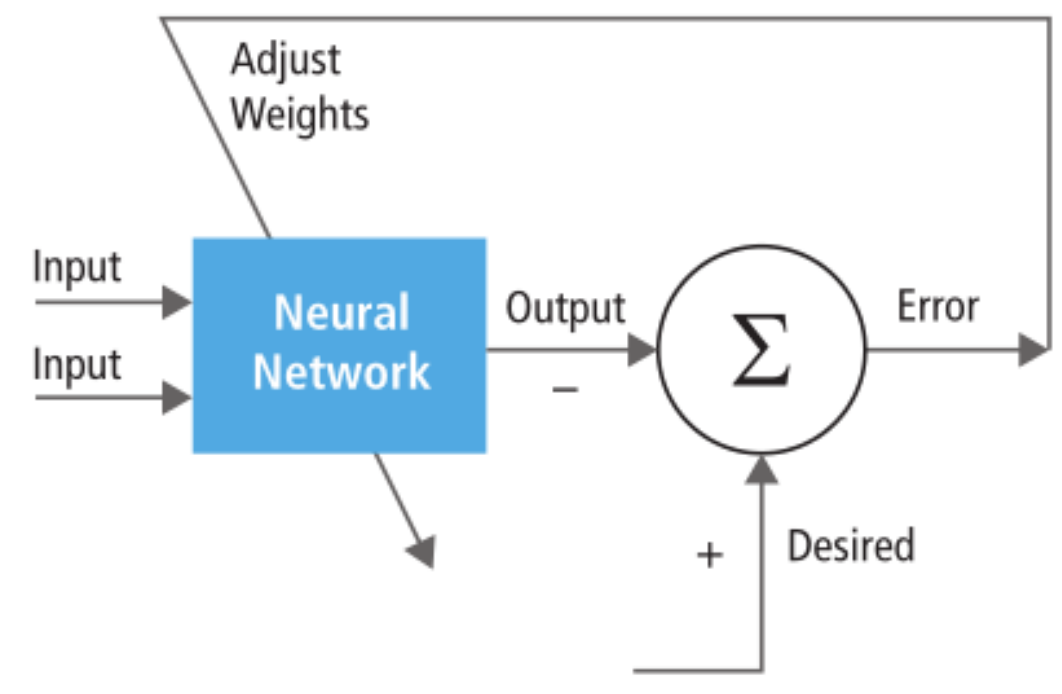

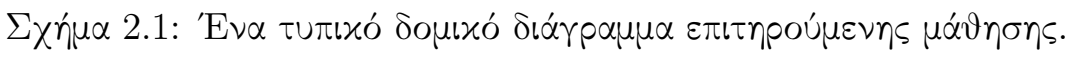

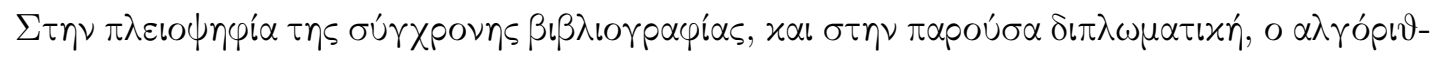

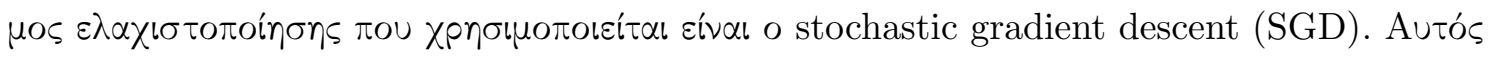

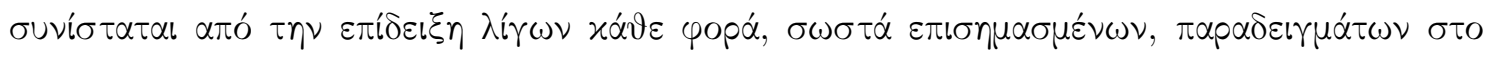

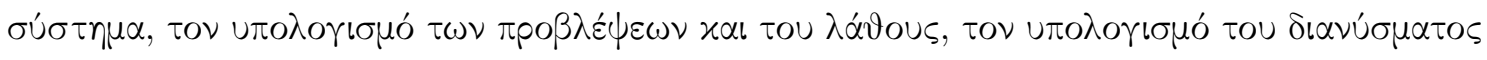

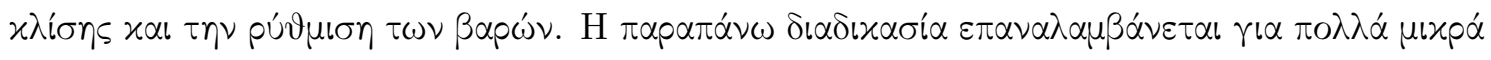

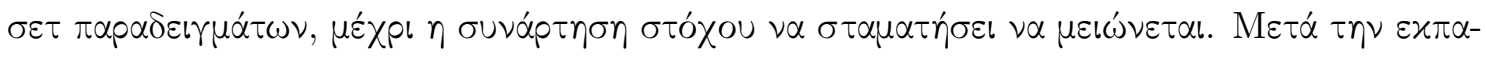

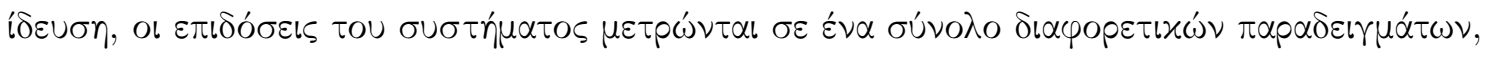

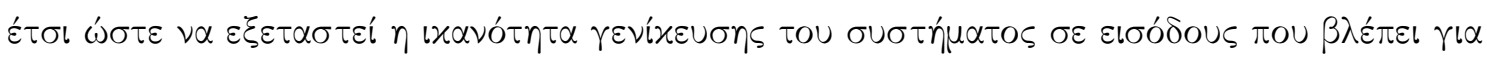

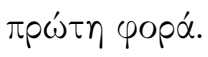

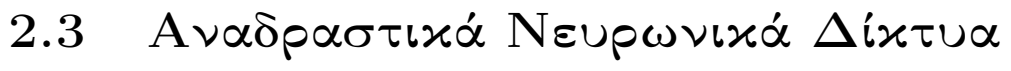

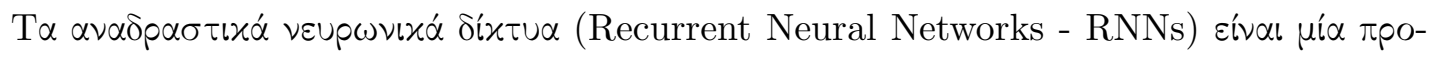

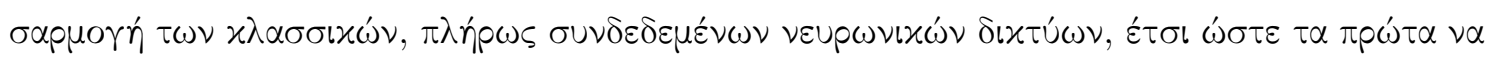

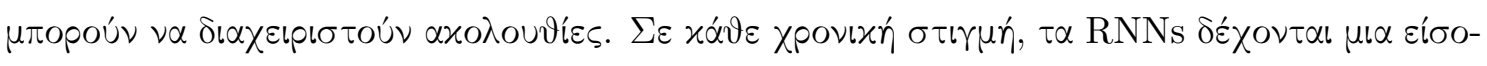

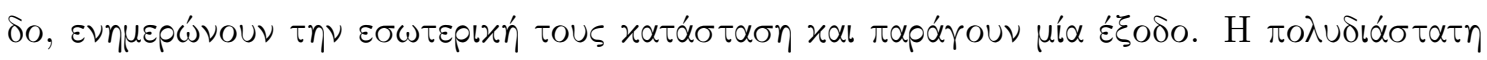

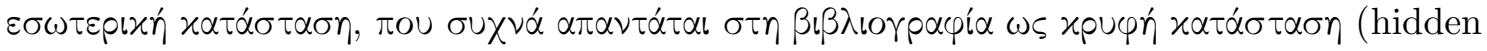

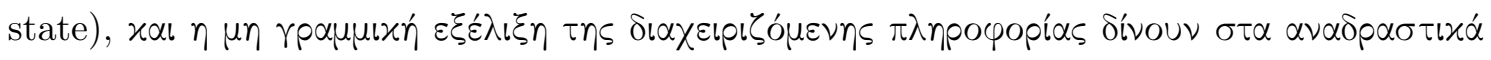

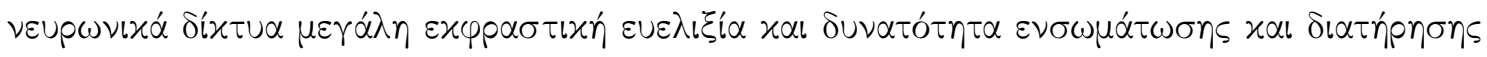




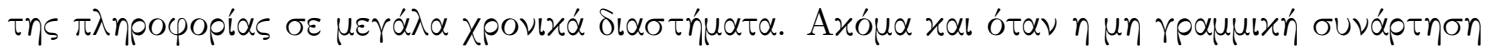

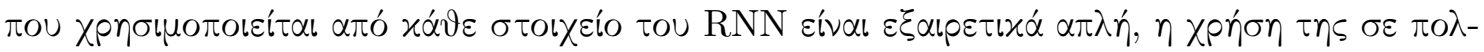

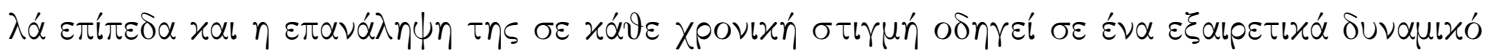
$\sigma \dot{\sigma} \sigma \eta \mu \alpha$.

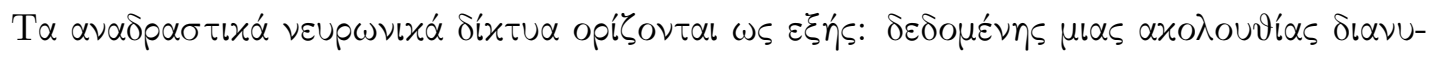

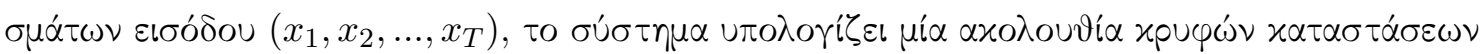

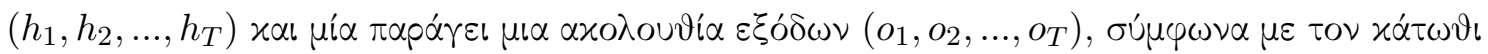
$\alpha \lambda \gamma o ́ p ı \vartheta \mu o:$

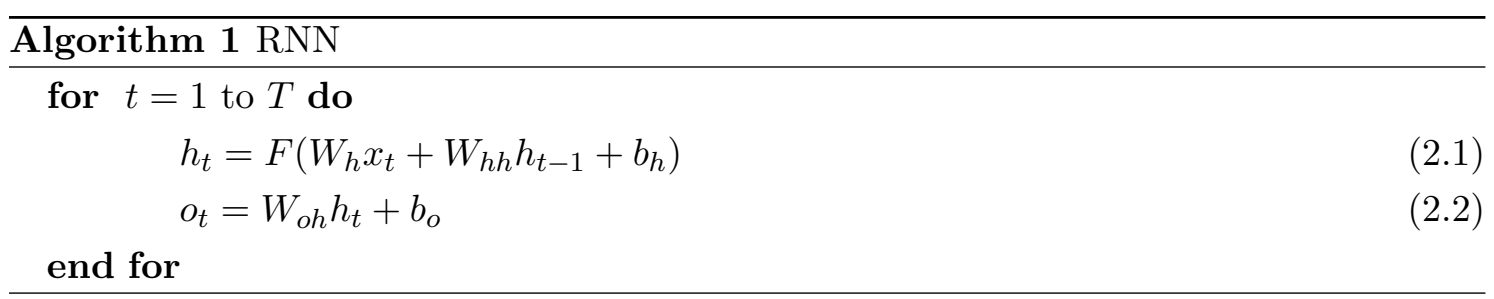

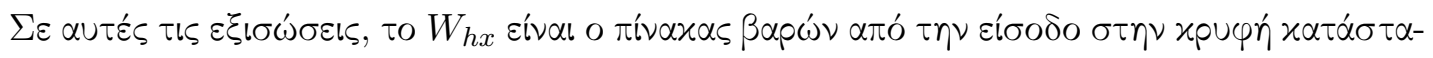

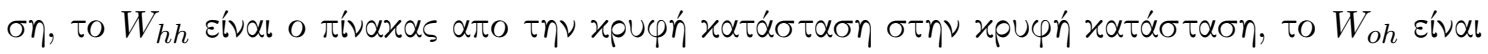

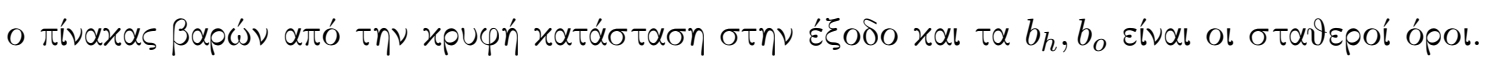

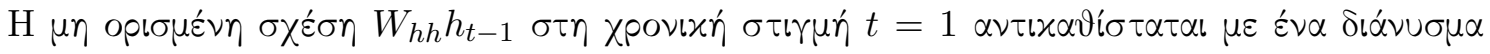

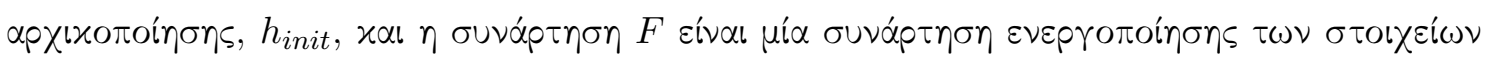

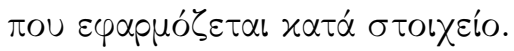

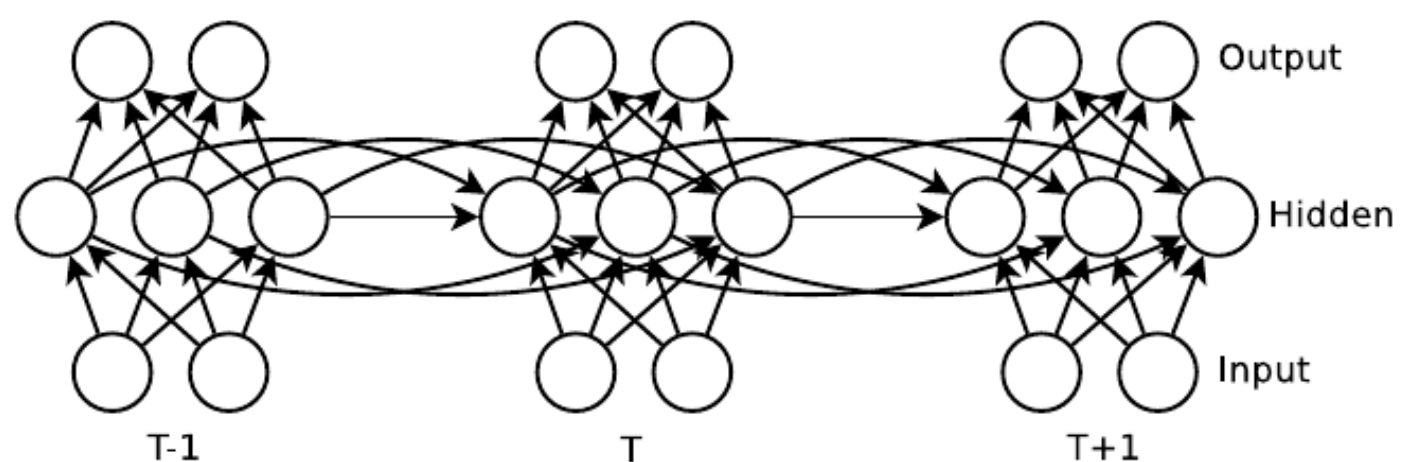

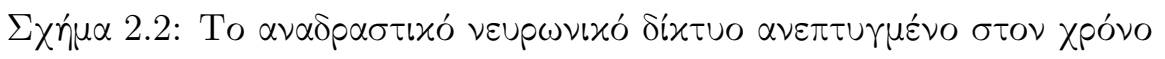

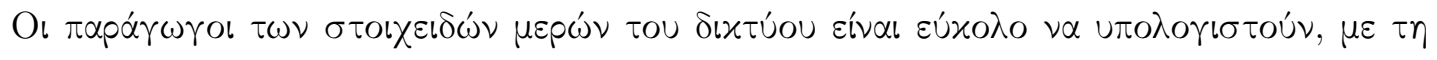

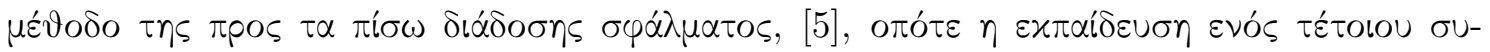

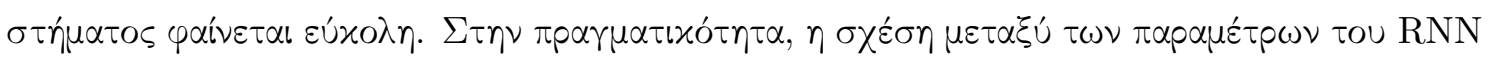

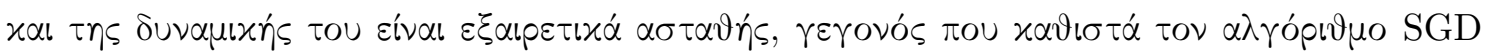

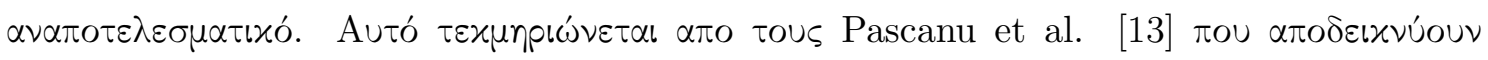

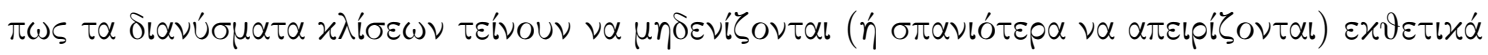

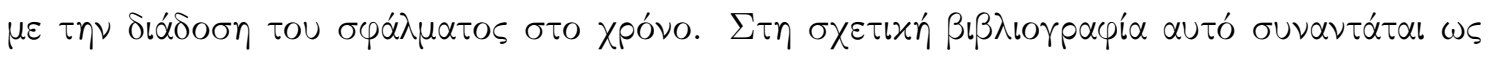

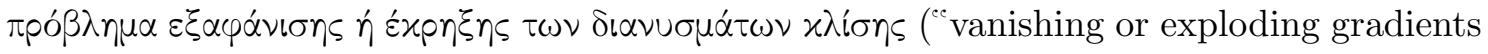




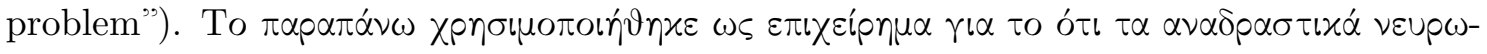

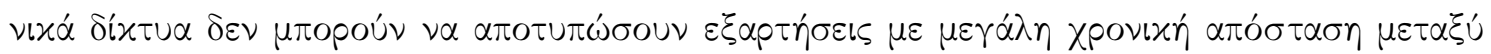

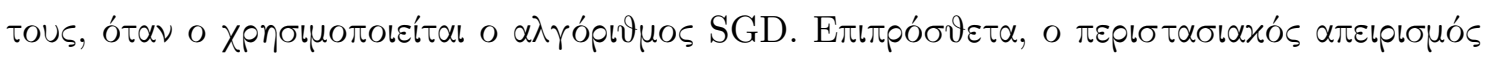

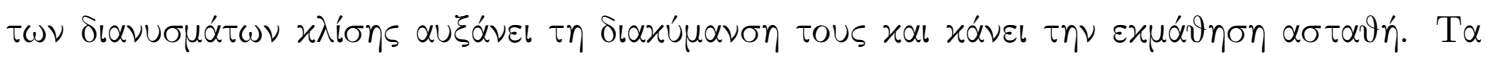

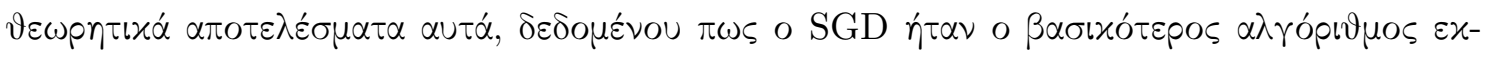

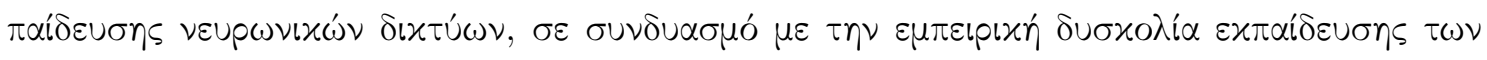

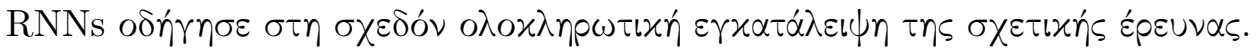

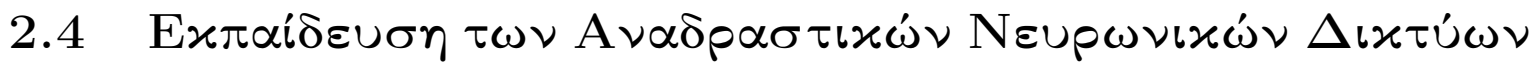

\subsubsection{Long Short-Term Memory Units}

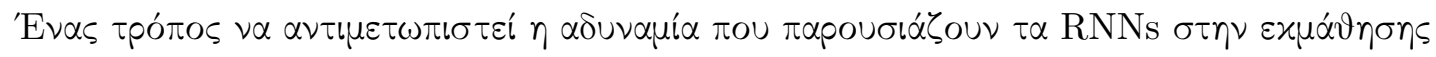

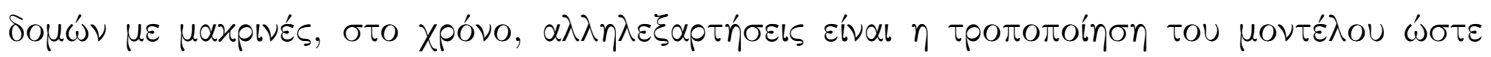

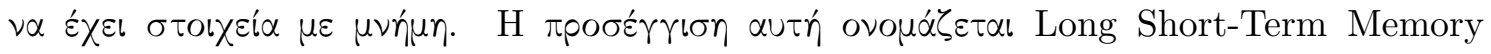

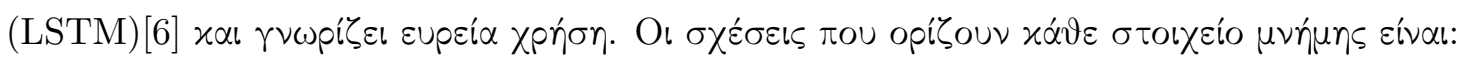

$$
\begin{aligned}
& i_{t}=\sigma\left(W_{x i} x_{t}+W_{h i} h_{t-1}+W_{c i} c_{t-1}+b_{i}\right) \\
& f_{t}=\sigma\left(W_{x f} x_{t}+W_{h f} h_{t-1}+W_{c f} c_{t-1}+b_{f}\right) \\
& c_{t}=f_{t} c_{t-1}+i_{t} \tanh \left(W_{x c} x_{t}+W_{h c} h_{t-1}+b_{c}\right) \\
& o_{t}=\sigma\left(W_{x o} x_{t}+W_{h o} h_{t-1}+W_{c o} c_{t}+b_{o}\right) \\
& h_{t}=o_{t} \tanh c_{t}
\end{aligned}
$$

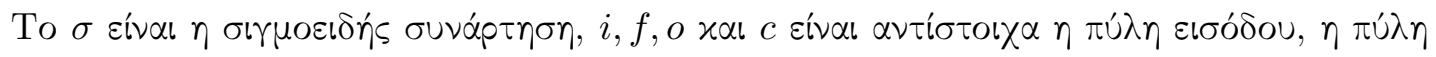

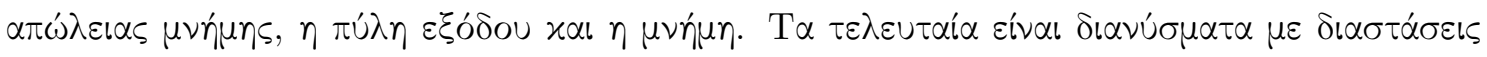

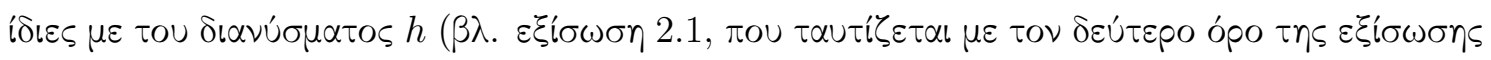

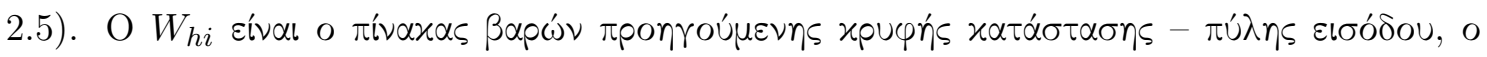

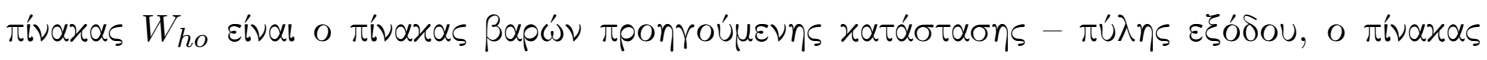

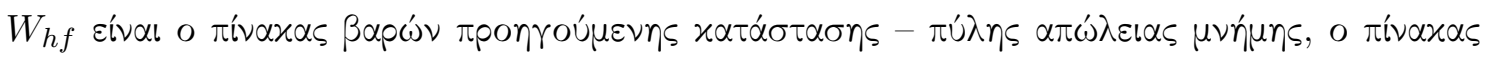

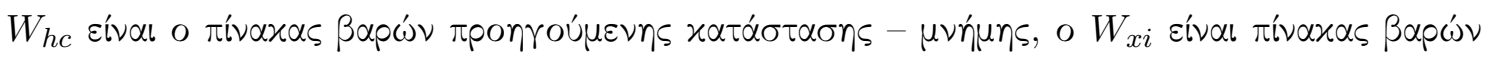

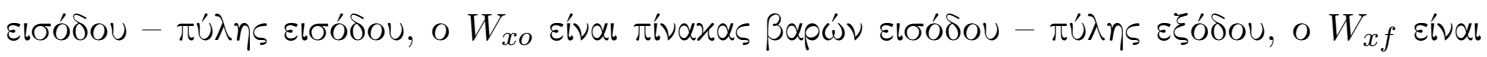

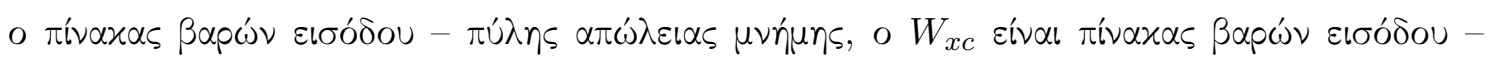

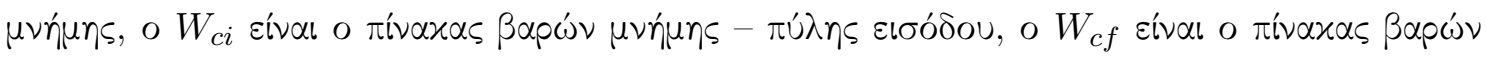

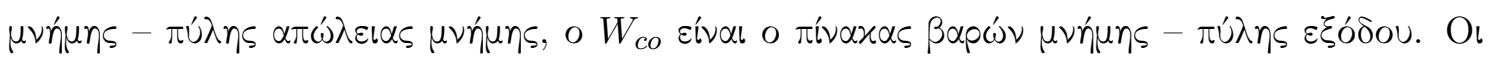

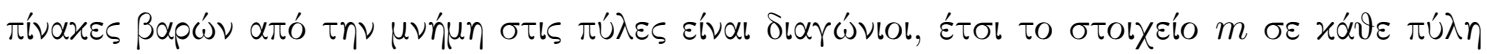

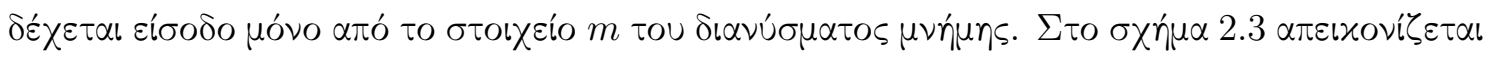

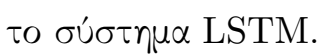

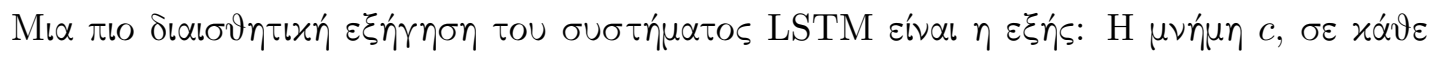

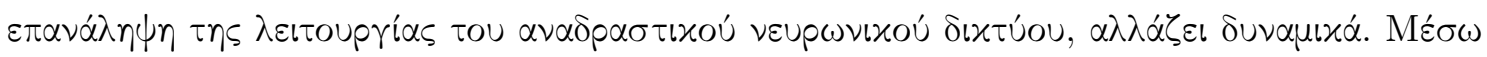




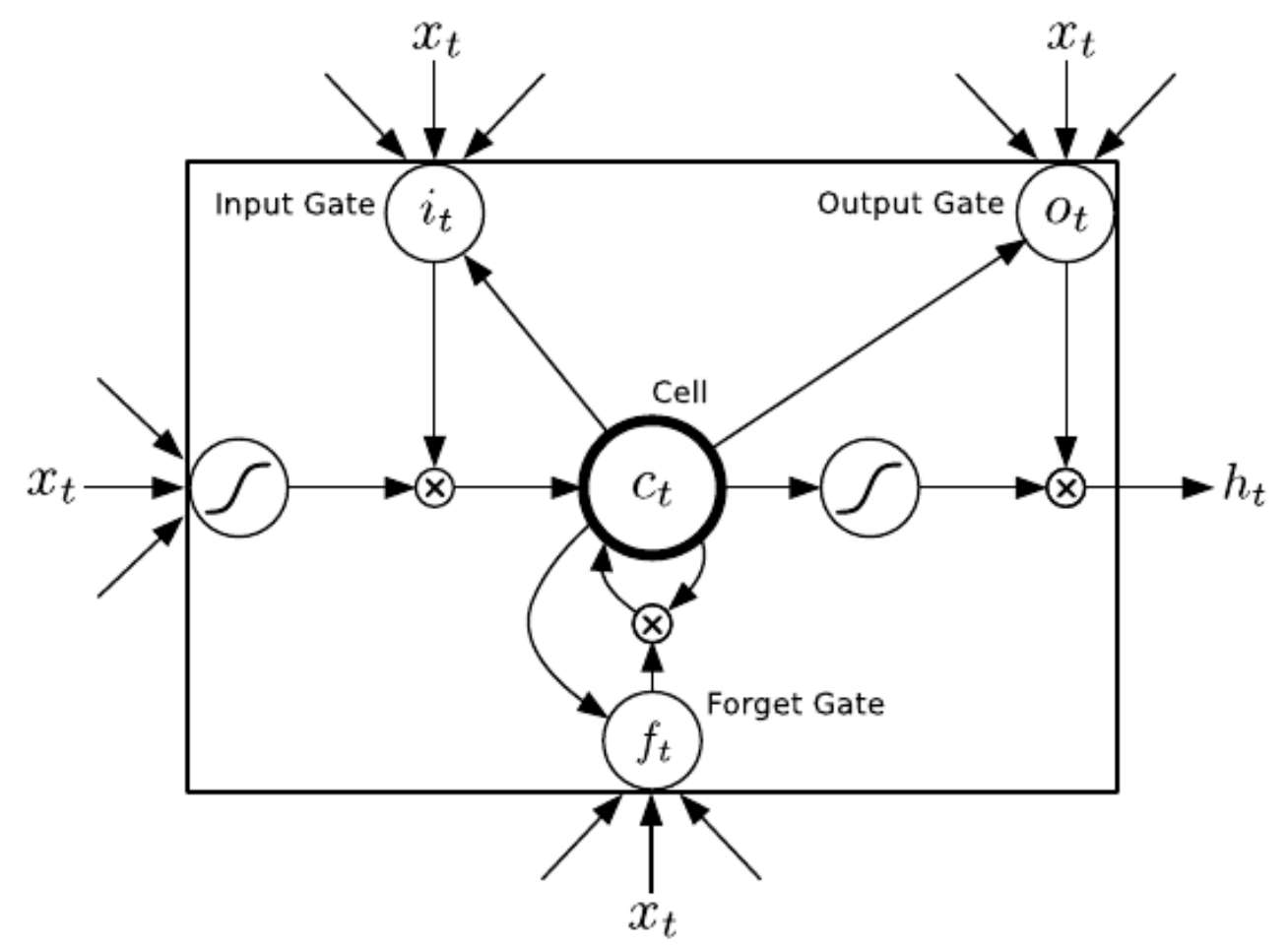

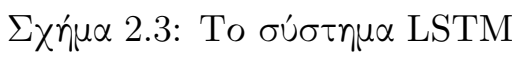

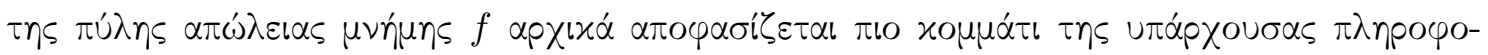

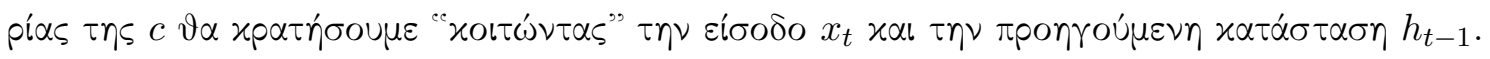

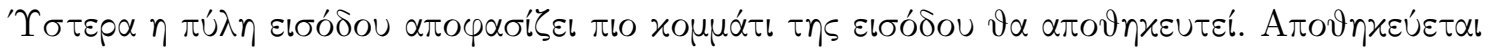

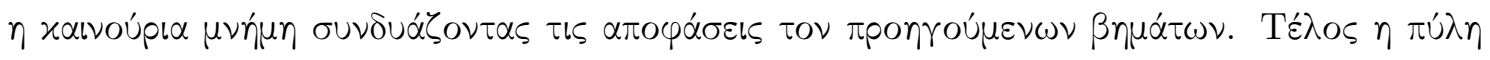

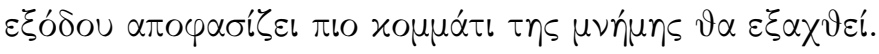

\subsubsection{Truncated Backpropagaion Through Time}

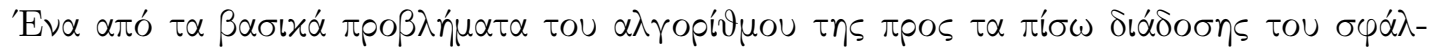

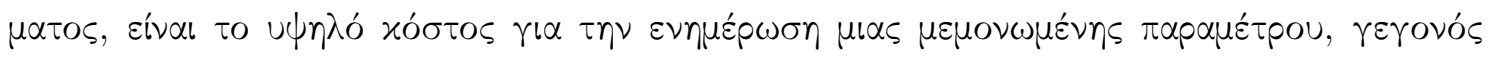

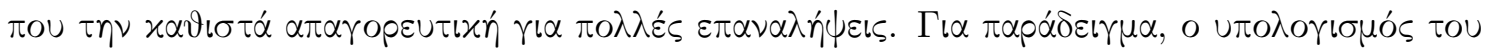

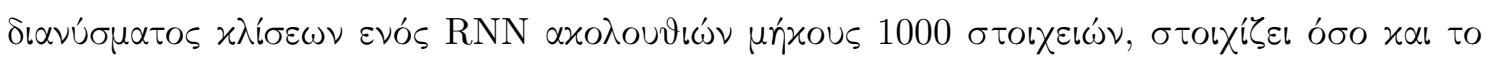

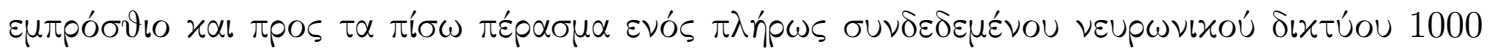

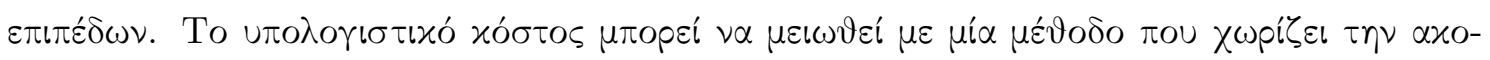

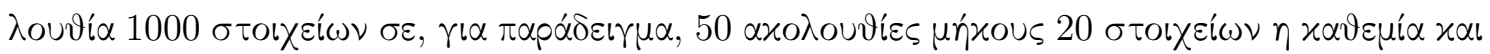

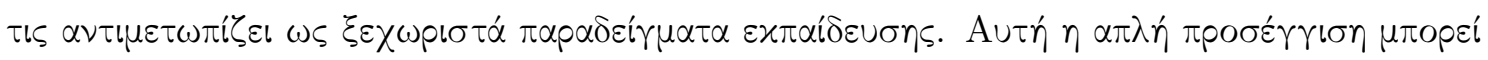

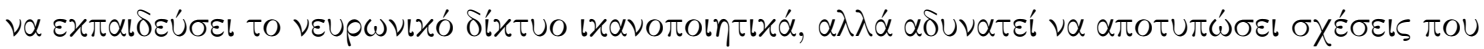

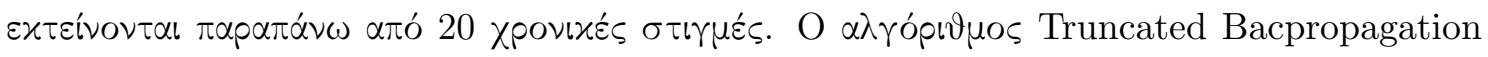

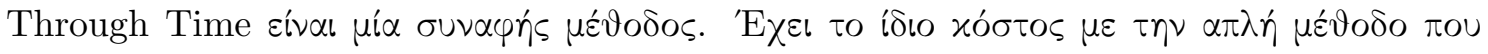




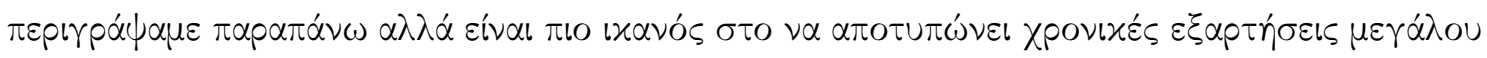

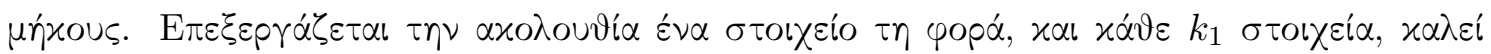

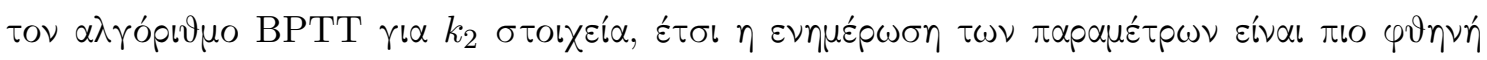

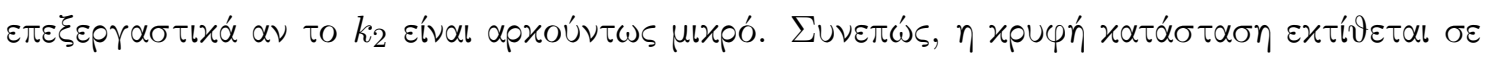

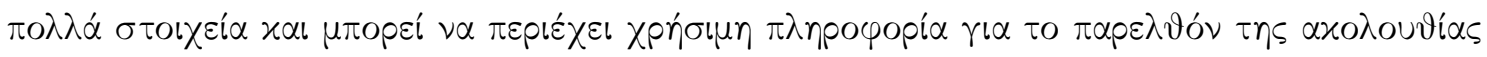

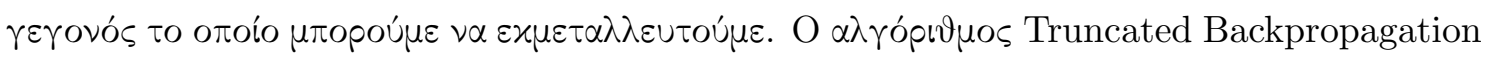
Through Time:

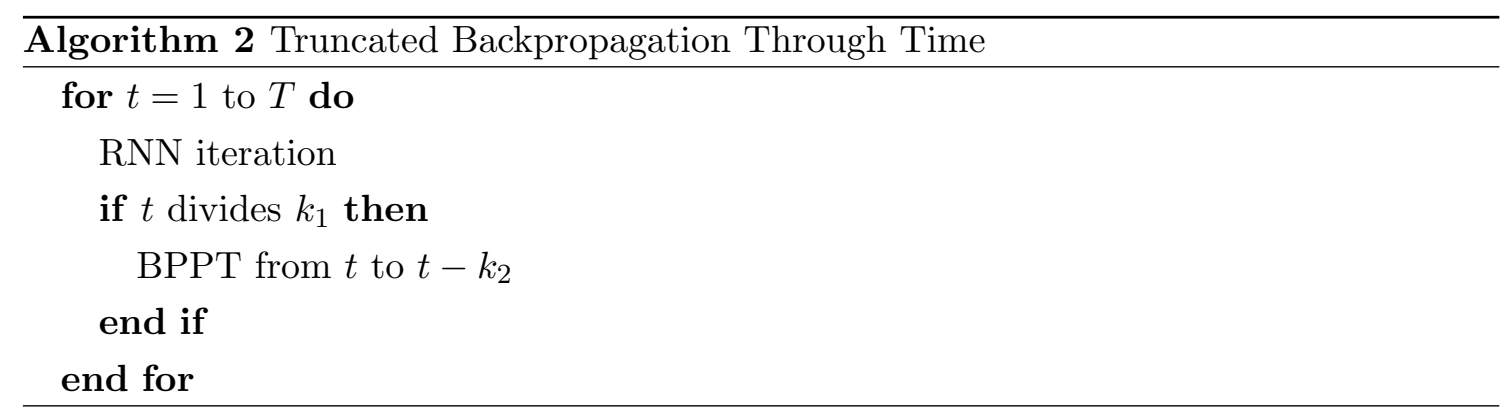

\subsubsection{Dropout}

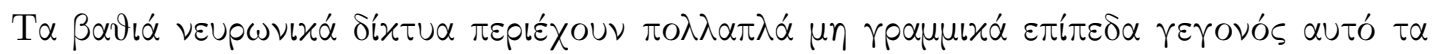

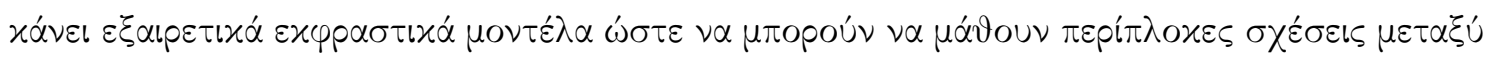

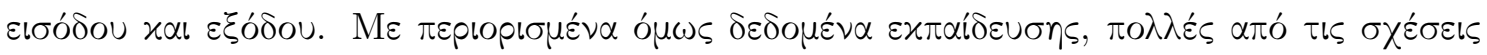

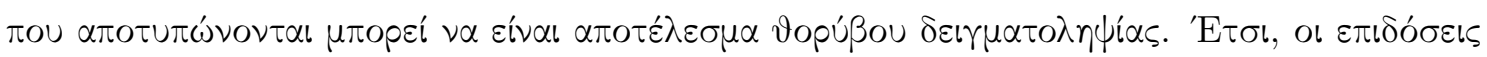

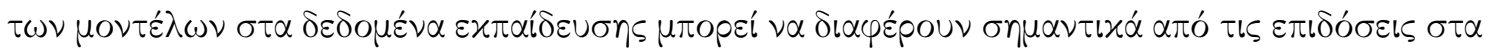

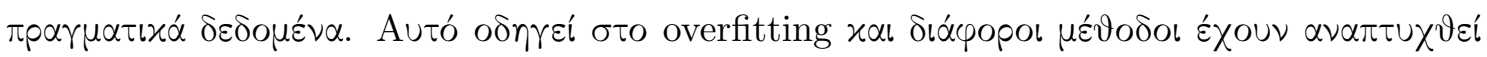

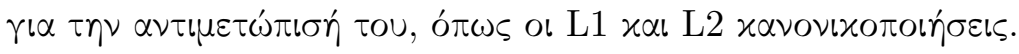

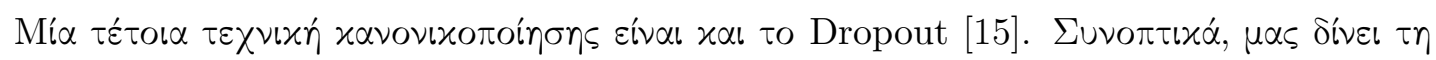

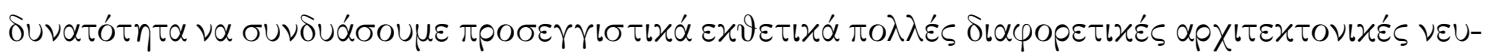

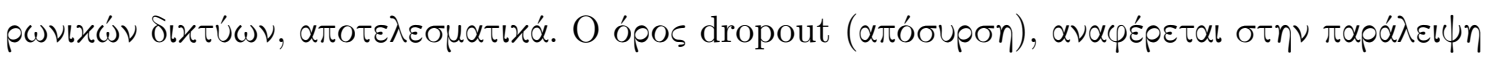

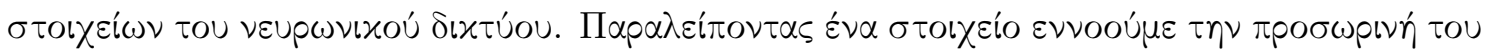

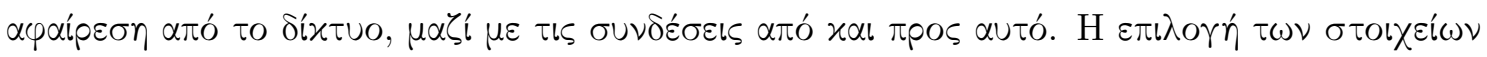

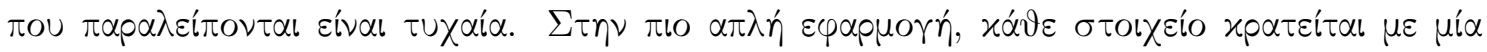

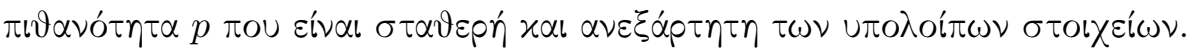

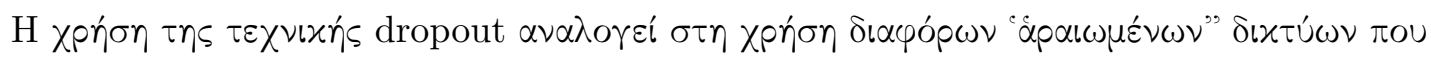

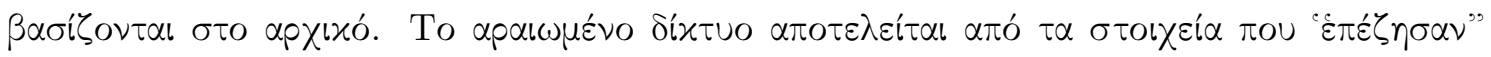

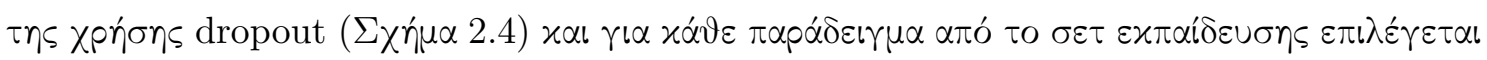

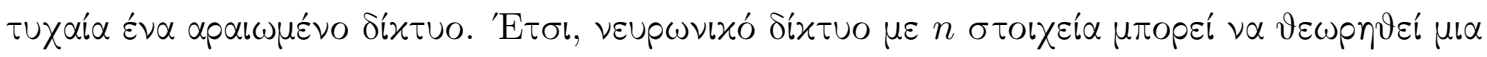

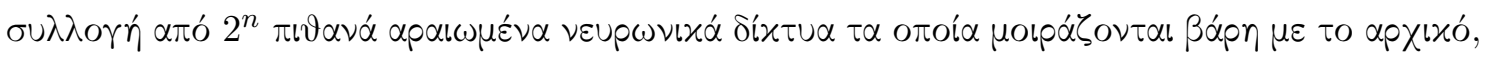

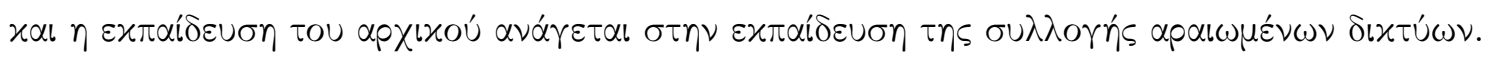

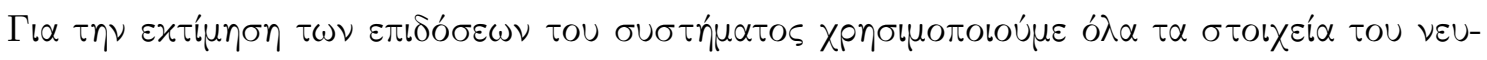

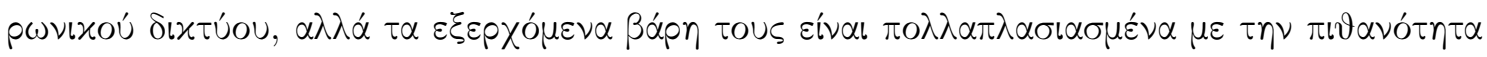
$p$. 

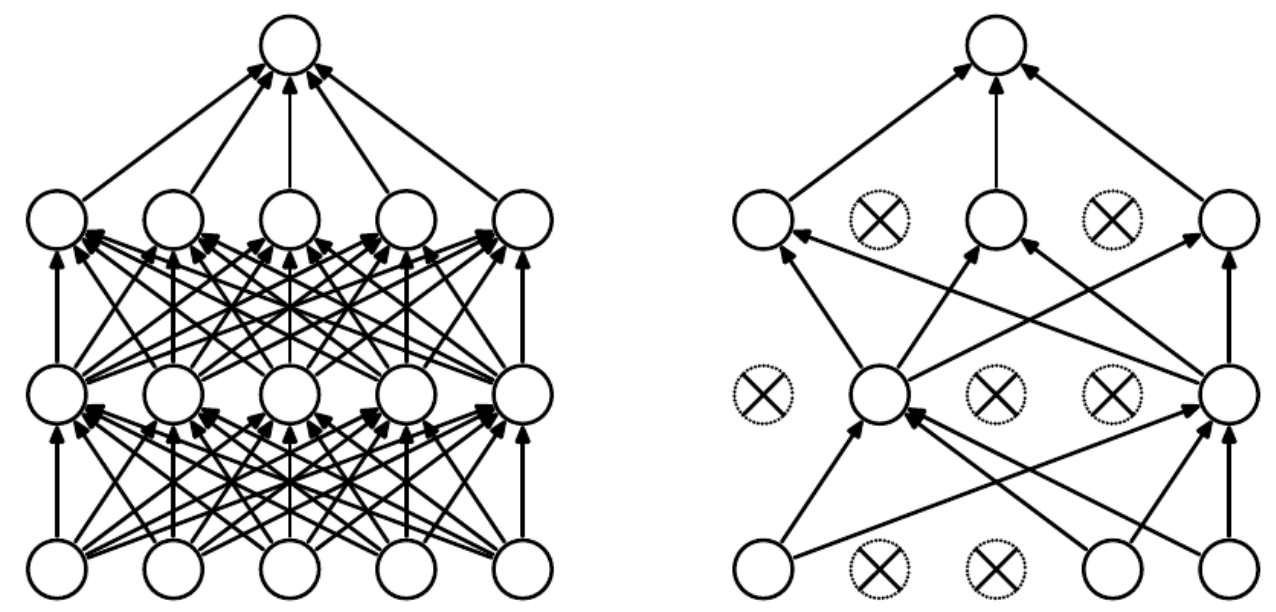

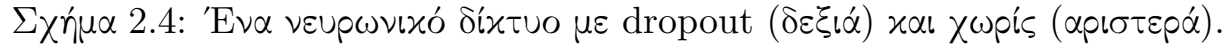




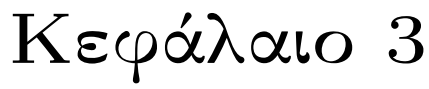

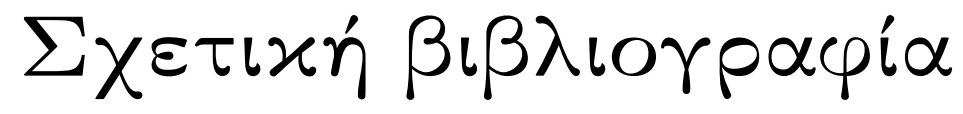

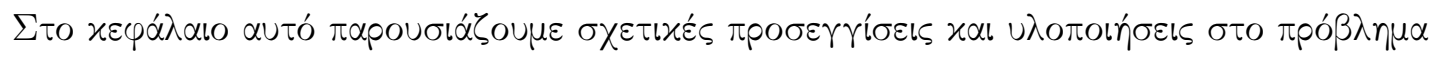

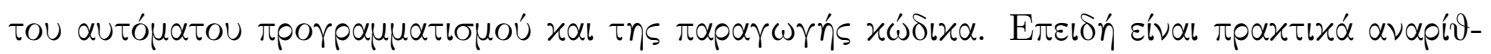

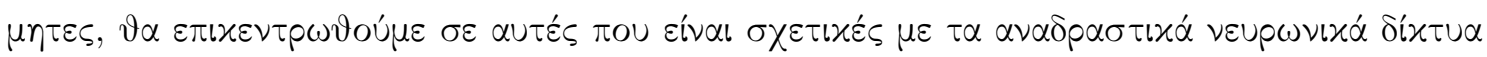

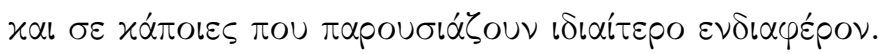

\subsection{Generating Sequence with Recurrent Neural Networks}

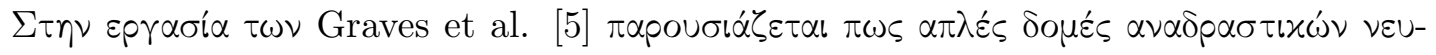

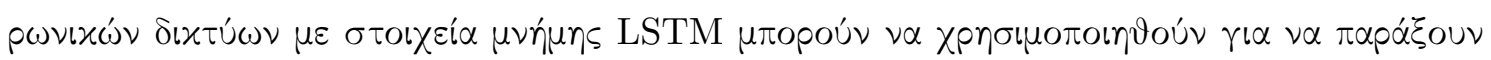

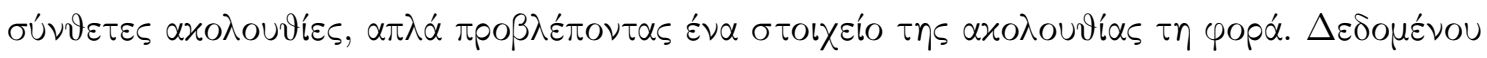

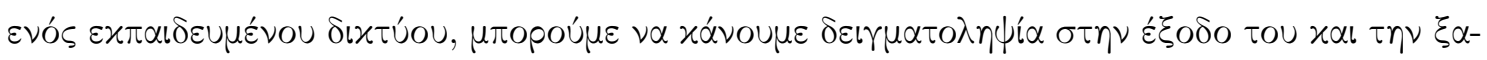

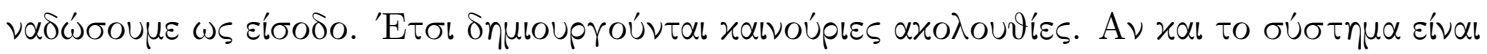

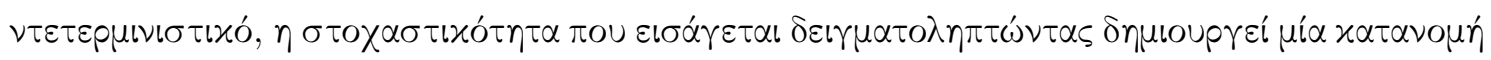

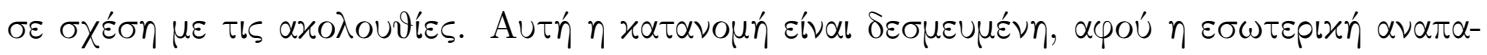

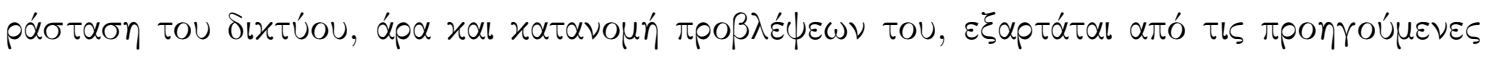

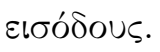

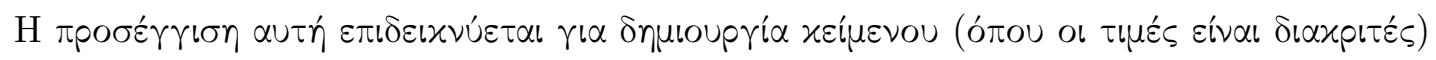

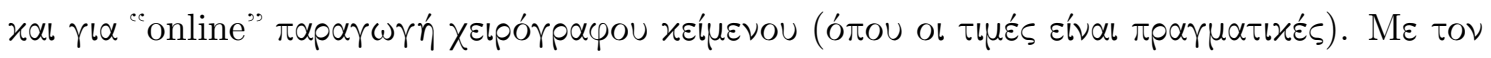

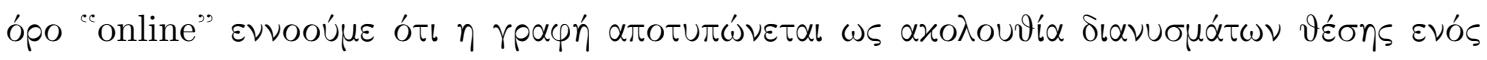

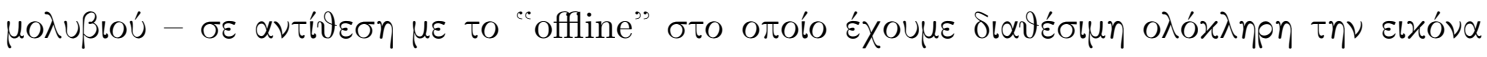

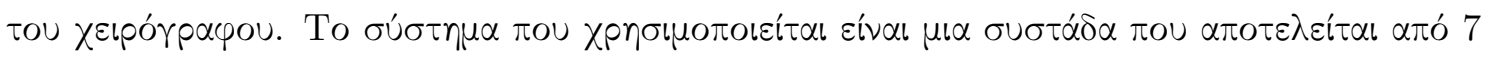

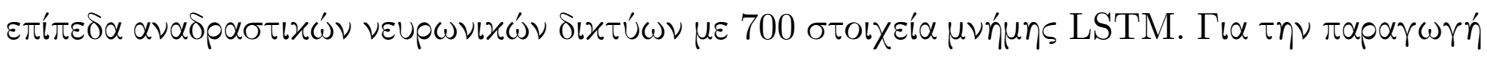

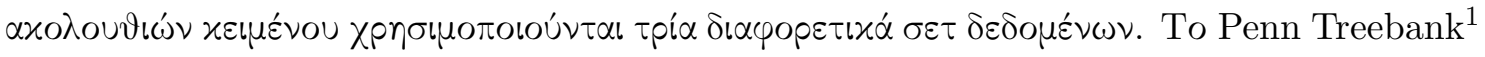

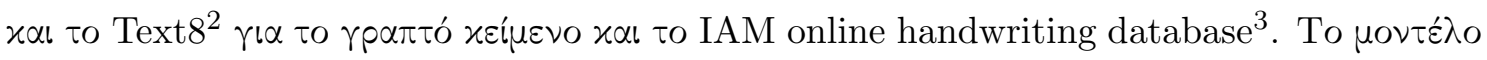

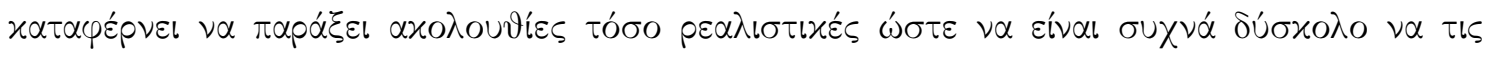

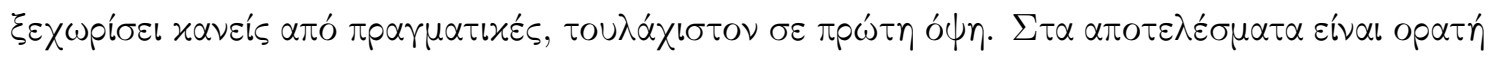

\footnotetext{
${ }^{1}$ https://catalog.1dc.upenn.edu/ldc99t42

${ }^{2}$ http://mattmahoney.net/dc/textdata

${ }^{3}$ http://www.fki.inf. unibe.ch/databases/iam-on-line-handwriting-database
} 


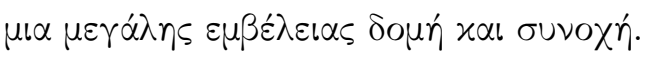

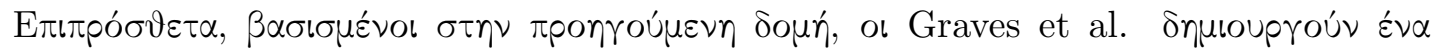

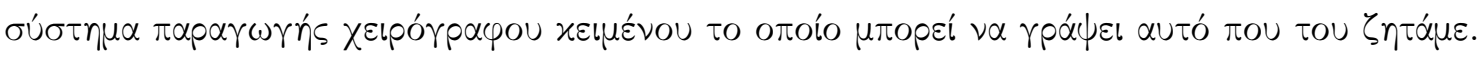

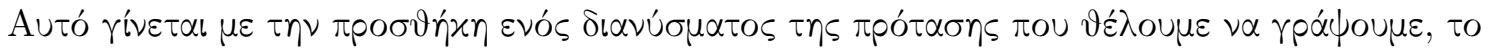

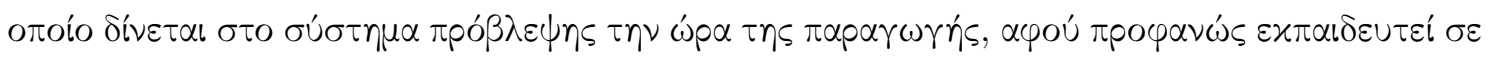

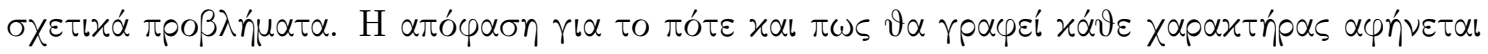

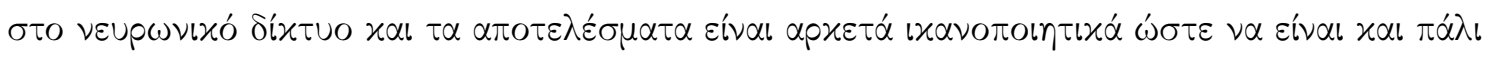

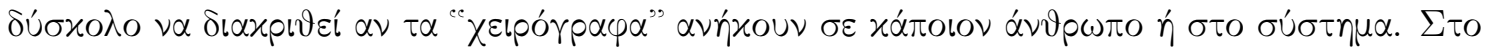

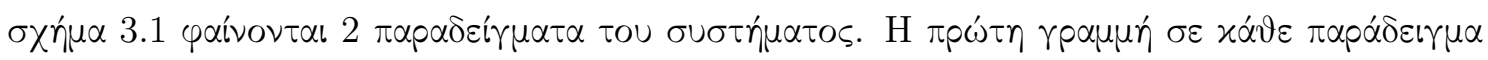

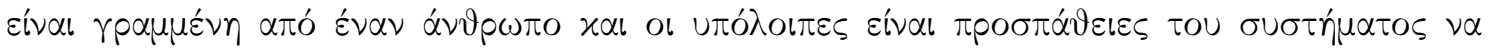

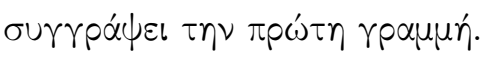

\subsection{Inferring Algorithmic Patterns with Stack-Augmented Recurrent Nets}

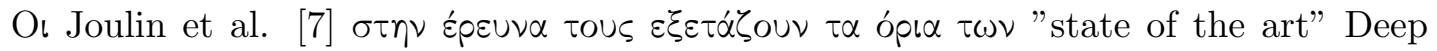

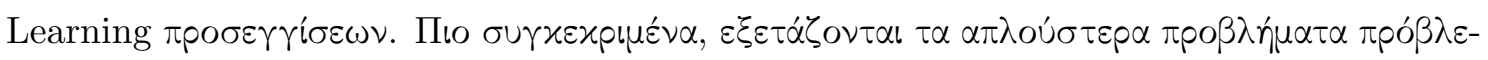

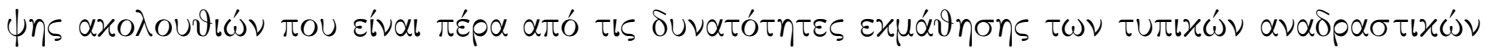

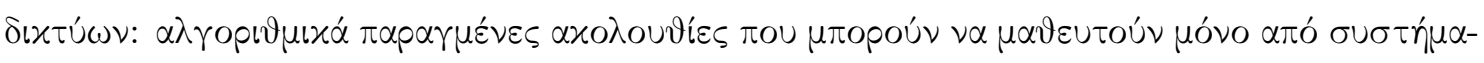

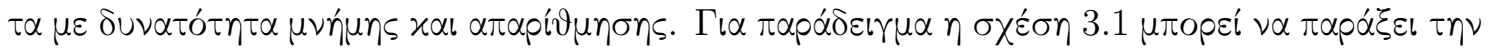

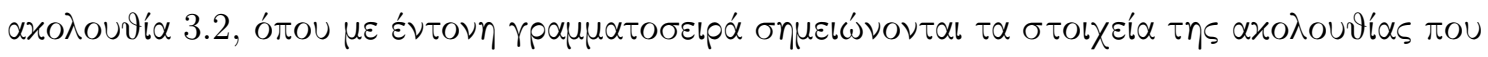

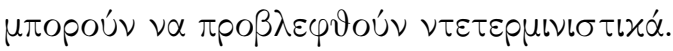

$$
a^{n} b^{n}, n>0
$$

aabbaaabbbabaaaaabbbbb

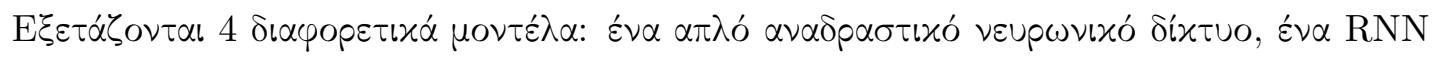

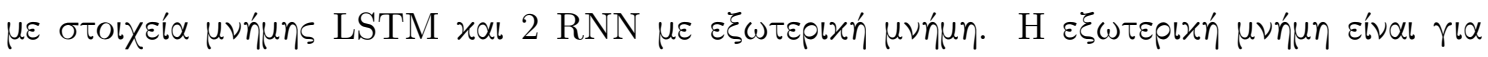

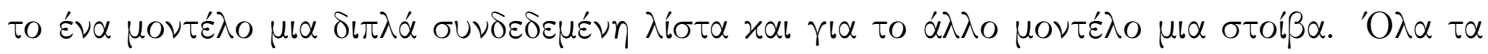

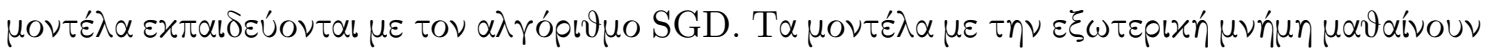

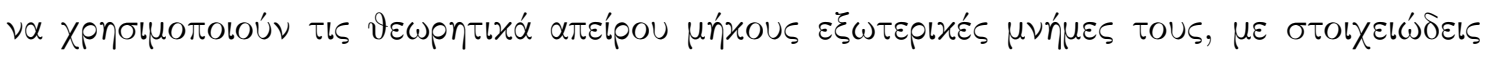

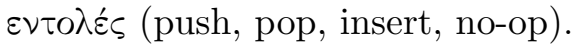

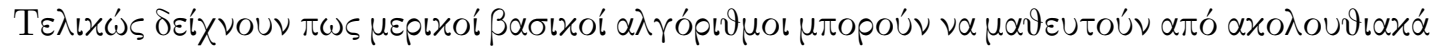

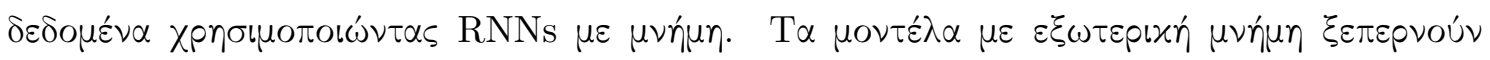

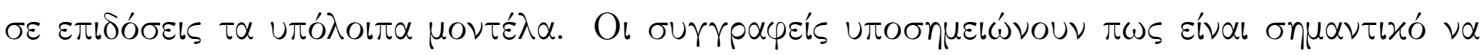

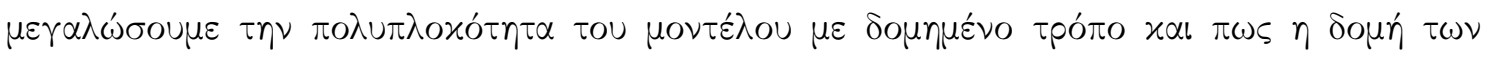

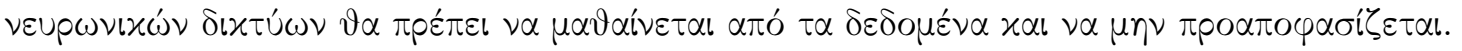


3.2 Inferring Algorithmic Patterns with Stack-Augmented Recurrent Nets

27

from his travels it might have been from his travels it might have been from his travels it might have been from his travel itsmightmare born flower see trowels it might have ben from hes travels - it might have been

mole of national temperament move of national temperament more of national temperament use of nations temperament mace of nation id temperament more of national temperament

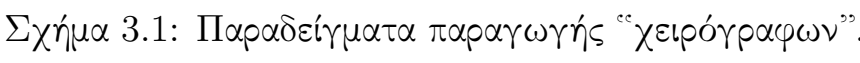




\subsection{A Synthetic Neural Model for General Purpose Code Generation}

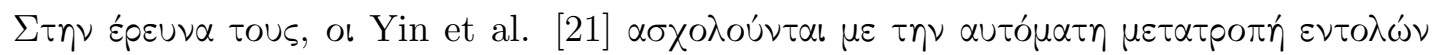

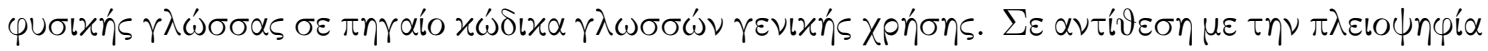

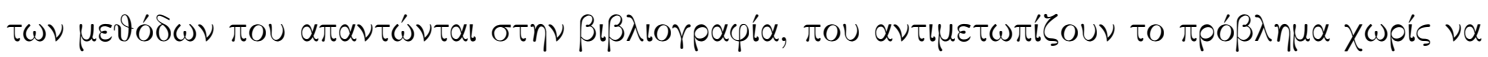

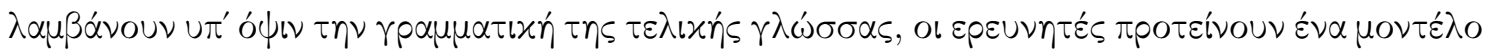

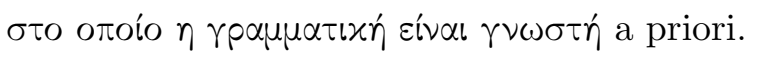

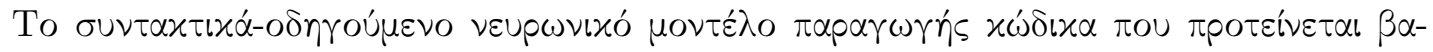

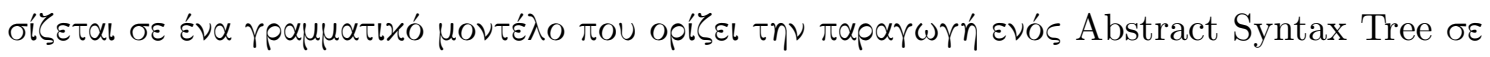

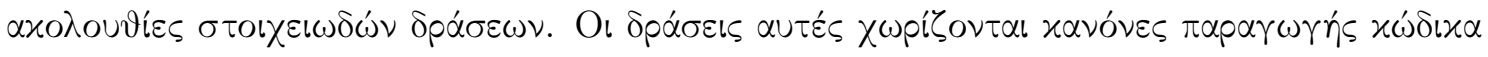

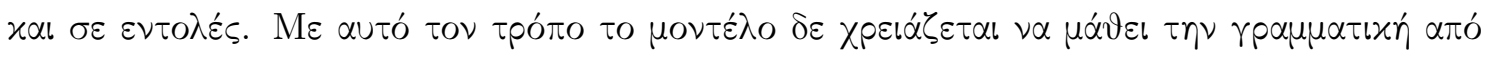

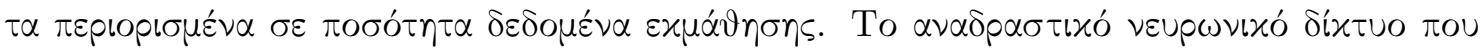

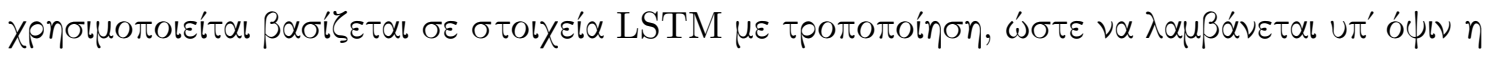

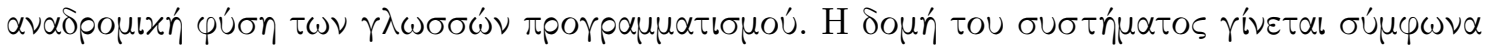

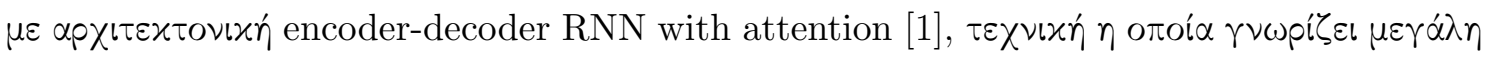

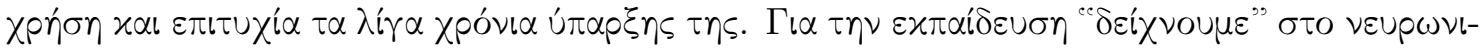

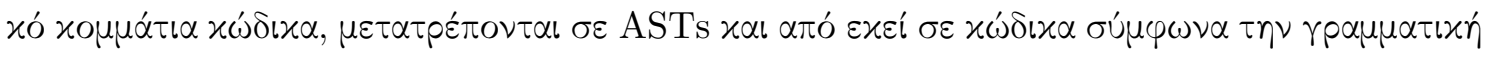

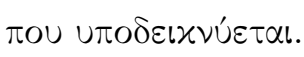

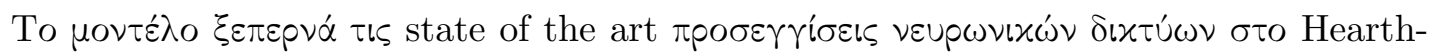

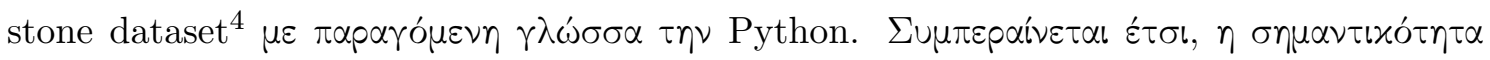

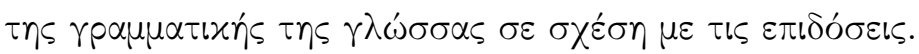

\subsection{End-to-End Memory Networks}

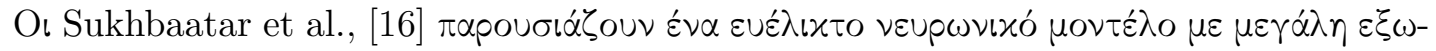

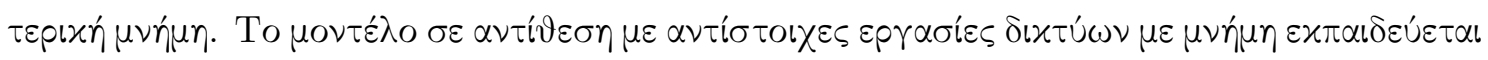

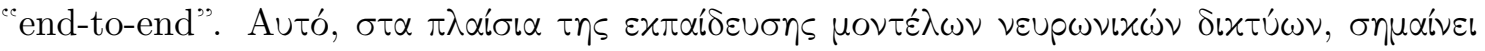

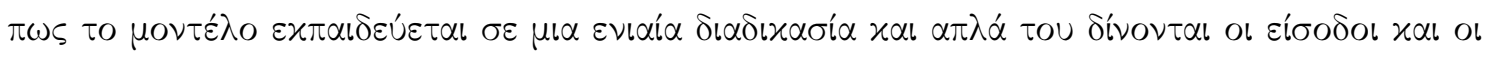

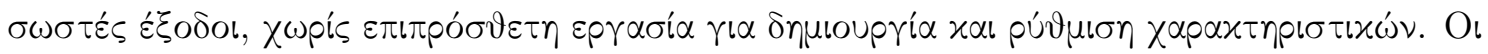

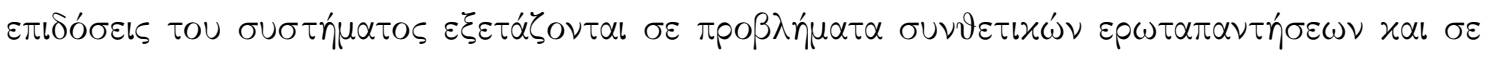

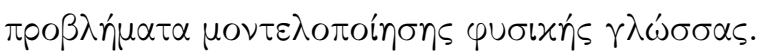

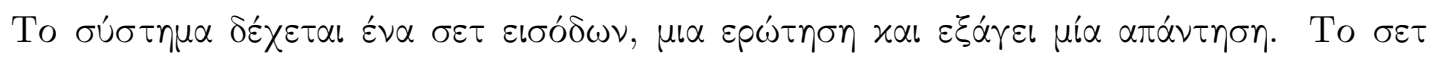

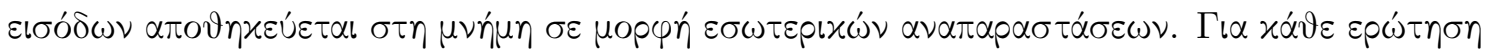

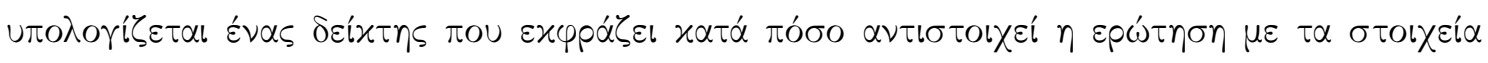

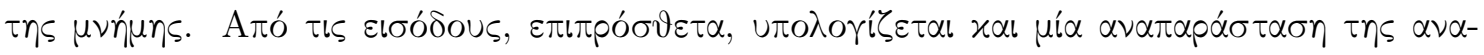

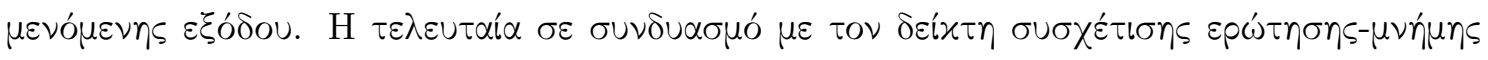

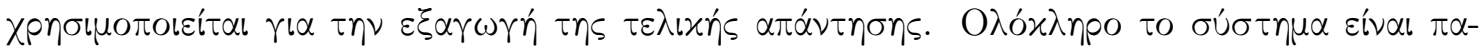

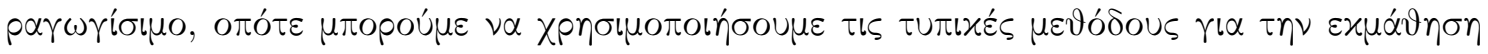

\footnotetext{
${ }^{4}$ https://github.com/deepmind/card2 code
} 
ToU.

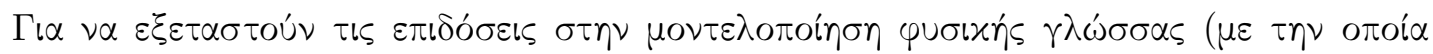

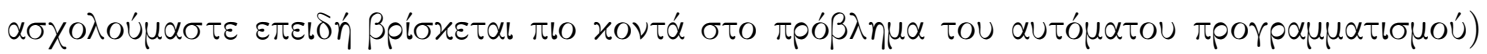

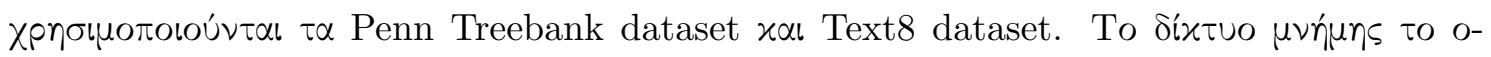

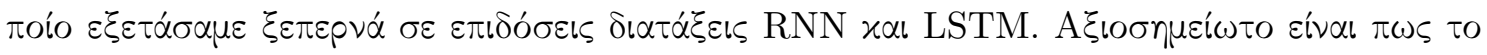

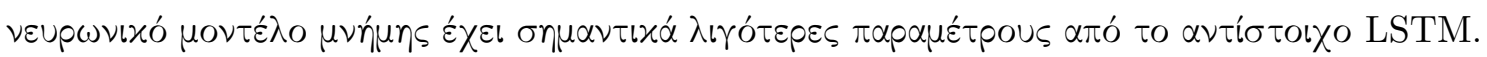

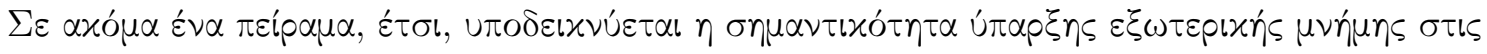

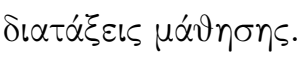

\subsection{Neuro Symbolic Program Synthesis}

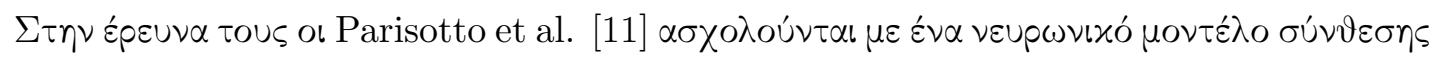

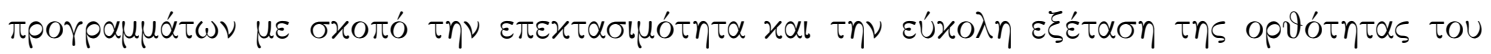

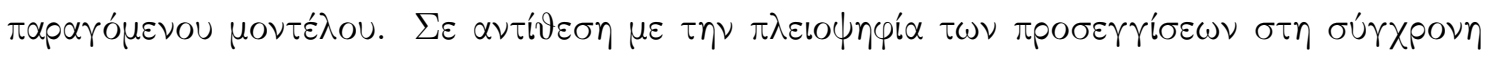

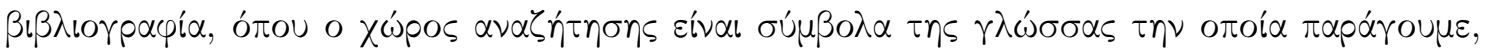

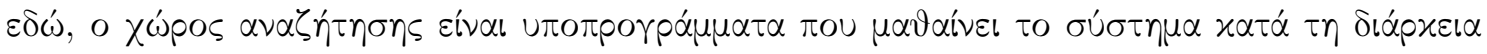

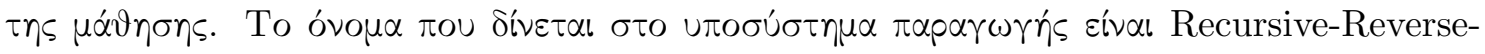
Recursive Neural Network (R3NN).

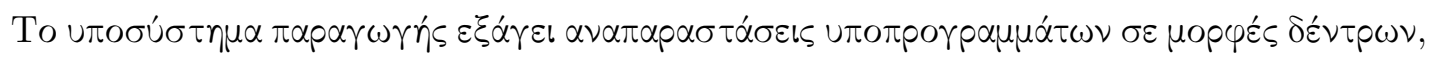

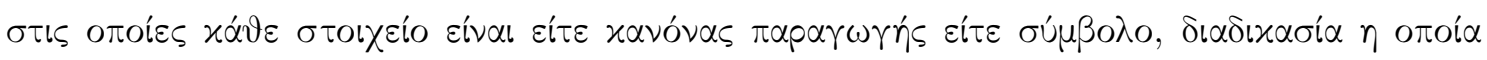

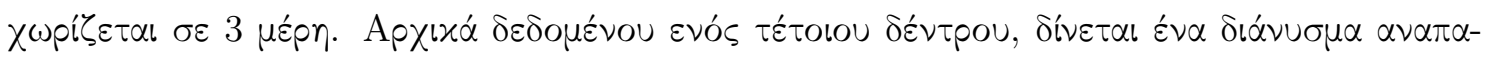

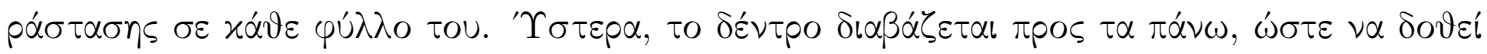

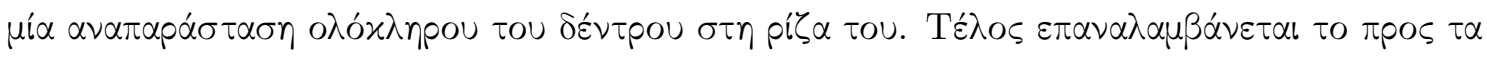

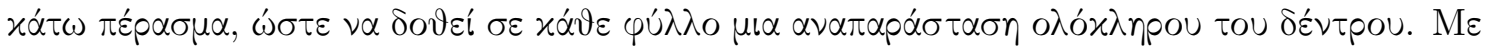

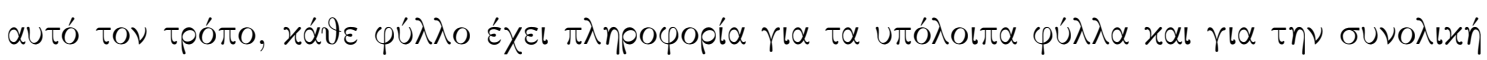

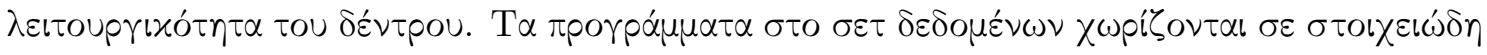

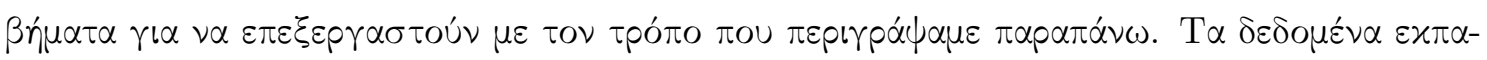

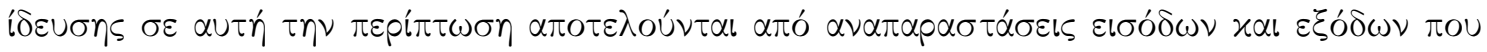

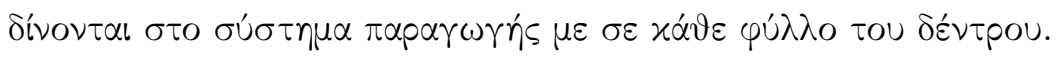

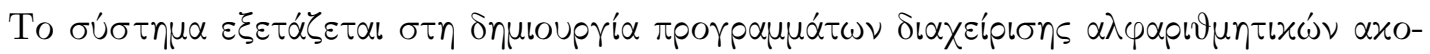

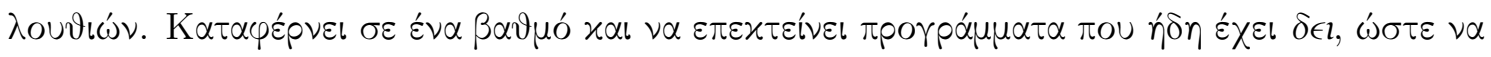

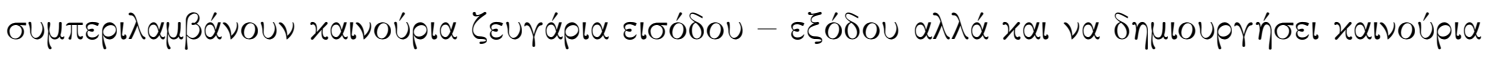

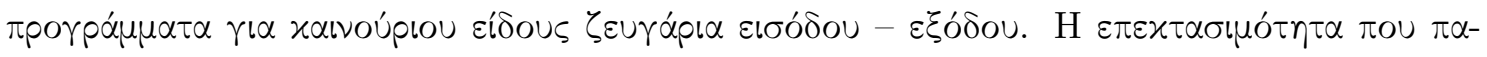

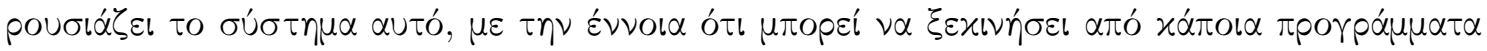

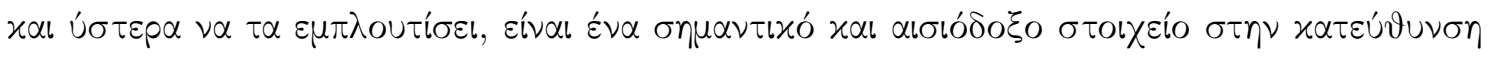

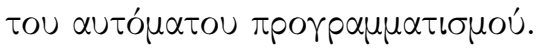





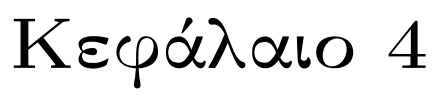

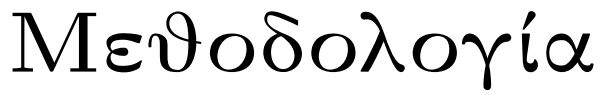

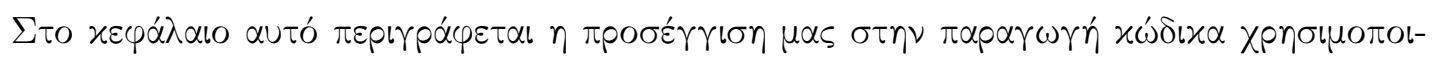

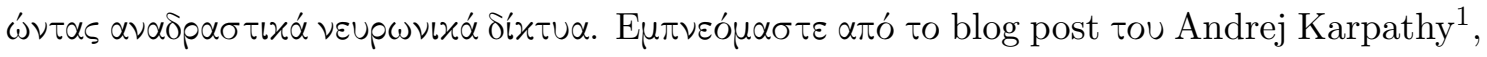

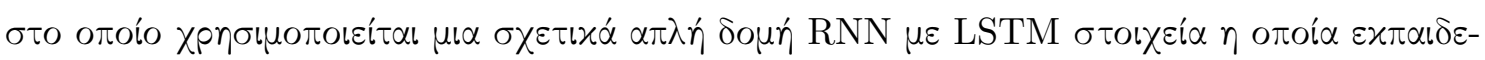

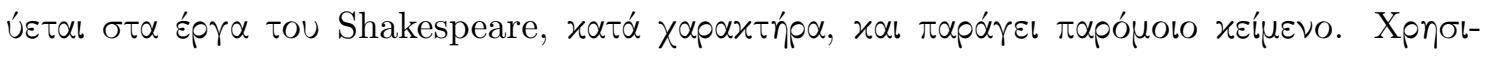

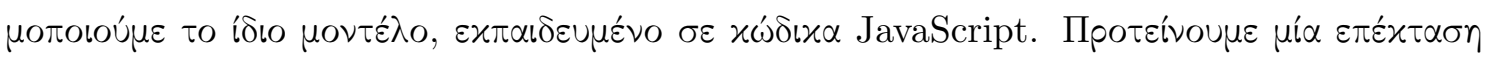

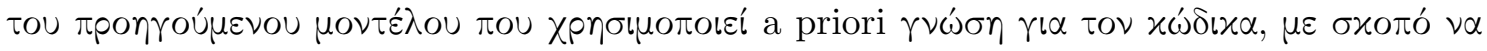

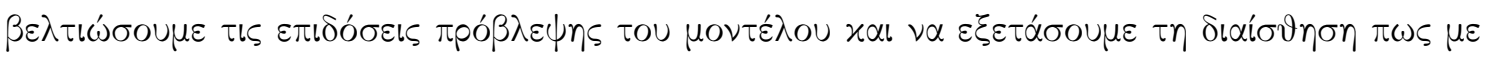

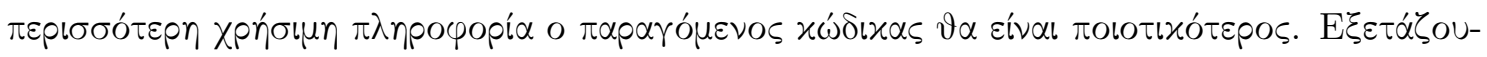

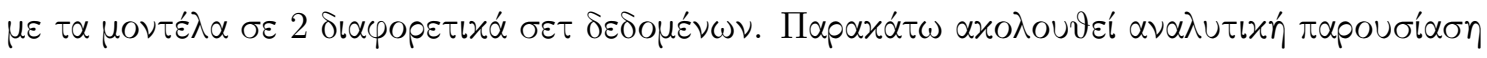

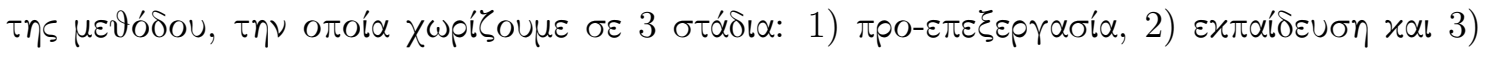

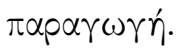

\subsection{T $\propto \mu о \nu \tau \hat{\varepsilon} \lambda \alpha$}

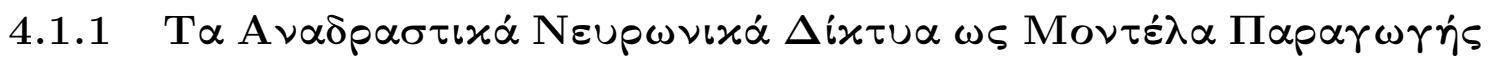

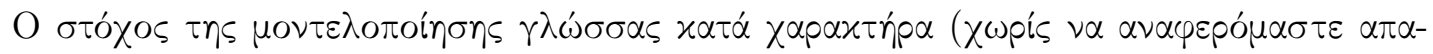

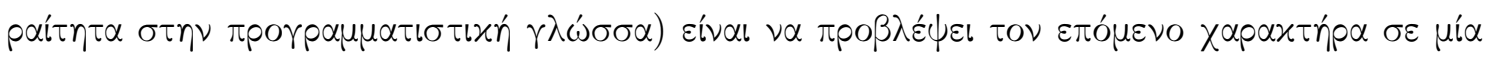

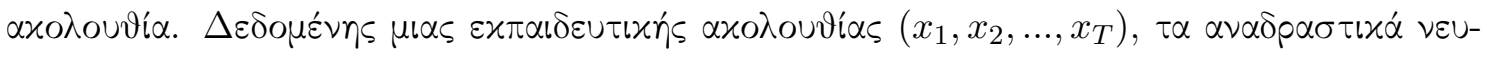

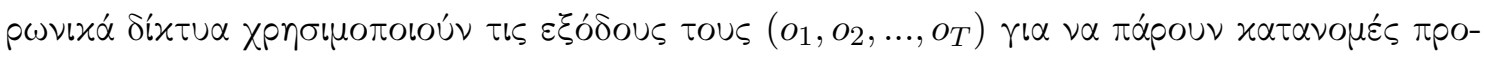

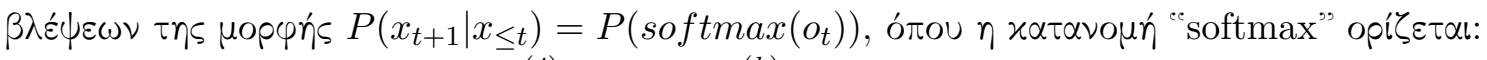

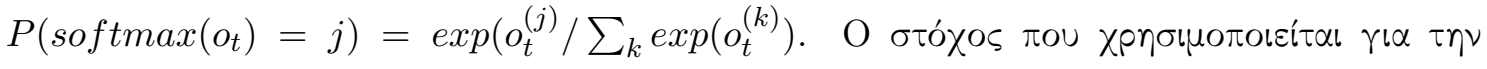

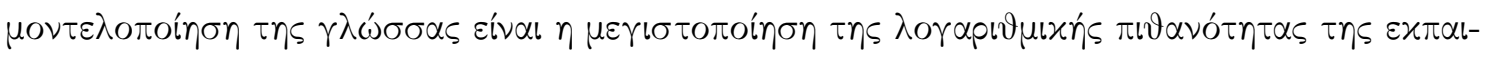

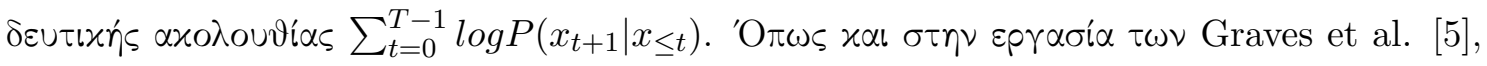

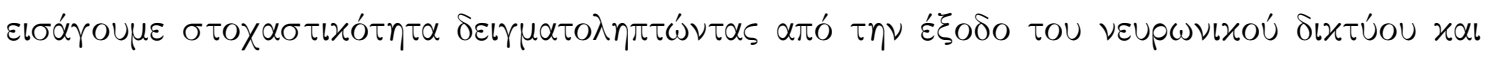

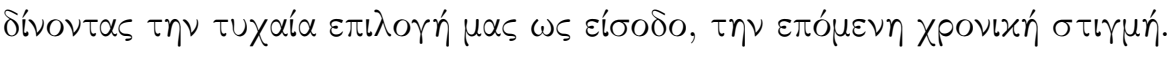

\footnotetext{
${ }^{1}$ http://karpathy.github.io/2015/05/21/rnn-effectiveness/
} 


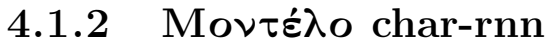

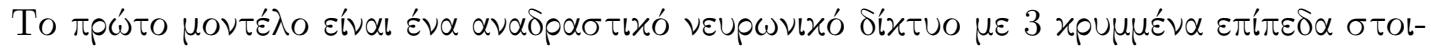

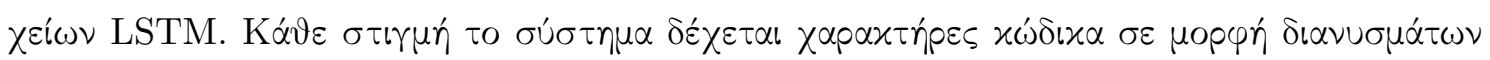

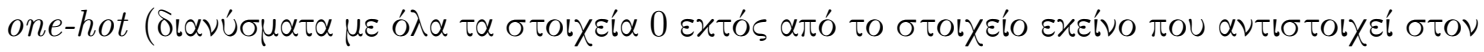

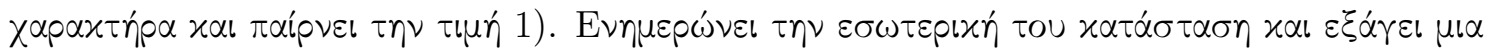

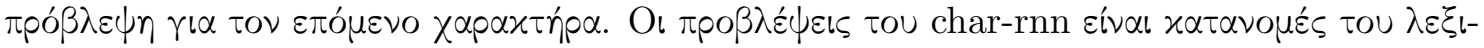

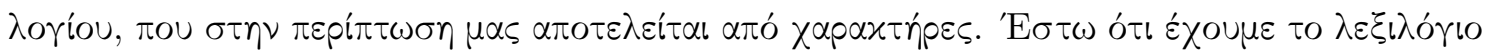

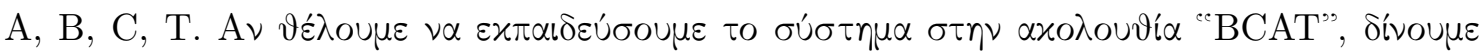

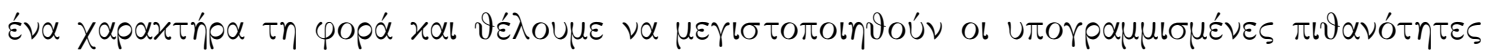

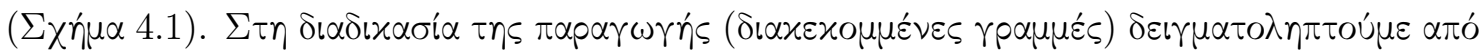

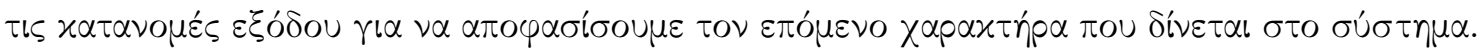

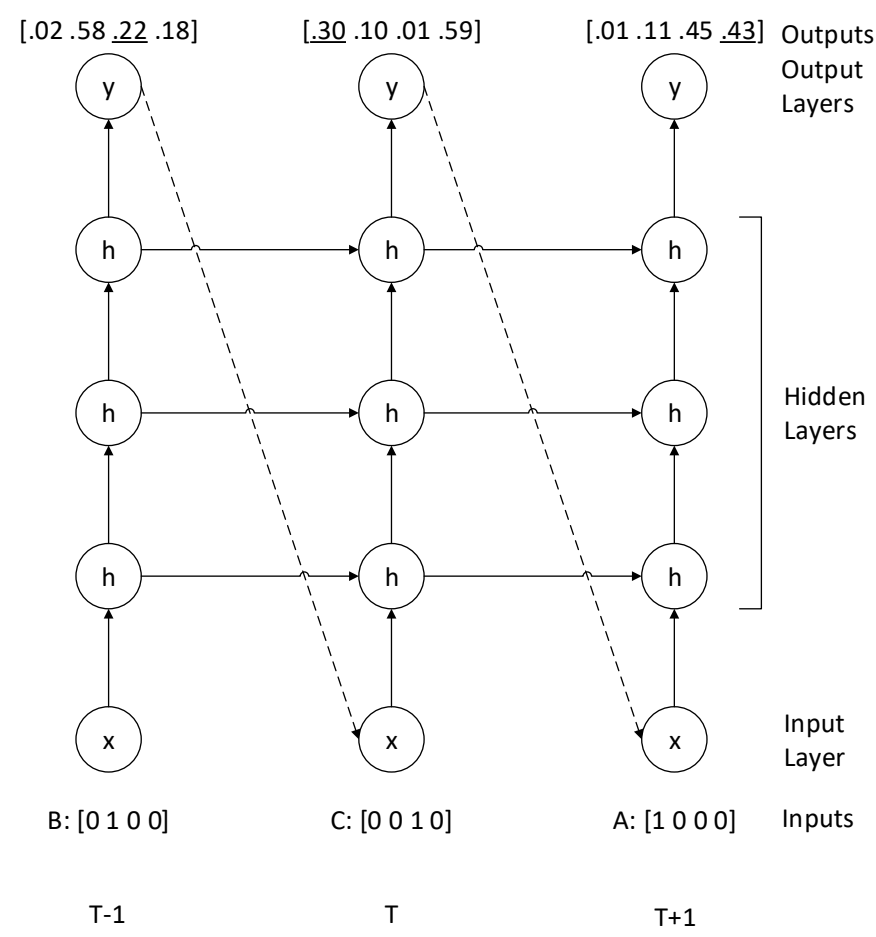

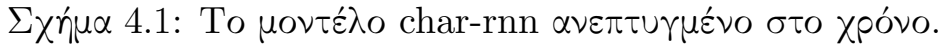

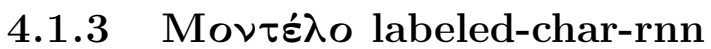

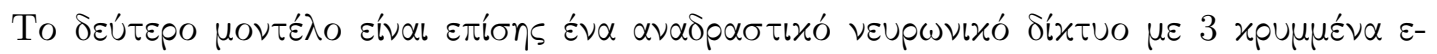

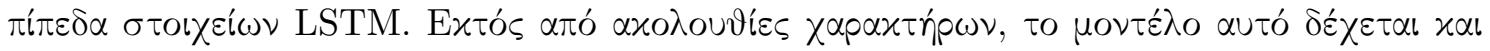

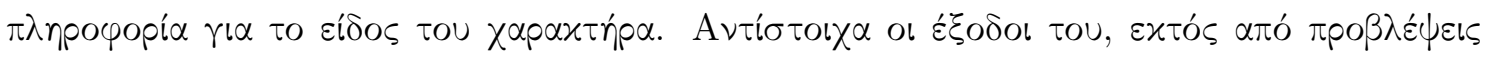

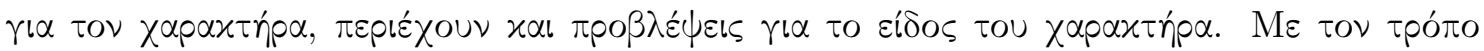

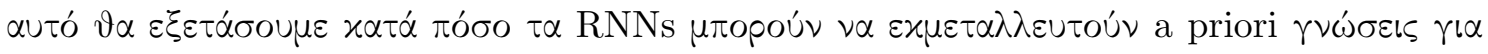

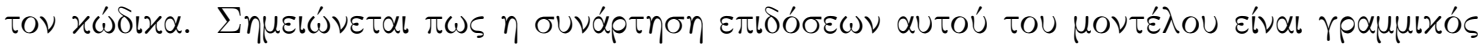




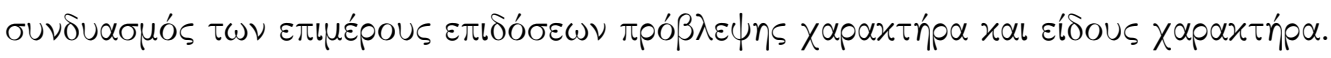

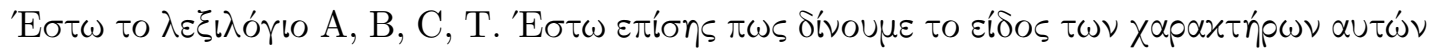

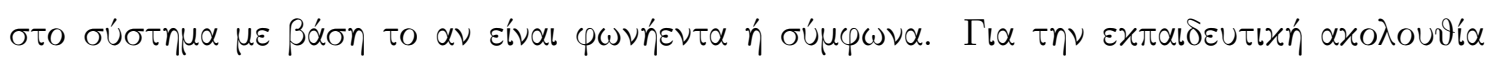

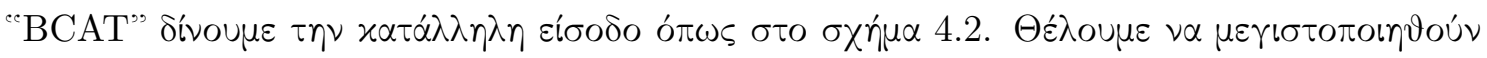

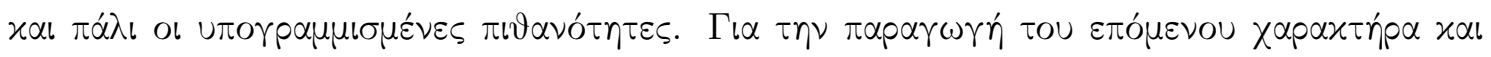

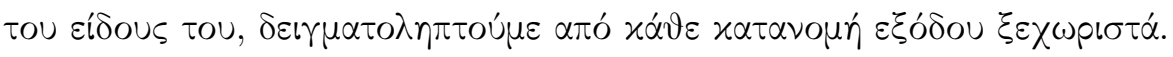

[.02.58.22.18], [.7.3] [..30.10 .01 .59], [.6.4] [.01.11.45.43], [.8.2] Outputs

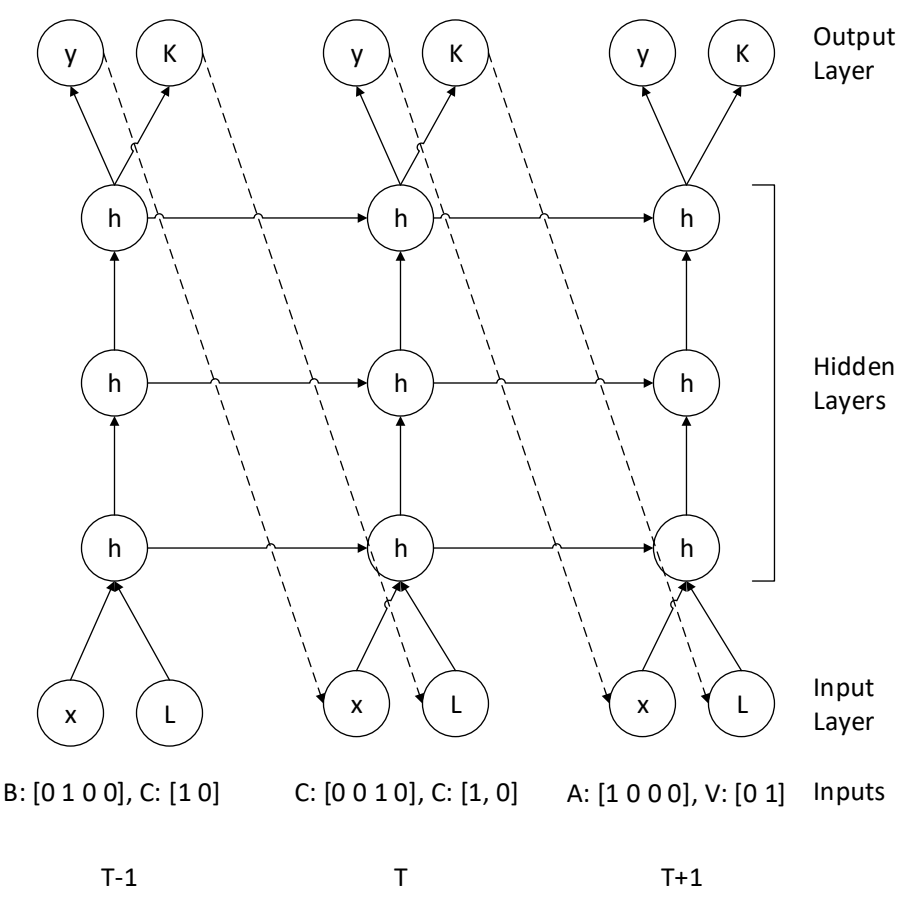

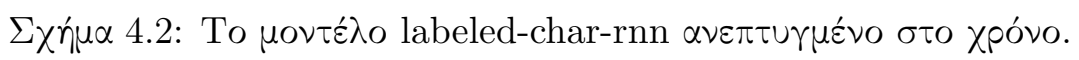

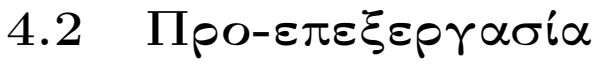

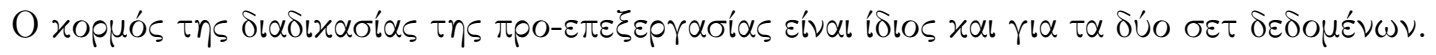

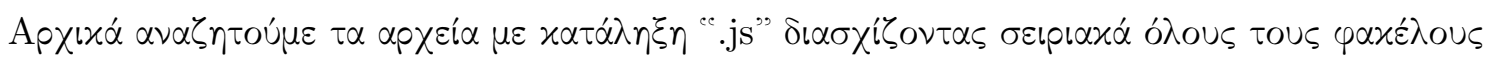

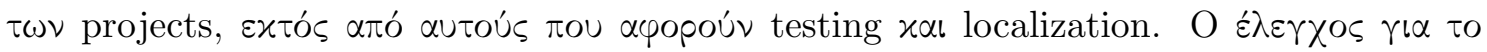

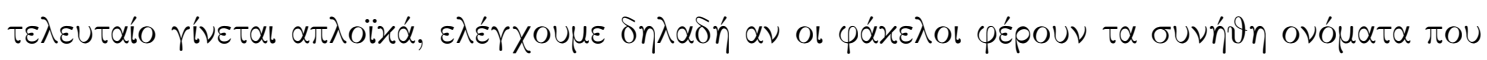

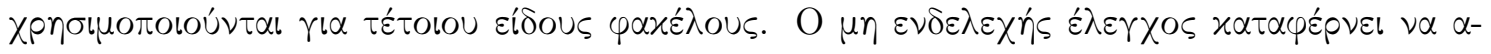

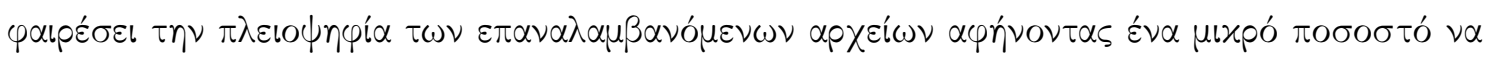

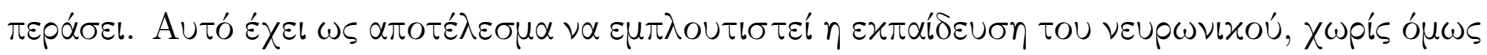

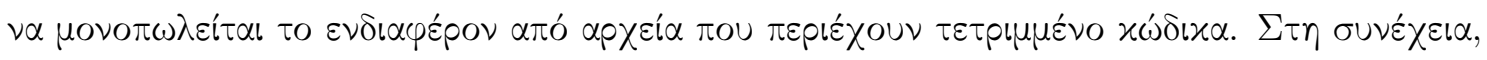

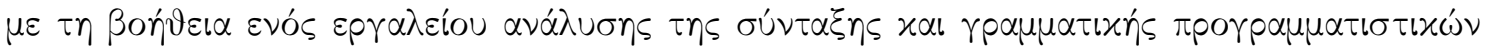




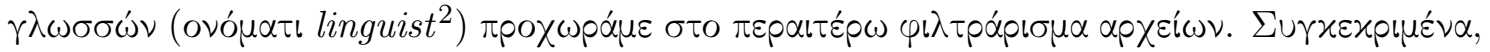

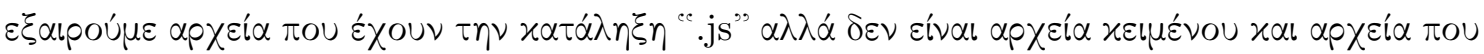

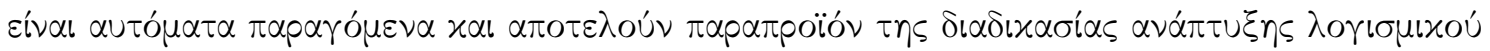
$\sigma \varepsilon$ JavaScript.

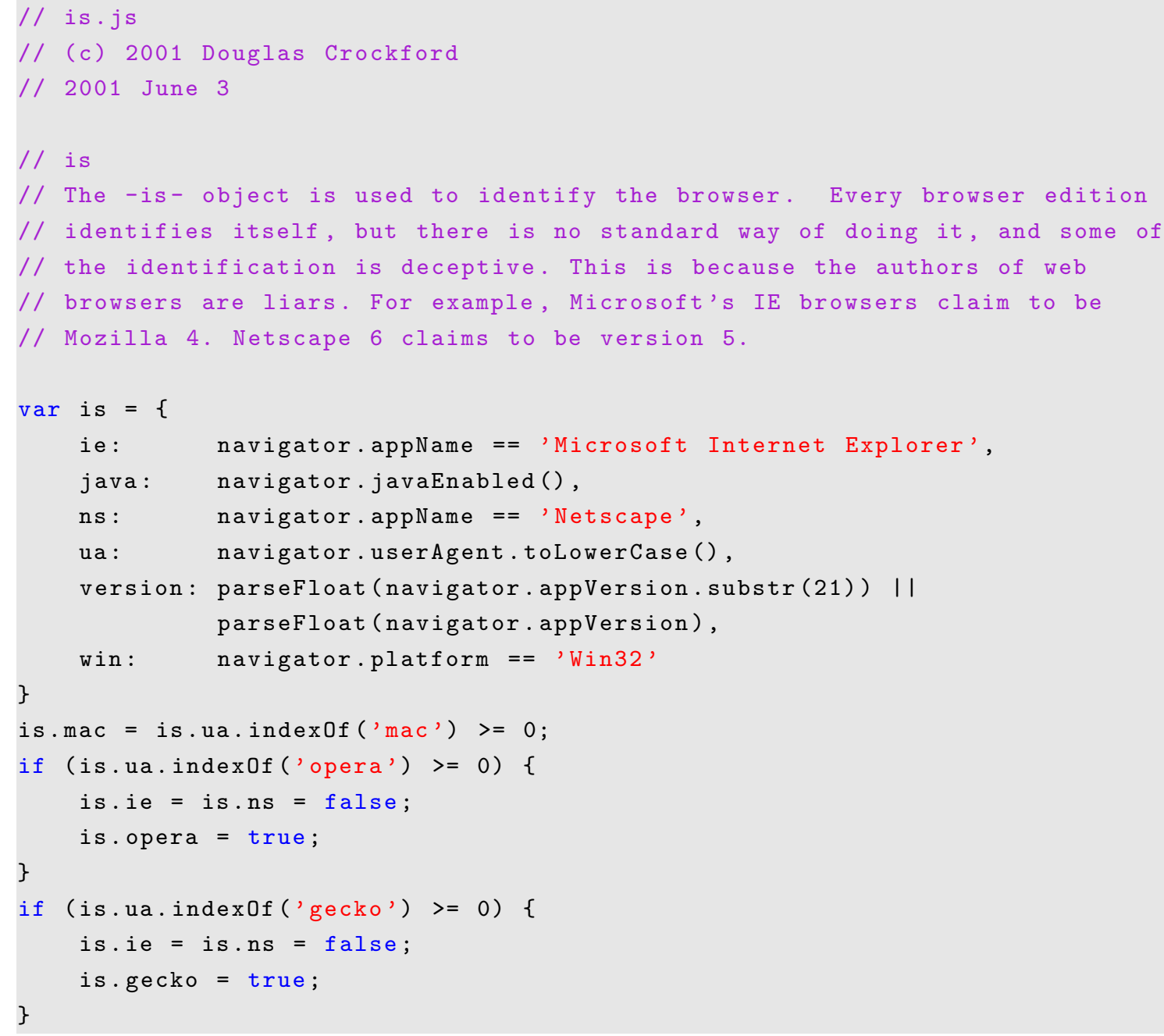

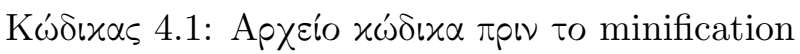

1 var is=\{ie:navigator. appName=='Microsoft Internet Explorer', java:navigator. javaEnabled (), ns: navigator. appName==' Netscape' , ua: navigator. userAgent. toLowerCase (), version: parseFloat (navigator.appVersion.substr(21))।I parsefloat (navigator. appVersion), win: navigator.platform=='Win32'\}

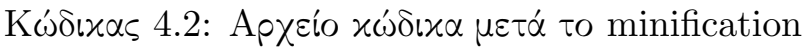

A

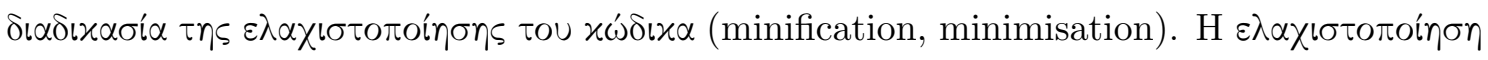

${ }^{2}$ https://github.com/github/linguist/ 


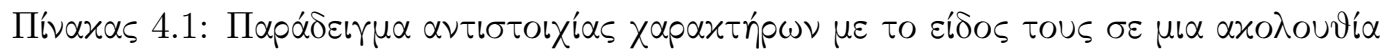

\begin{tabular}{|c|c|c|c|c|c|c|c|c|c|c|c|c|c|c|c|c|c|c|c|c|c|}
\hline String 1 & $\mathrm{v}$ & $\mathrm{a}$ & $\mathrm{r}$ & & $\mathrm{a}$ & $=$ & 1 & ; & $\mathrm{f}$ & $\mathrm{u}$ & $\mathrm{n}$ & $\mathrm{c}$ & $\mathrm{t}$ & $\mathrm{i}$ & 0 & $\mathrm{n}$ & & $\mathrm{f}$ & & A & ) \\
\hline Label 1 & $\mathrm{~K}$ & $\mathrm{~K}$ & K & $\mathrm{P}$ & I & $\mathrm{O}$ & $\mathrm{N}$ & $\mathrm{P}$ & $\mathrm{K}$ & $\mathrm{K}$ & $\mathrm{K}$ & $\mathrm{K}$ & K & K & $\mathrm{K}$ & $\mathrm{K}$ & $\mathrm{P}$ & I & & I & $\mathrm{P}$ \\
\hline String 2 & \{ & $\mathrm{r}$ & e & $\mathrm{t}$ & $\mathrm{u}$ & $\mathrm{r}$ & $\mathrm{n}$ & & c & o & $\mathrm{k}$ & , & , & \} & $c$ & $=$ & $\mathrm{f}$ & 1 & & 0 & ) \\
\hline Label 2 & $\mathrm{P}$ & $\mathrm{K}$ & $\mathrm{K}$ & $\mathrm{K}$ & $\mathrm{K}$ & $\mathrm{K}$ & $\mathrm{K}$ & $\mathrm{P}$ & $\mathrm{S}$ & $\mathrm{S}$ & $\mathrm{S}$ & $\mathrm{S}$ & $\mathrm{P}$ & $\mathrm{P}$ & $\mathrm{I}$ & $\mathrm{O}$ & I & $\mathrm{P}$ & N & $\mathrm{N}$ & $\mathrm{P}$ \\
\hline
\end{tabular}

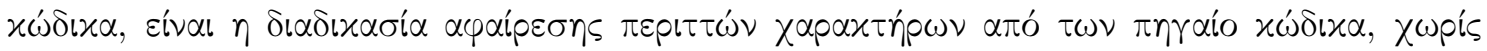

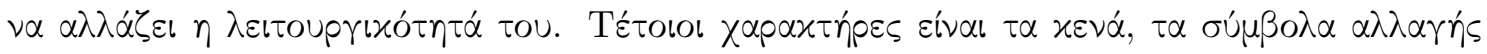

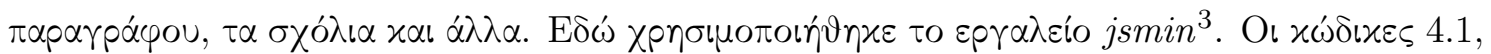

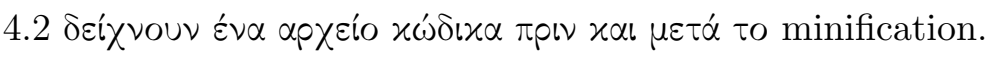

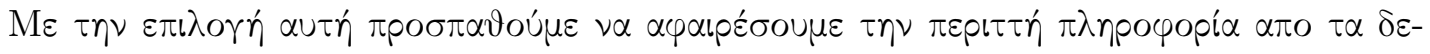

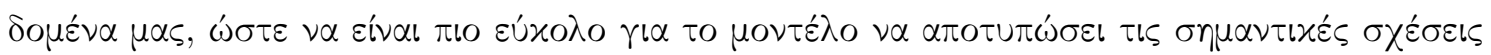

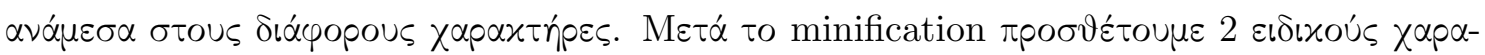

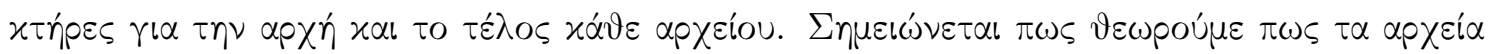

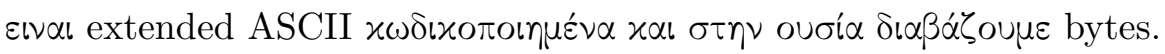

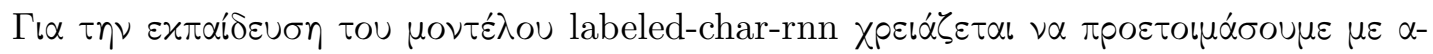

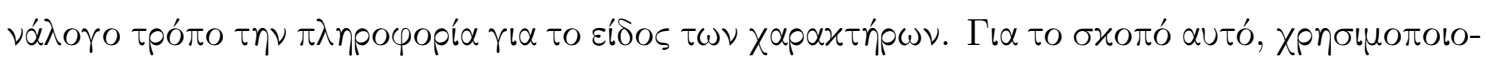

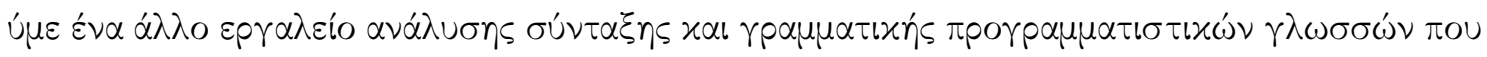

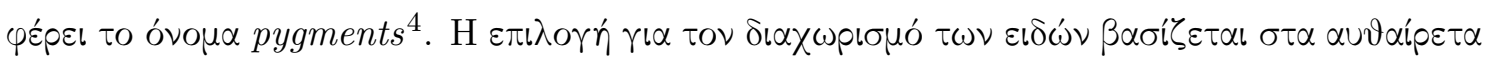

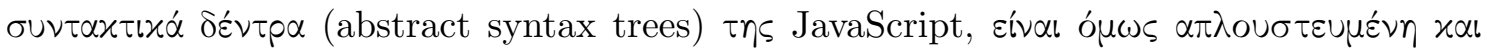

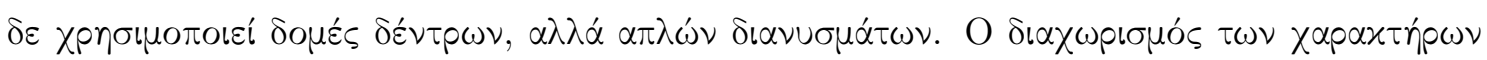

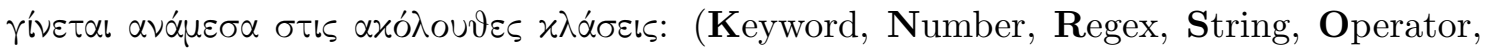
Punctuator, Identifier).

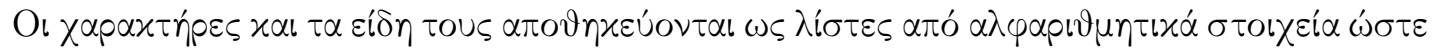

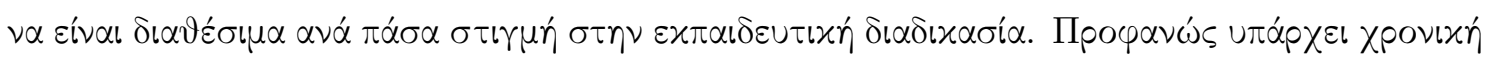

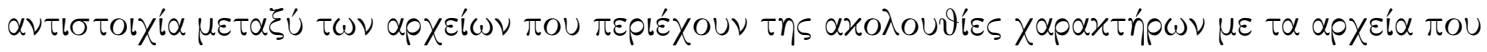

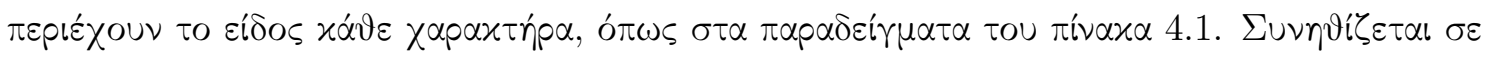

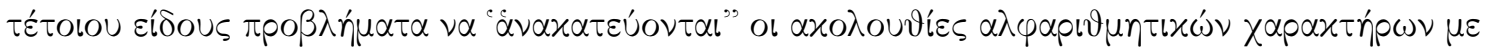

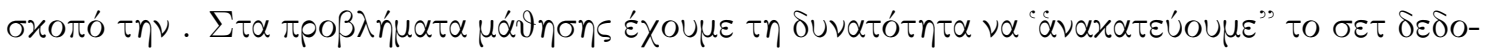

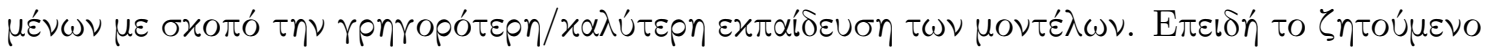

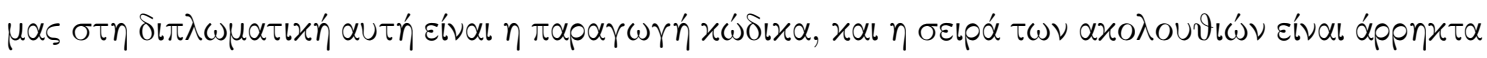

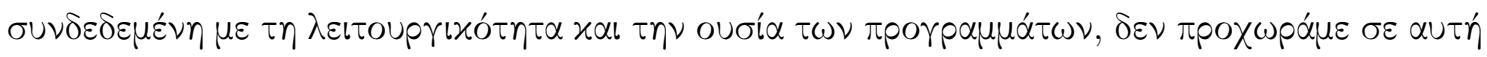

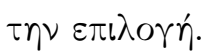

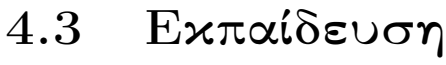

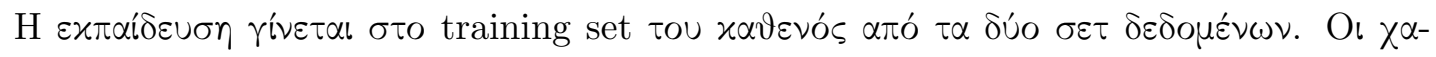

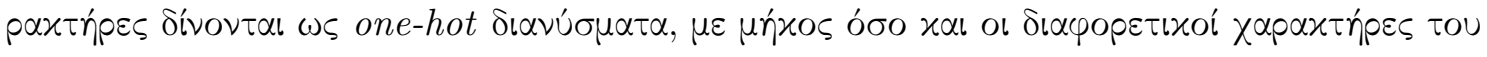

\footnotetext{
${ }^{3}$ http://www.crockford.com/javascript/jsmin.html

${ }^{4}$ http://pygments.org
} 


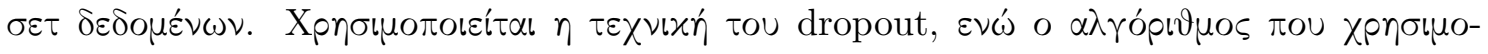

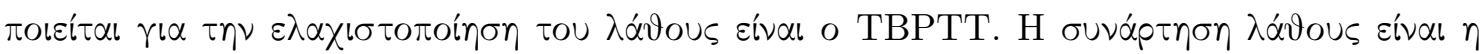

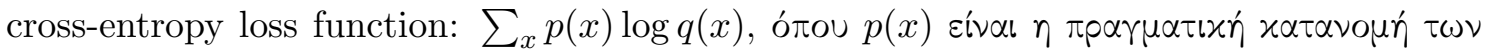

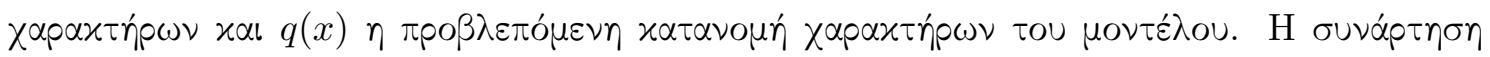

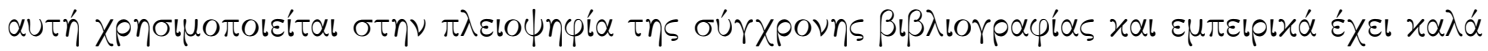

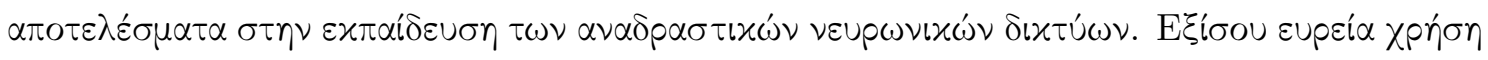

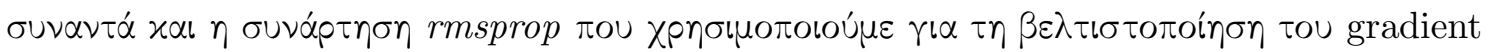
descent.

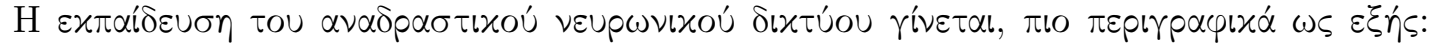

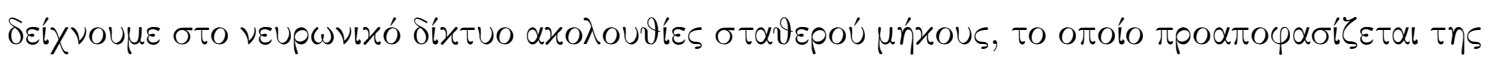

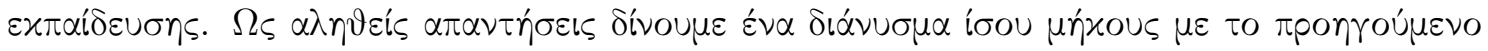

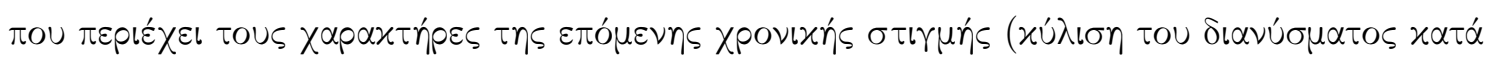

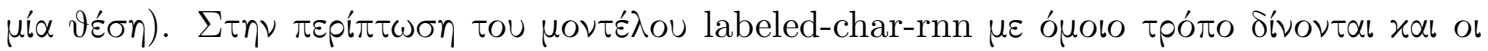

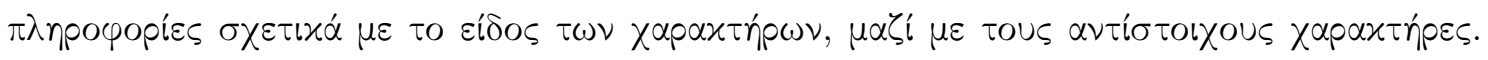

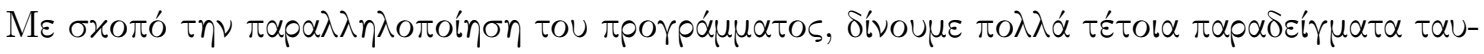
тóxpova.

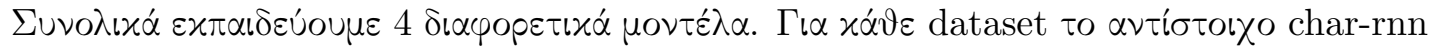

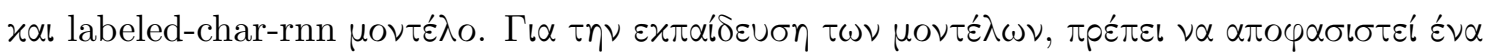

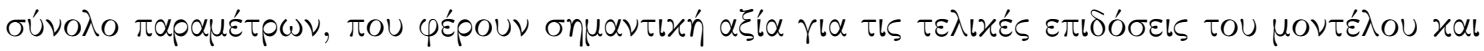

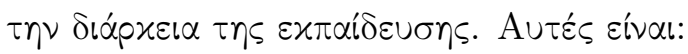

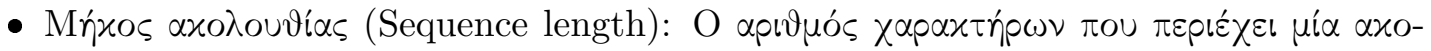
$\lambda$ ov७ía.

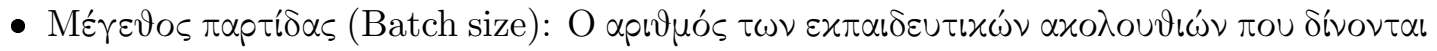

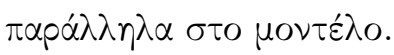

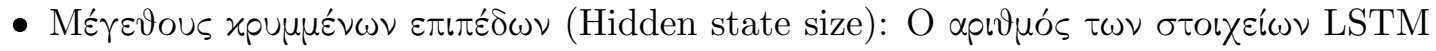

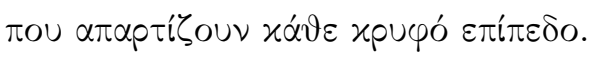

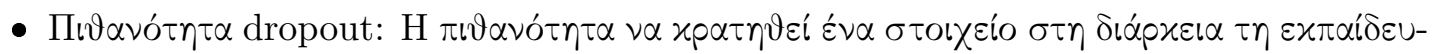
ons.

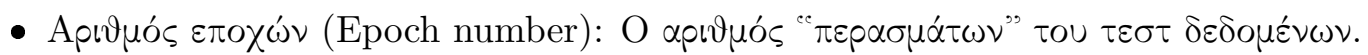

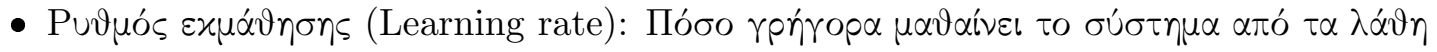
tou.

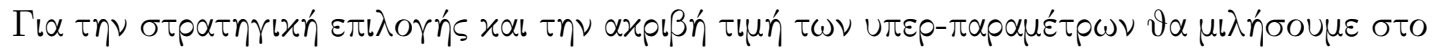

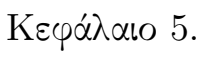

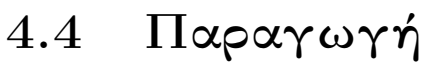

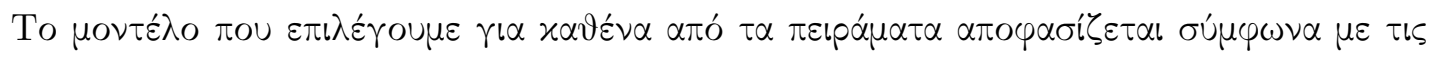

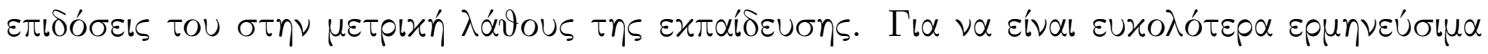




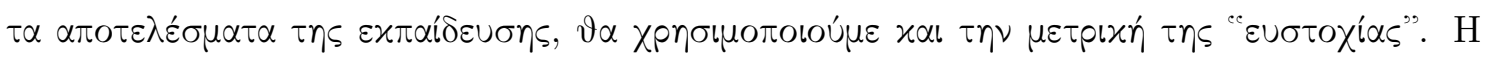

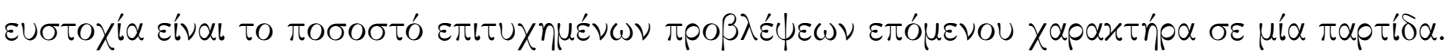

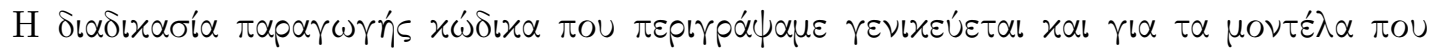

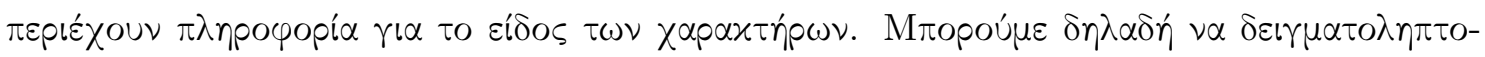

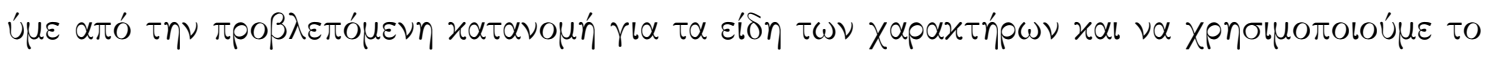

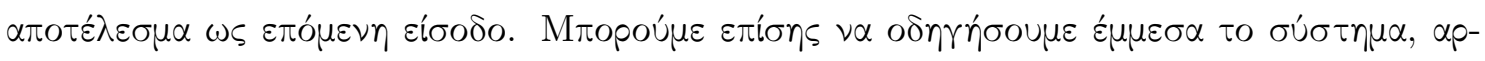

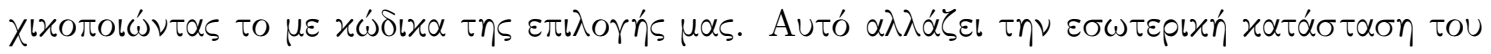

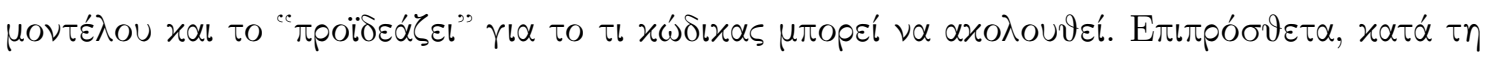

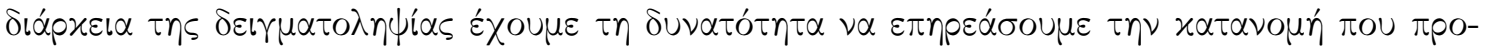

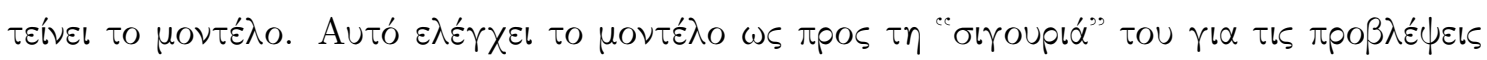

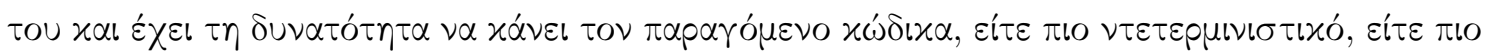

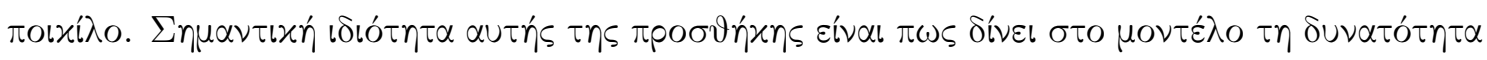

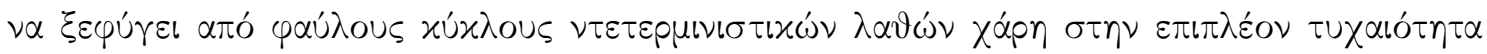

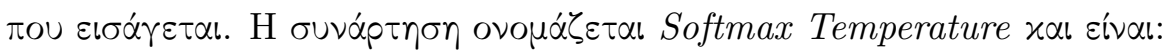

$$
P=\frac{e^{y / T}}{\sum_{k=1}^{n} e^{y_{k} / T}}
$$

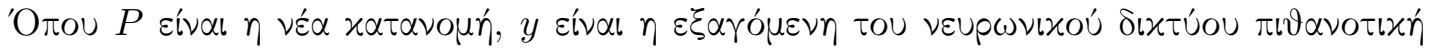

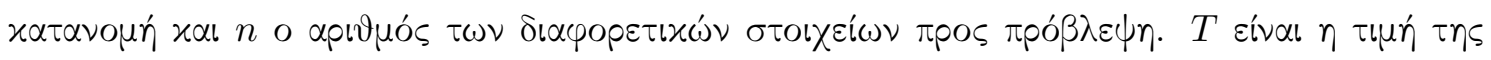

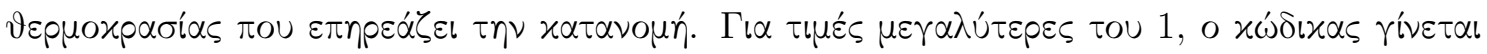

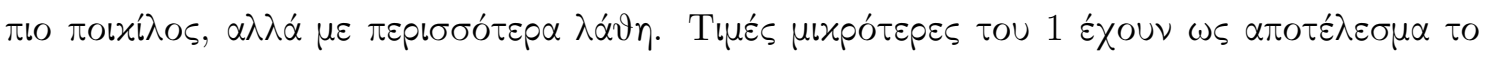

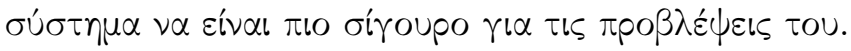





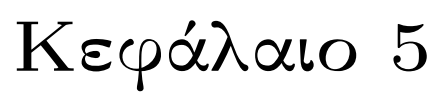

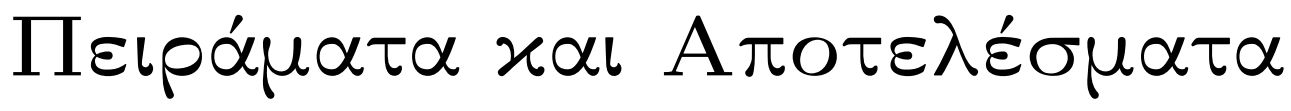

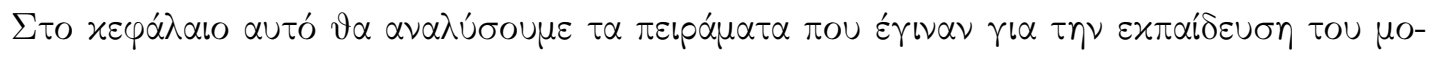

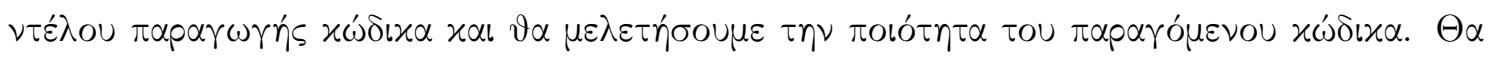

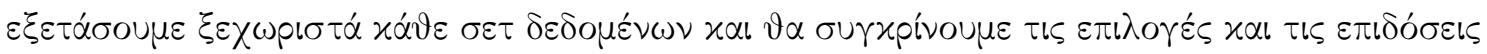

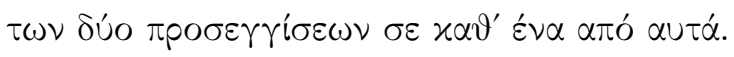

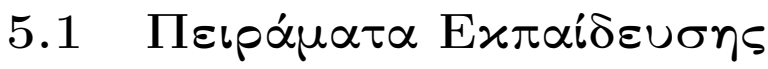

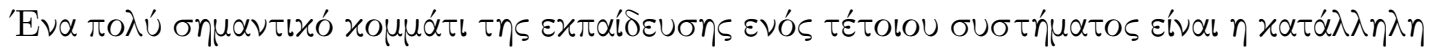

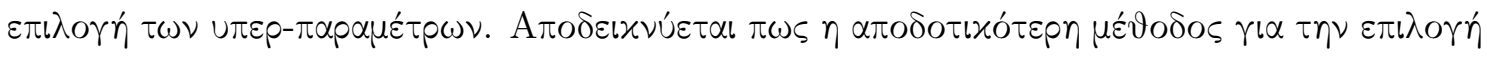

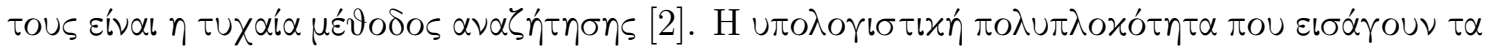

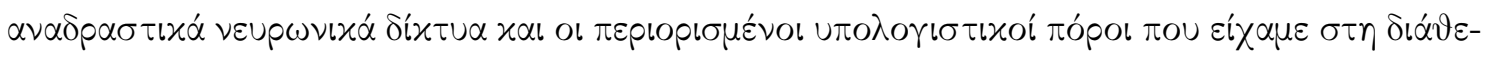

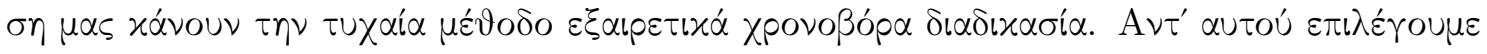

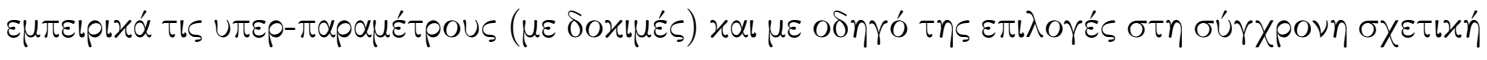

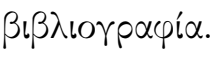

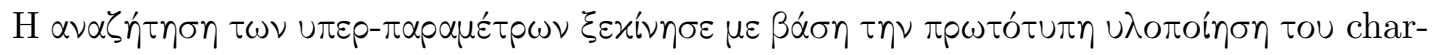

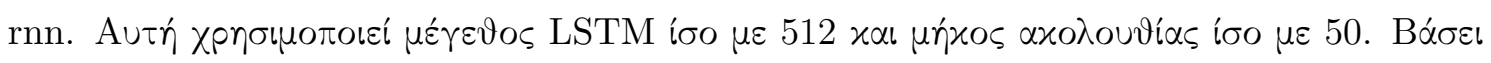

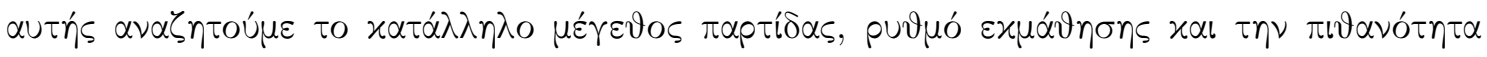

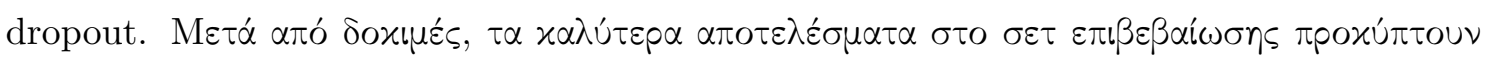

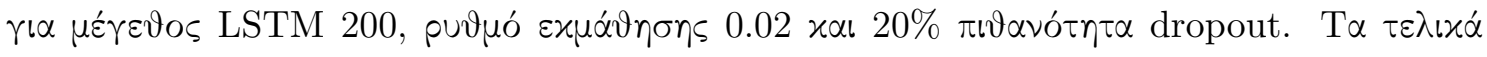

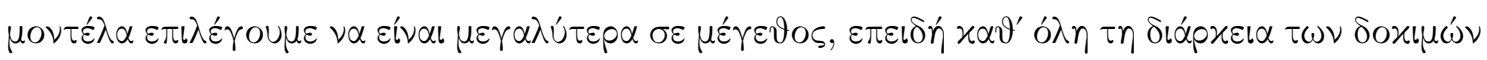

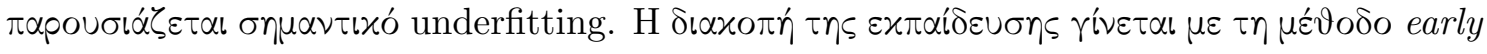

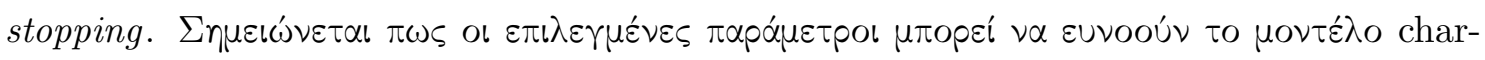

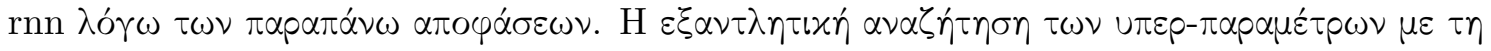

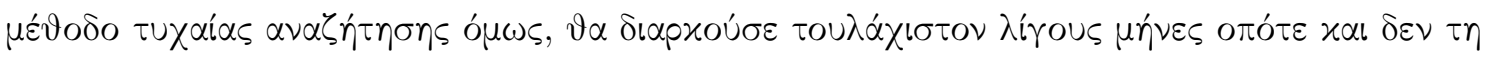

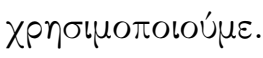




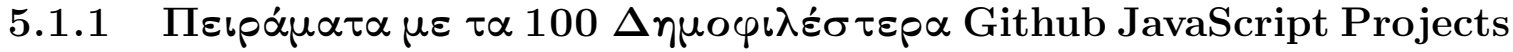

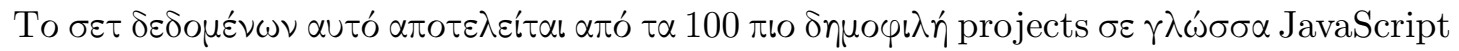

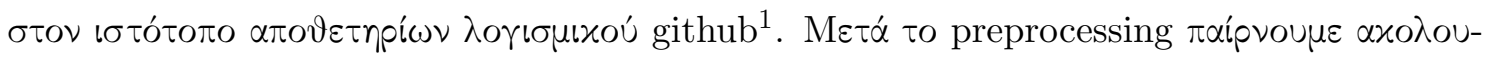

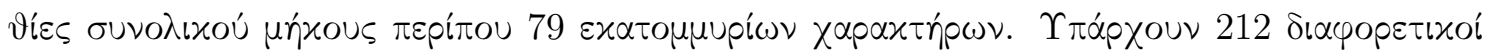

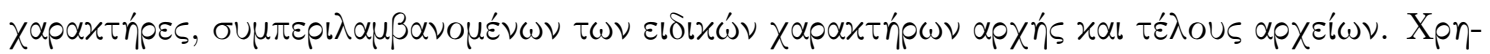

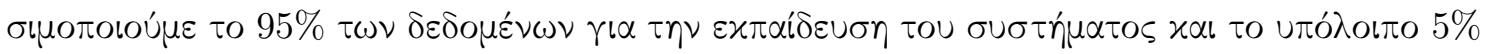

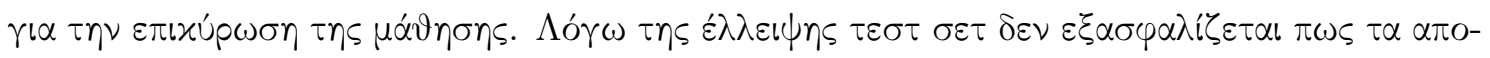

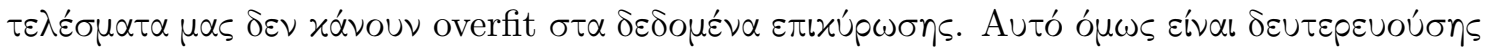

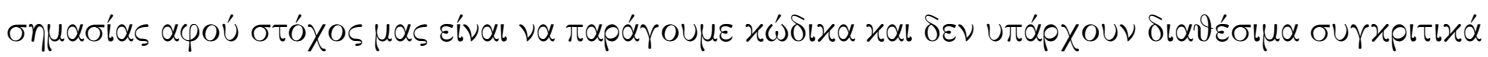

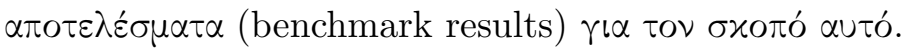

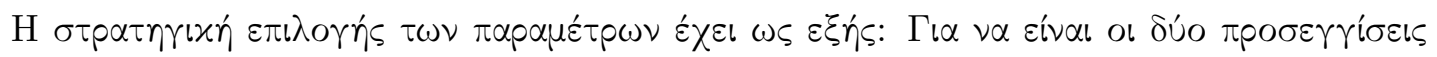

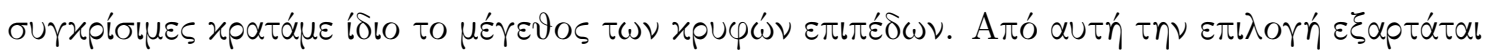

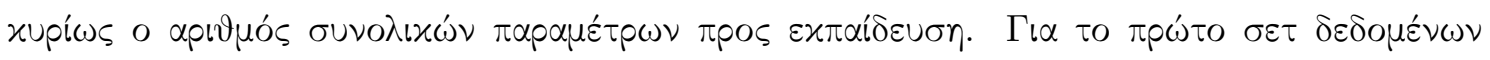

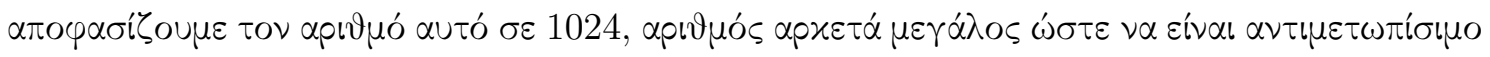

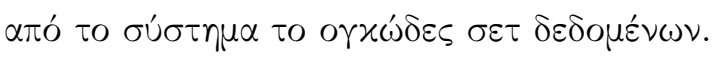

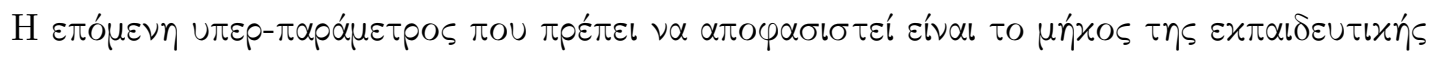

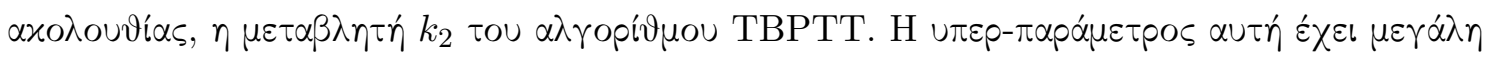

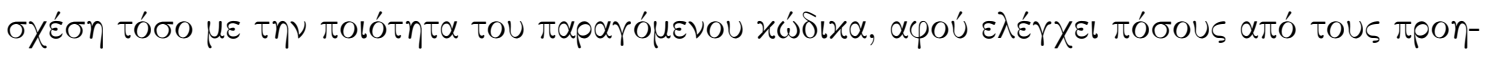

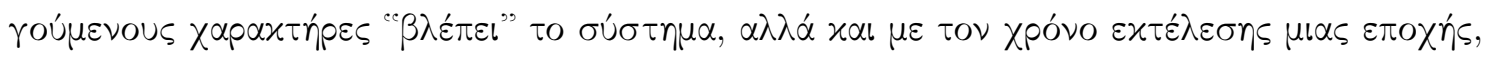

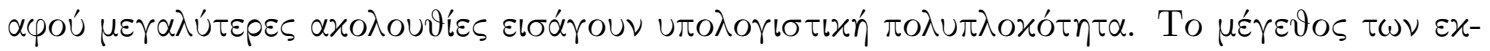

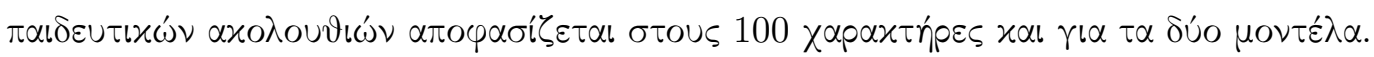

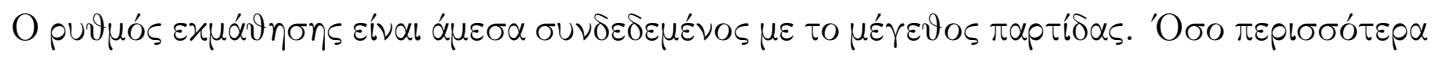

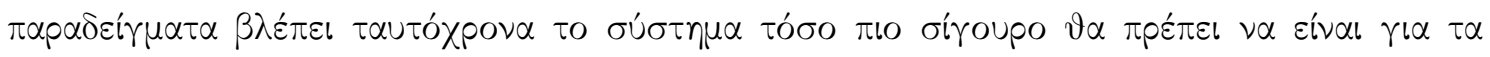

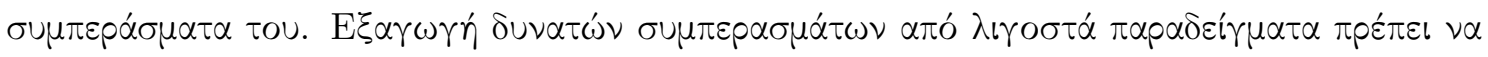

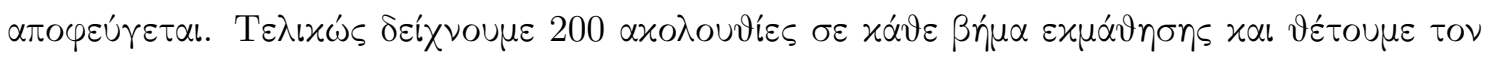

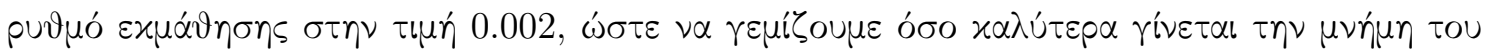

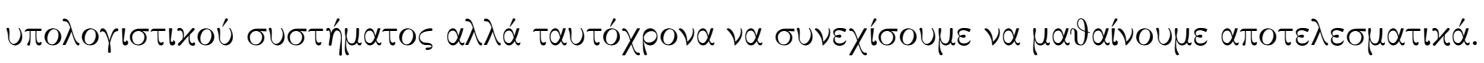

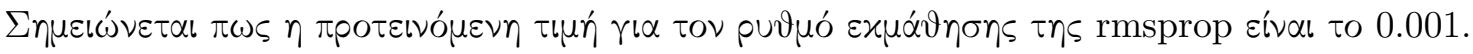

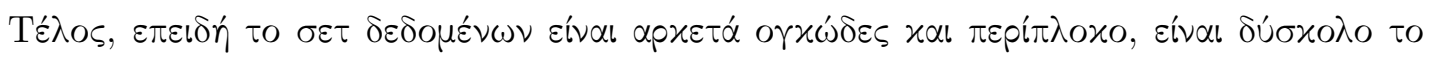

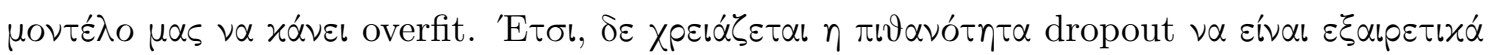

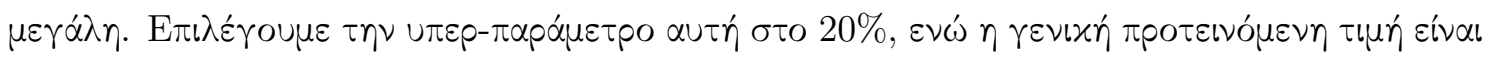

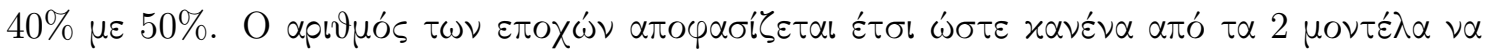

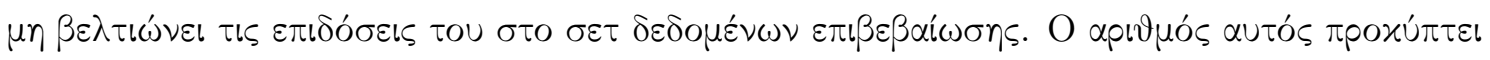

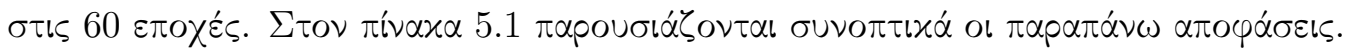

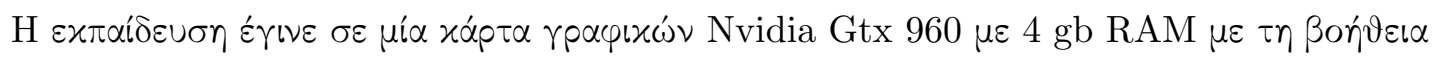

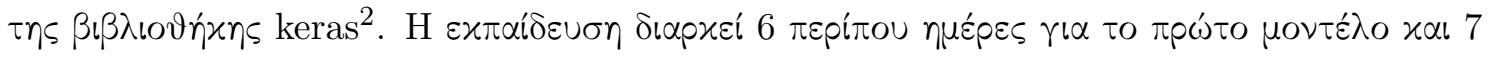

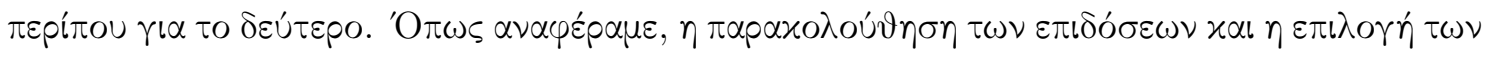

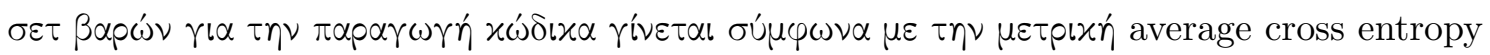

\footnotetext{
${ }^{1}$ ww. github.com

${ }^{2}$ https: //keras.io
} 


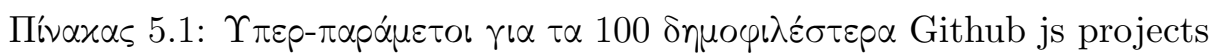

\begin{tabular}{|c|c|c|}
\hline & char-rnn & labeled-char-rnn \\
\hline 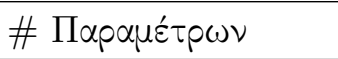 & $23 \mathrm{M}$ & $23 \mathrm{M}$ \\
\hline \# Xарахти́pav & 212 & 212,8 \\
\hline 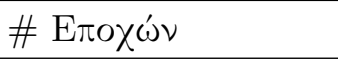 & 40 & 60 \\
\hline 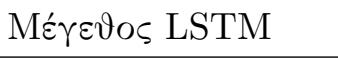 & 1024 & 1024 \\
\hline Mŕxos Axòov७ías & 100 & 100 \\
\hline 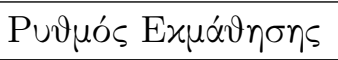 & 0.002 & 0.002 \\
\hline \% Dropout & 20 & 20 \\
\hline 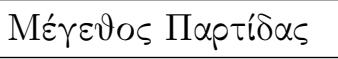 & 200 & 200 \\
\hline
\end{tabular}

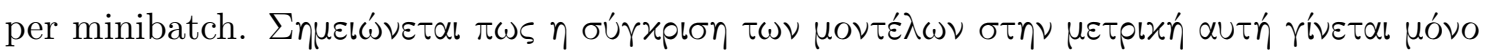

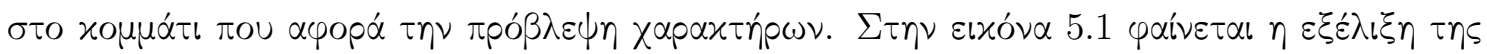

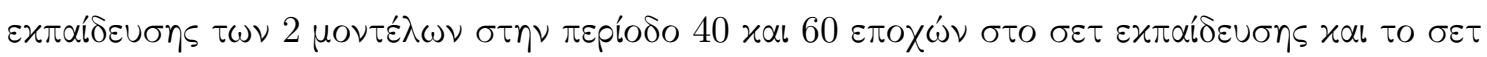

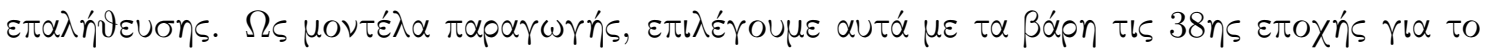

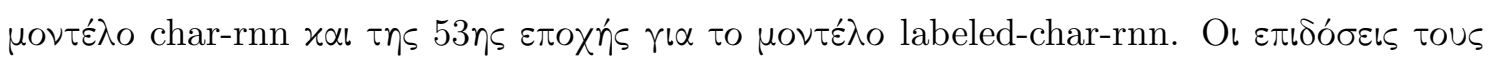

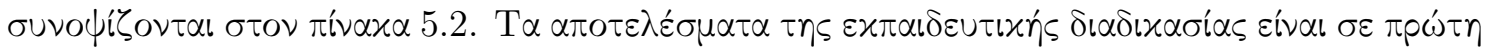

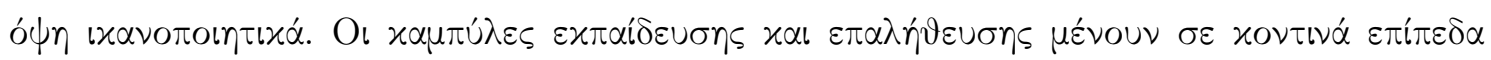

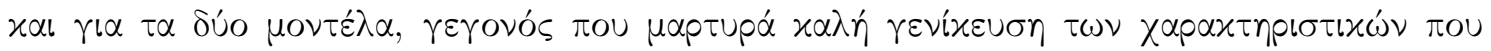

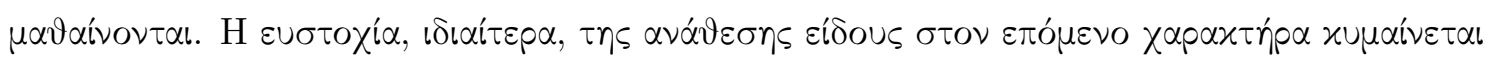
$\sigma \varepsilon \pi 0 \lambda \dot{~} \cup \psi \eta \lambda \dot{\alpha} \varepsilon \pi i \pi \varepsilon \delta \alpha$.

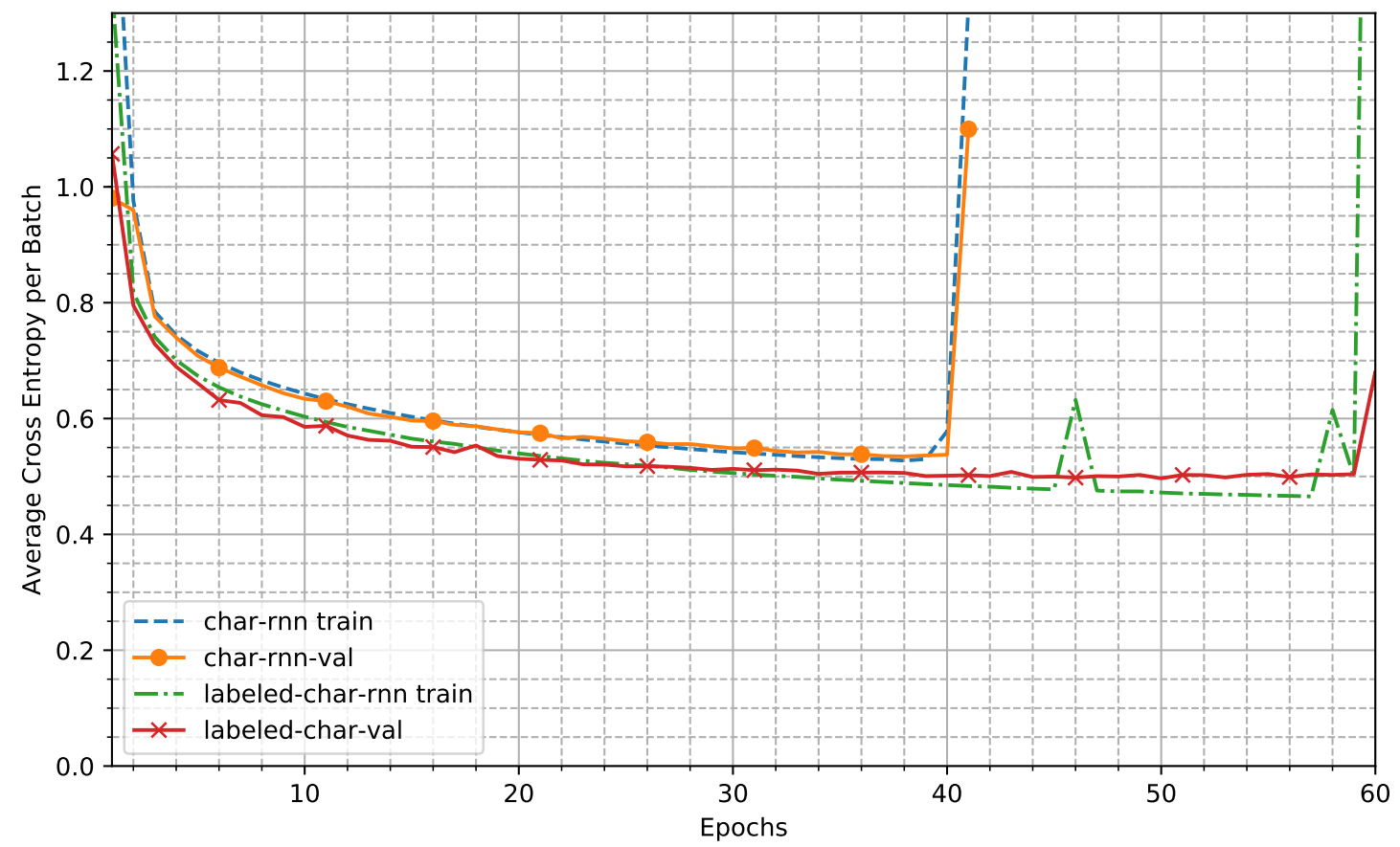

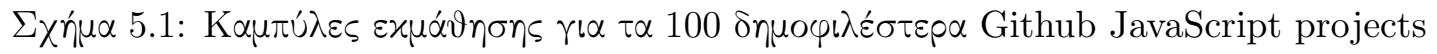




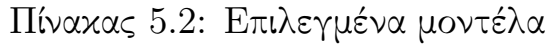

\begin{tabular}{|c|c|c|c|}
\hline & 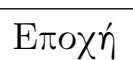 & 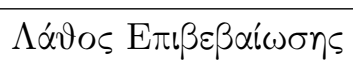 & 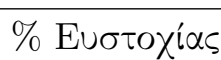 \\
\hline char-rnn & 38 & 0.534 & 85.6 \\
\hline labeled-char-rnn & 53 & $0.526,0.11$ & $87.2,97.1$ \\
\hline
\end{tabular}

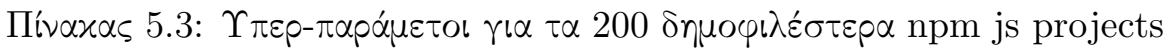

\begin{tabular}{|c|c|c|}
\hline & char-rnn & labeled-char-rnn \\
\hline 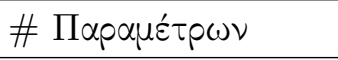 & $10 \mathrm{M}$ & $10 \mathrm{M}$ \\
\hline \# Xараxти́ран & 210 & 210,8 \\
\hline 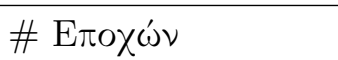 & 80 & 80 \\
\hline 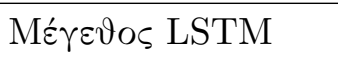 & 512 & 512 \\
\hline Mŕxos Axoגovyias & 100 & 100 \\
\hline 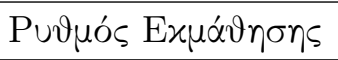 & 0.002 & 0.002 \\
\hline \% Dropout & 30 & 40 \\
\hline 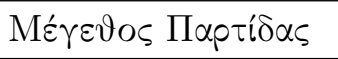 & 200 & 200 \\
\hline
\end{tabular}

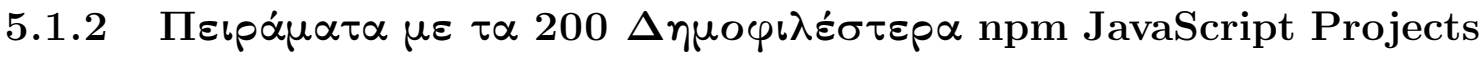

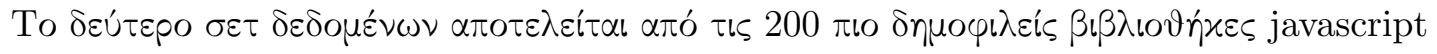

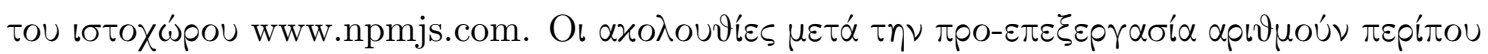

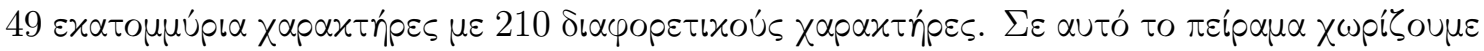

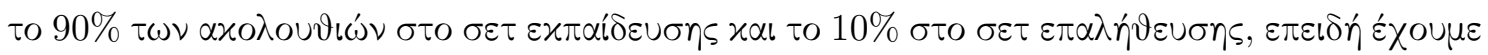

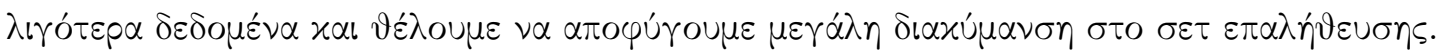

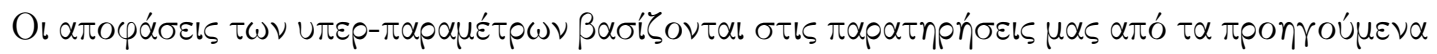

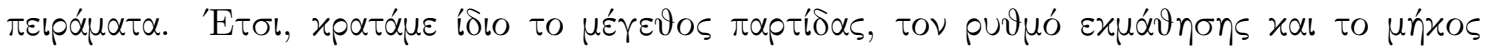

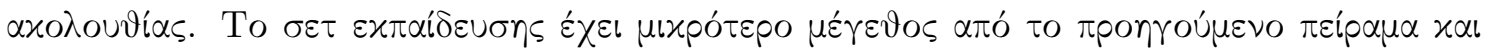

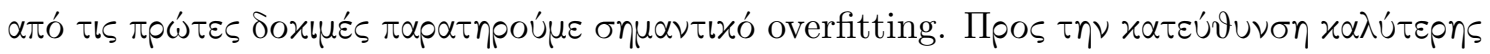

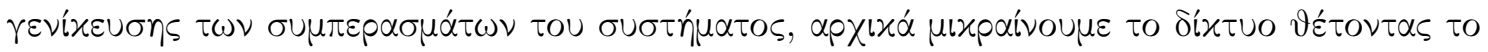

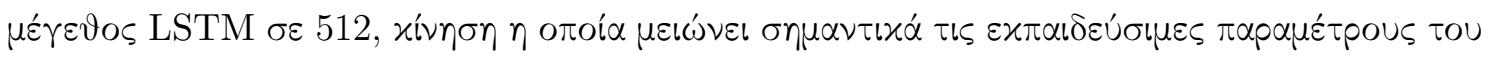

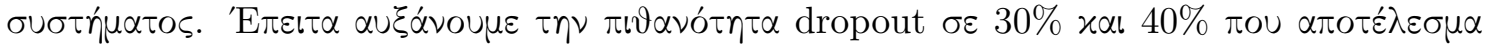

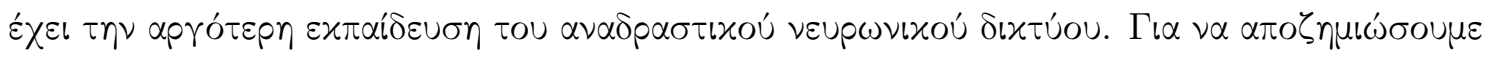

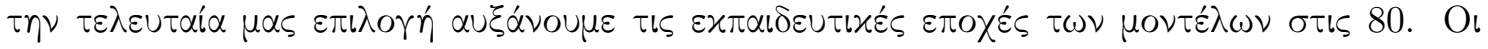

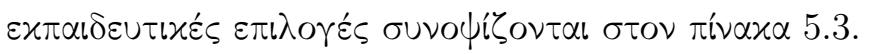

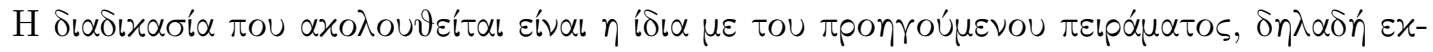

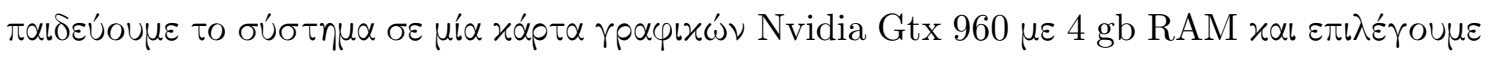

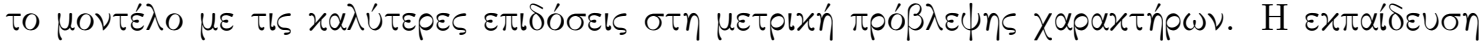

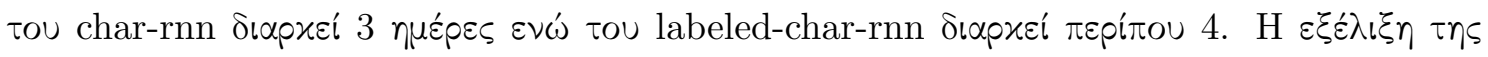

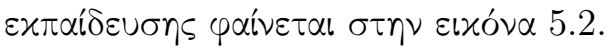

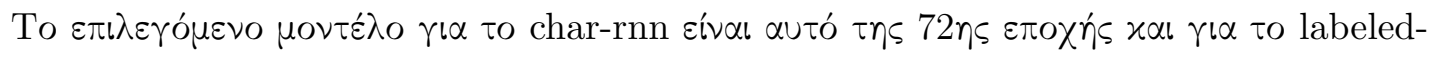

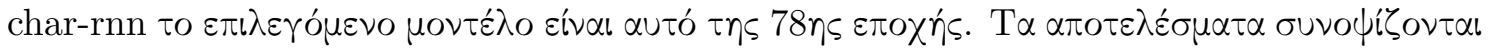




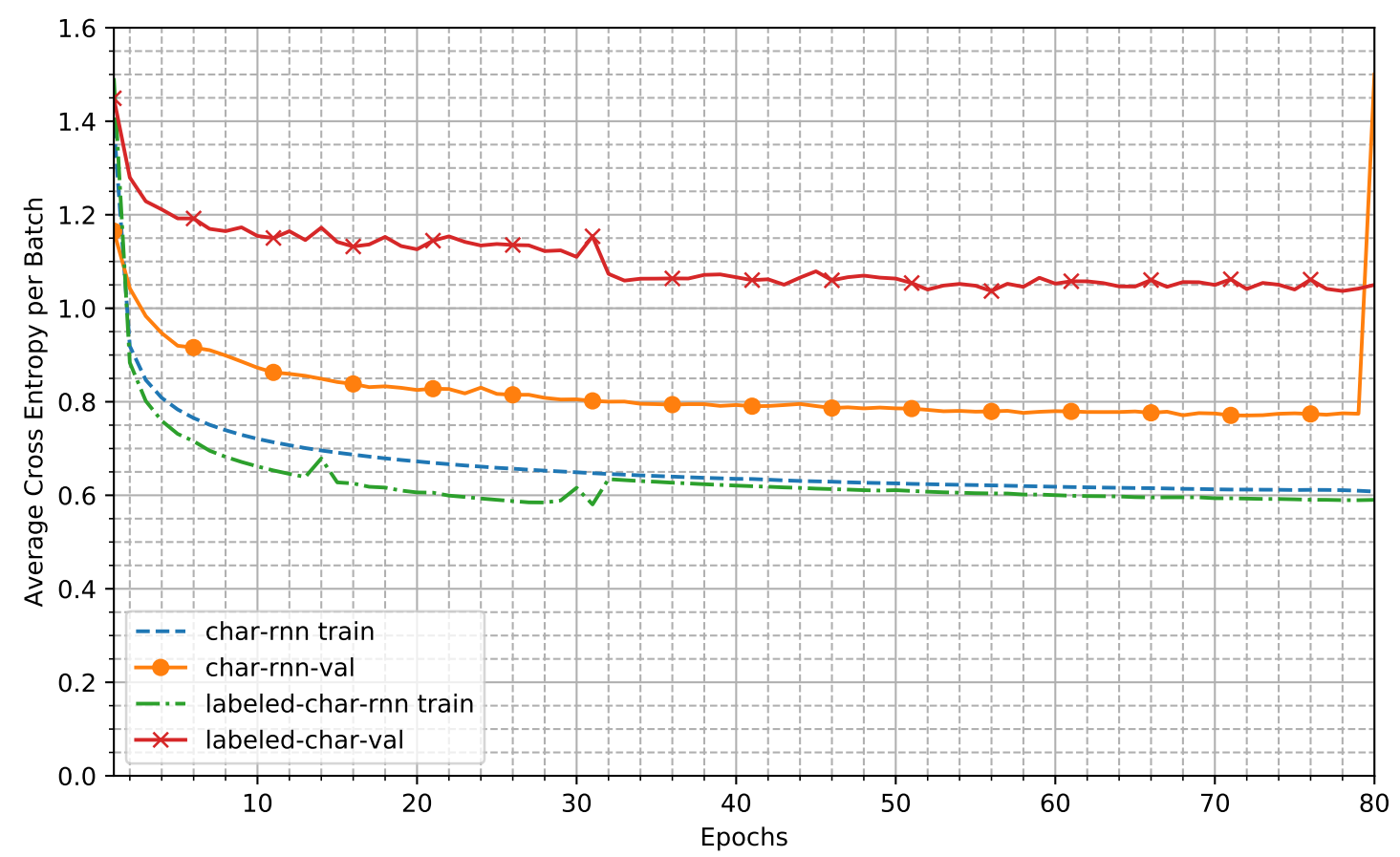

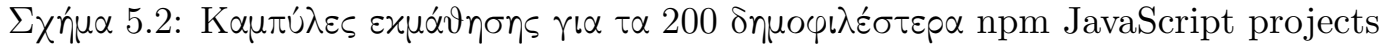

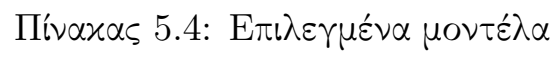

\begin{tabular}{|c|c|c|c|}
\hline & Eлoхń & 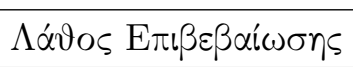 & $\%$ Evotoxias \\
\hline char-rnn & 72 & 0.771 & 78.9 \\
\hline labeled-char-rnn & 78 & $1.038,0.161$ & $72.3,94.7$ \\
\hline
\end{tabular}

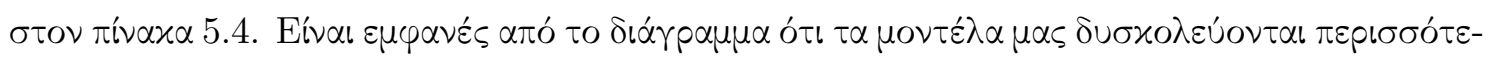

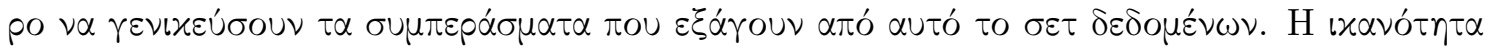

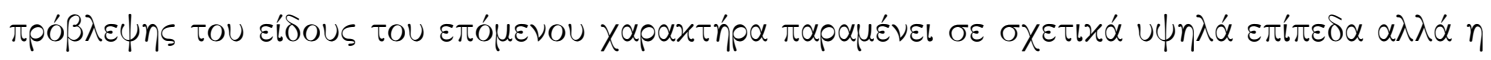

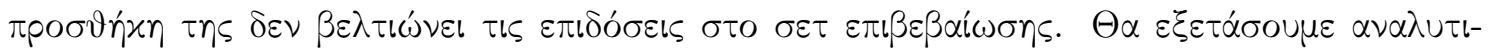

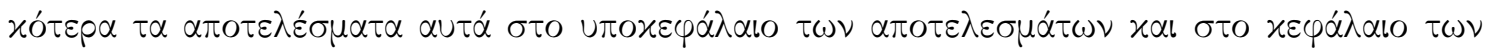
$\sigma u \mu \pi \varepsilon \rho \alpha \sigma \mu \alpha \dot{\tau} \omega \nu$.

\subsection{A $\pi 0 \tau \varepsilon \lambda \varepsilon \dot{\varepsilon} \sigma \mu \alpha \tau \alpha$}

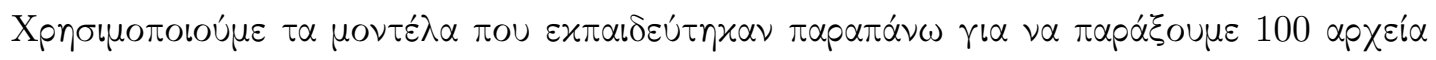

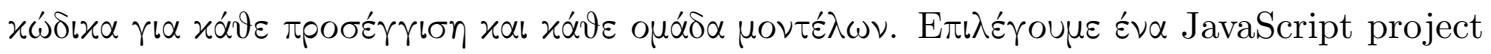

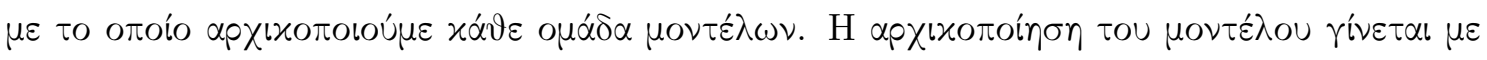

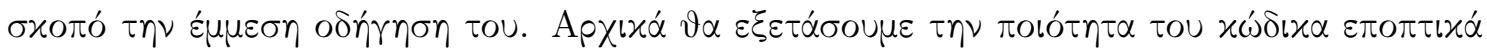

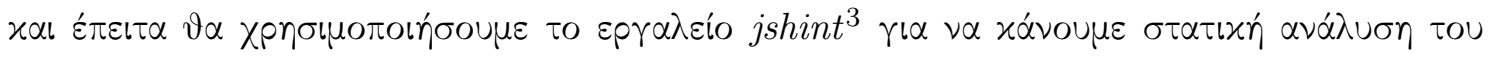
$x \omega \dot{\delta} \delta \varkappa \alpha$.

\footnotetext{
${ }^{3}$ http://jshint.com
} 


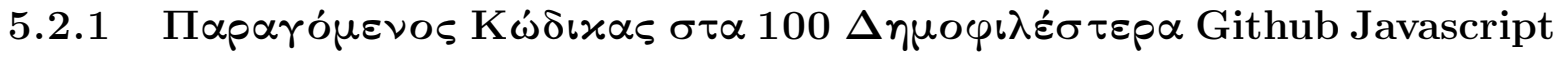 Projects}

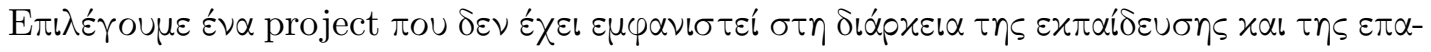

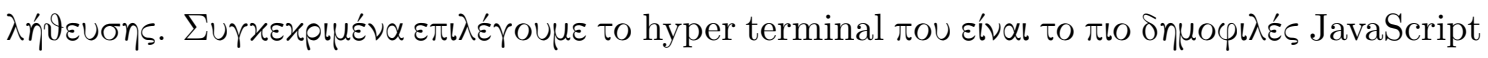

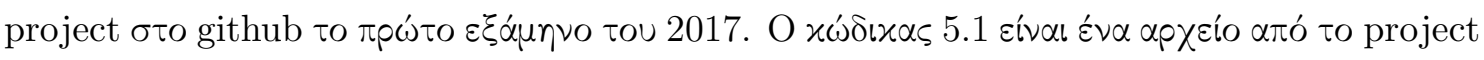
autó.

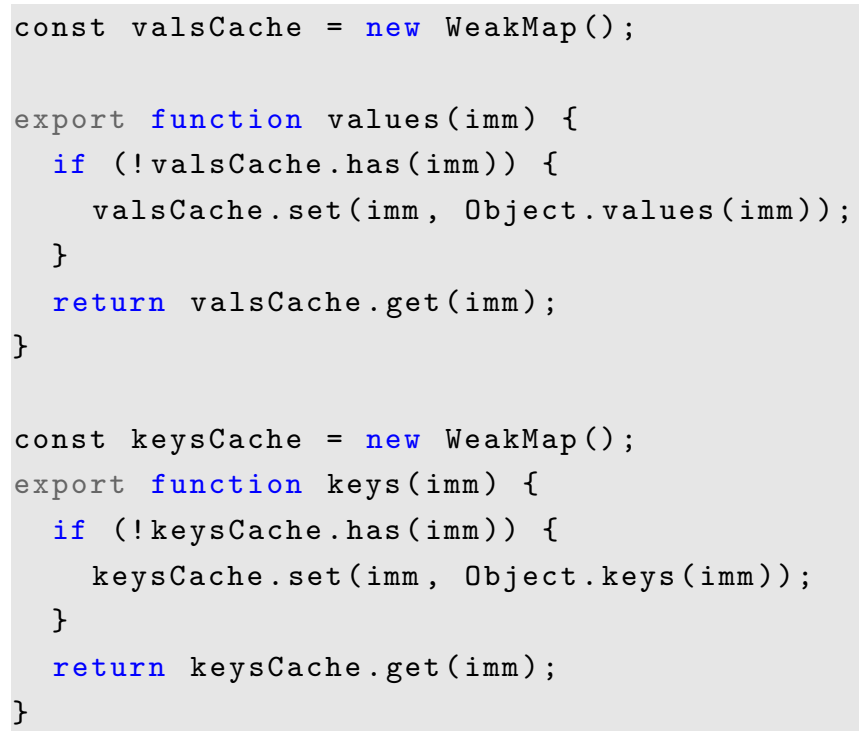

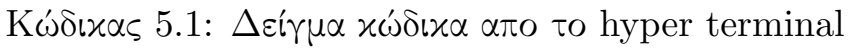

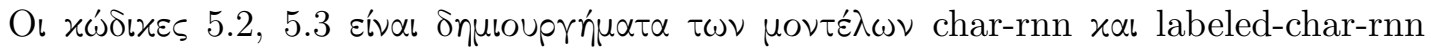

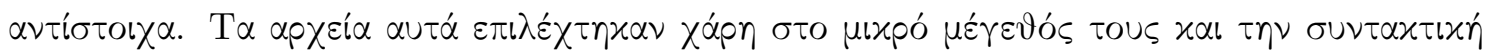

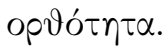

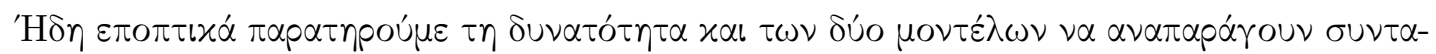

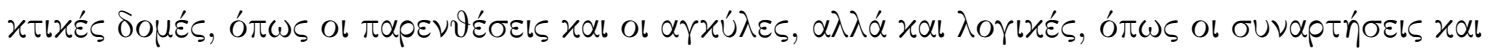

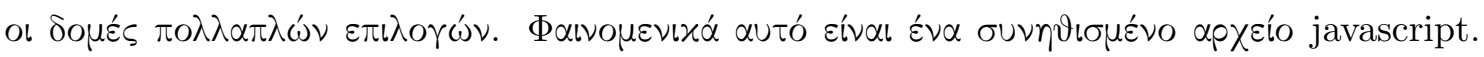

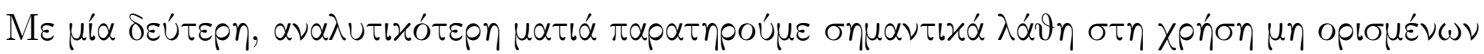

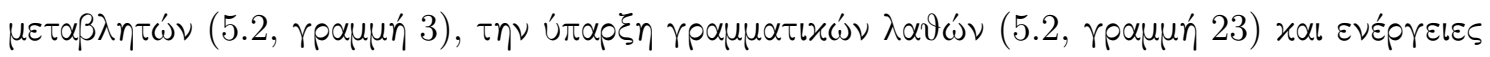

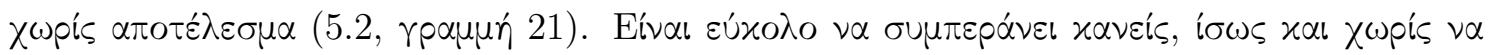

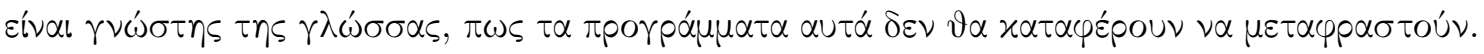




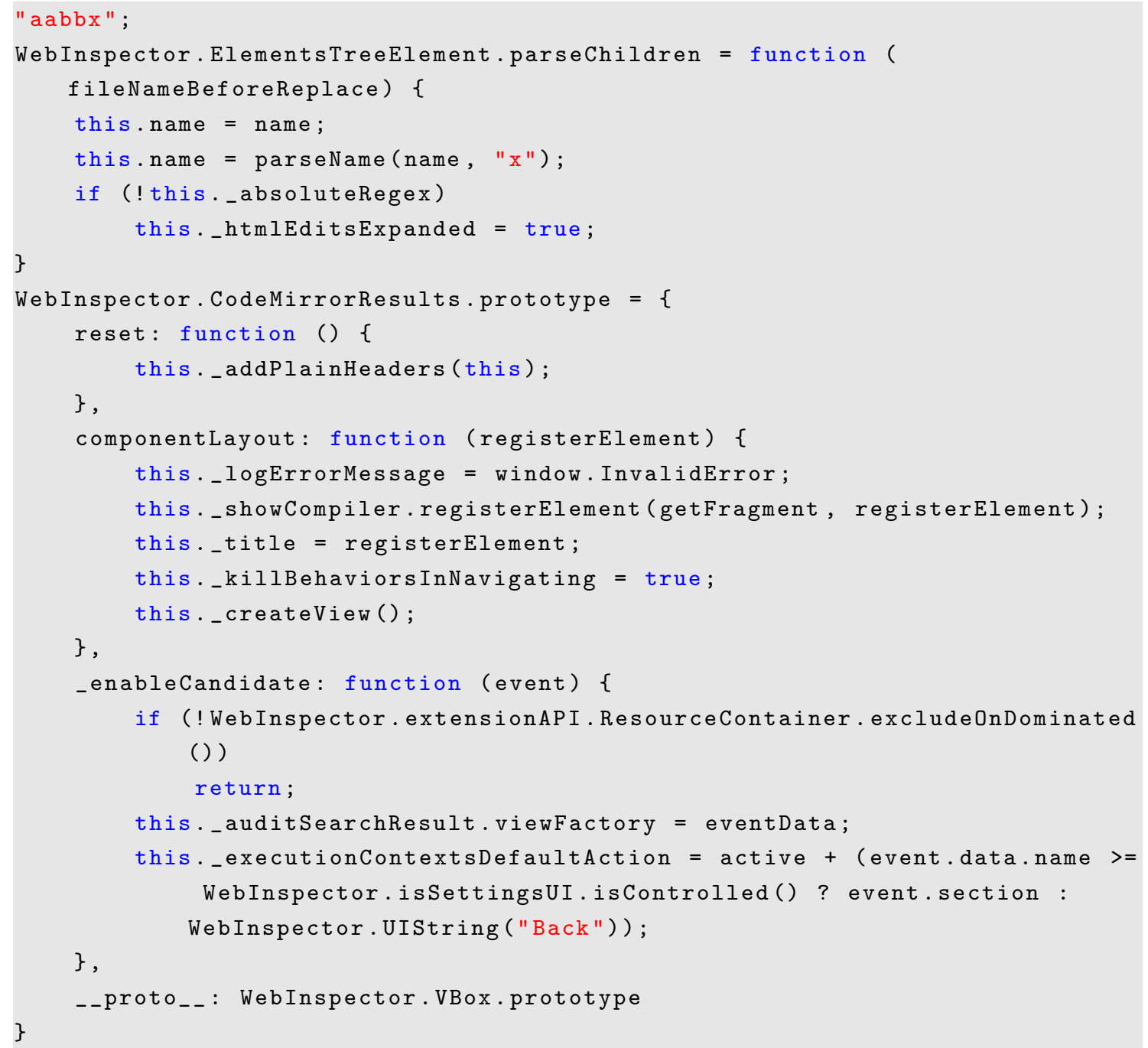

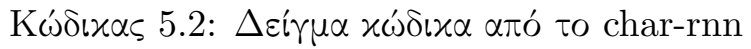

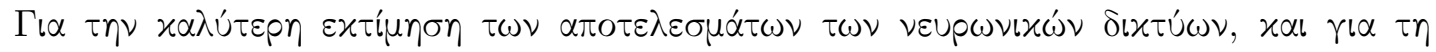

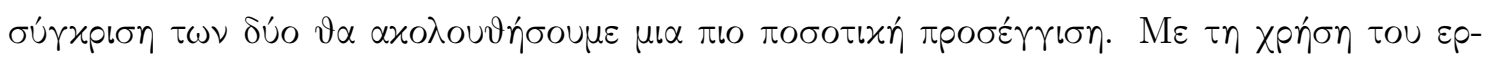

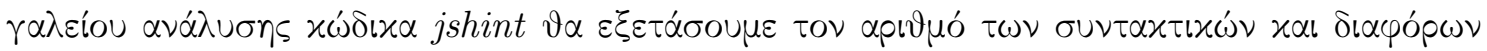

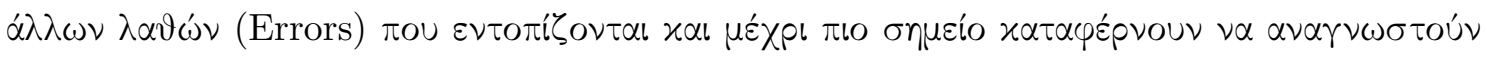

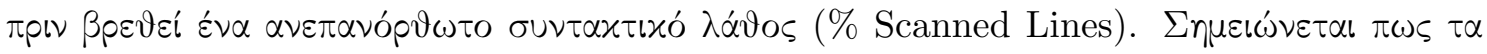

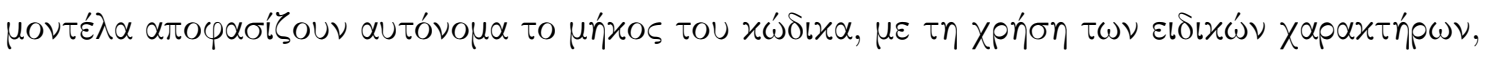

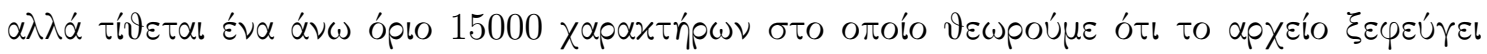

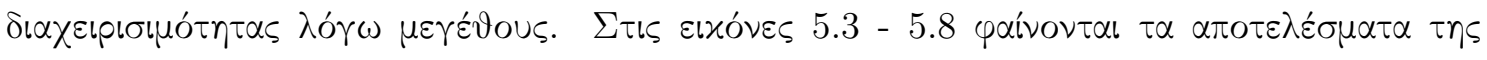

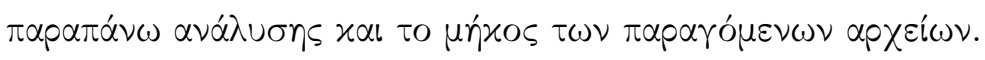




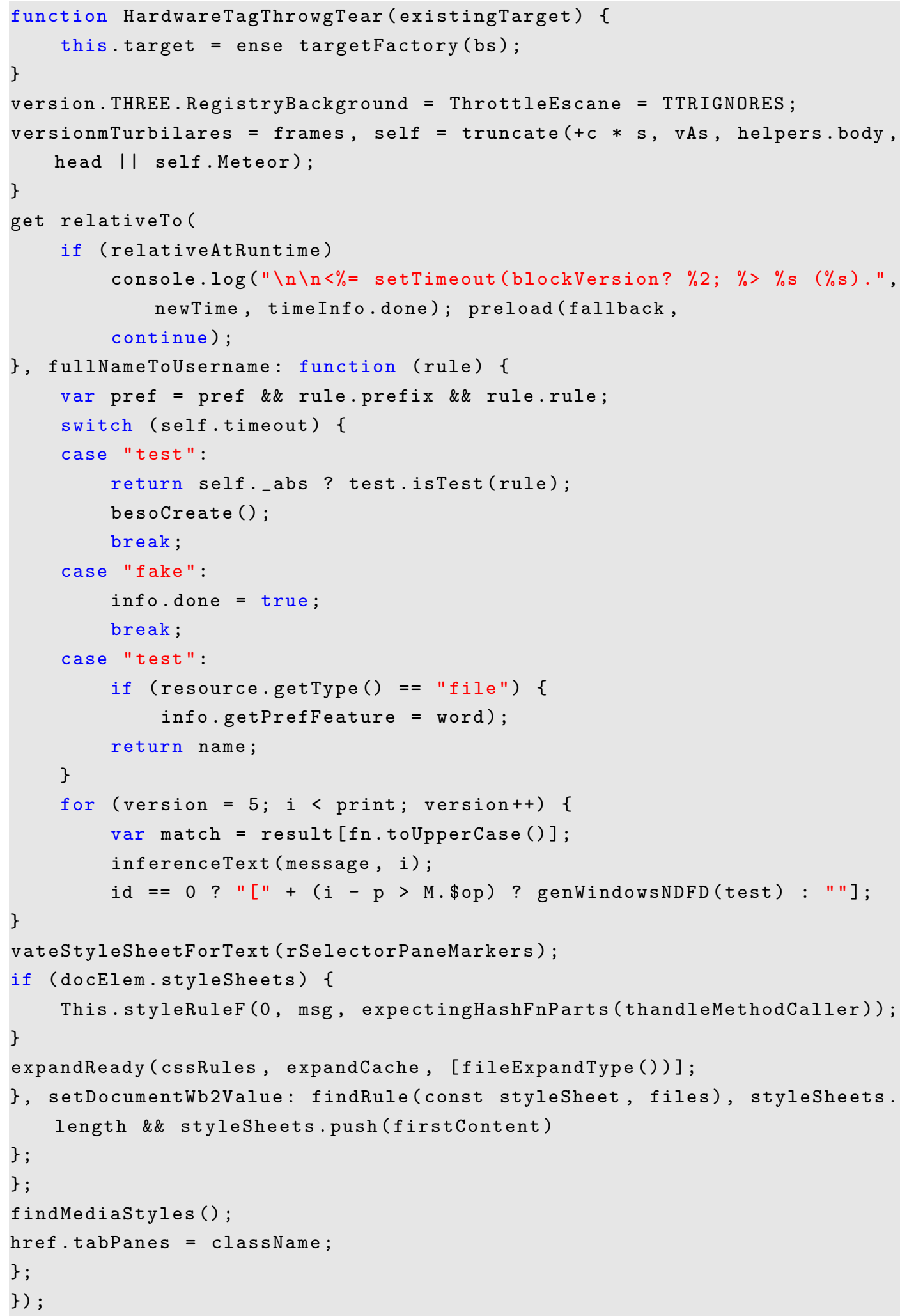

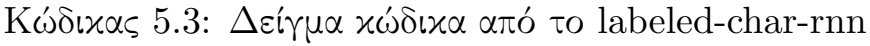

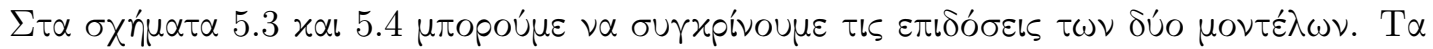

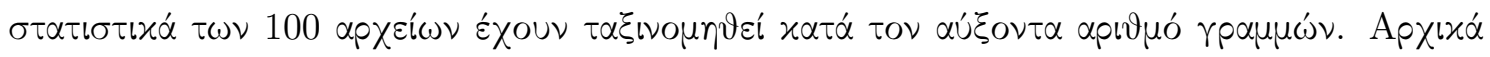

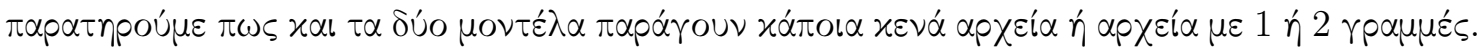




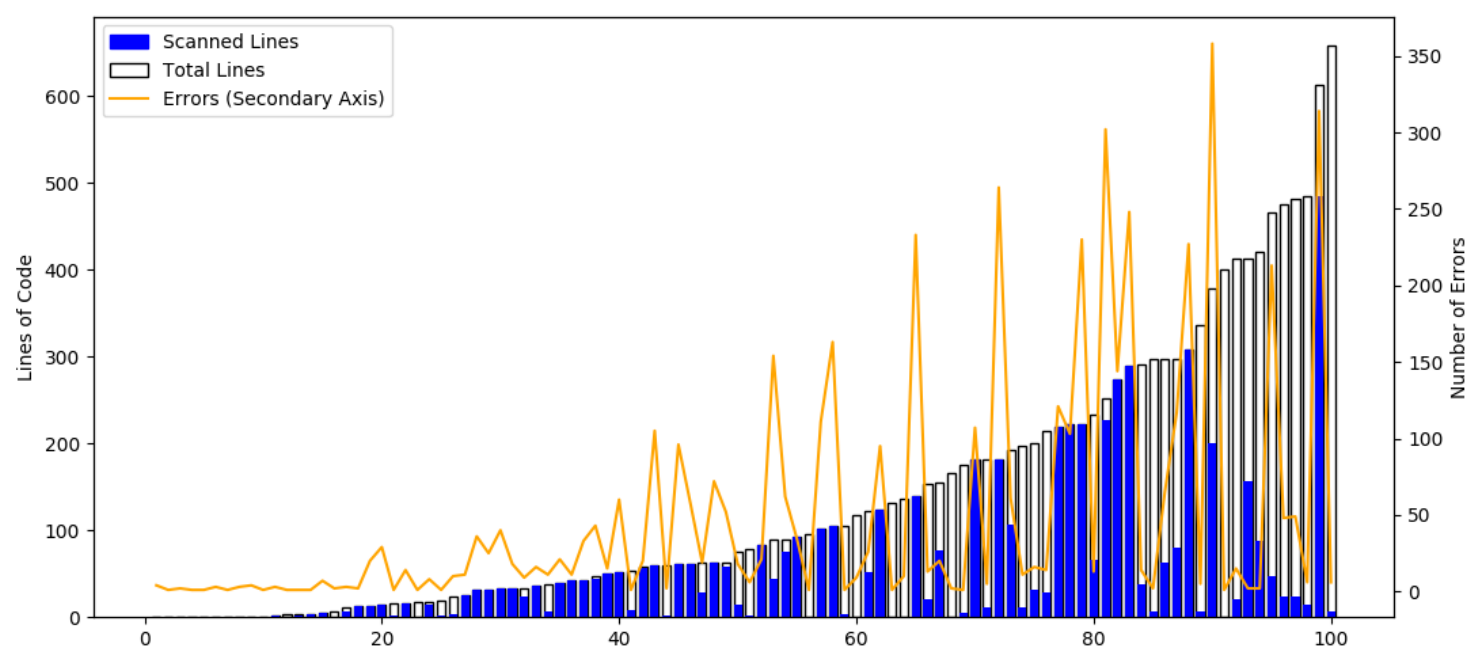

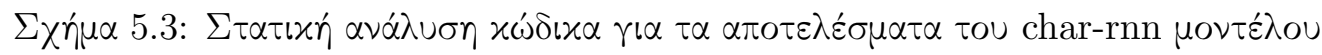

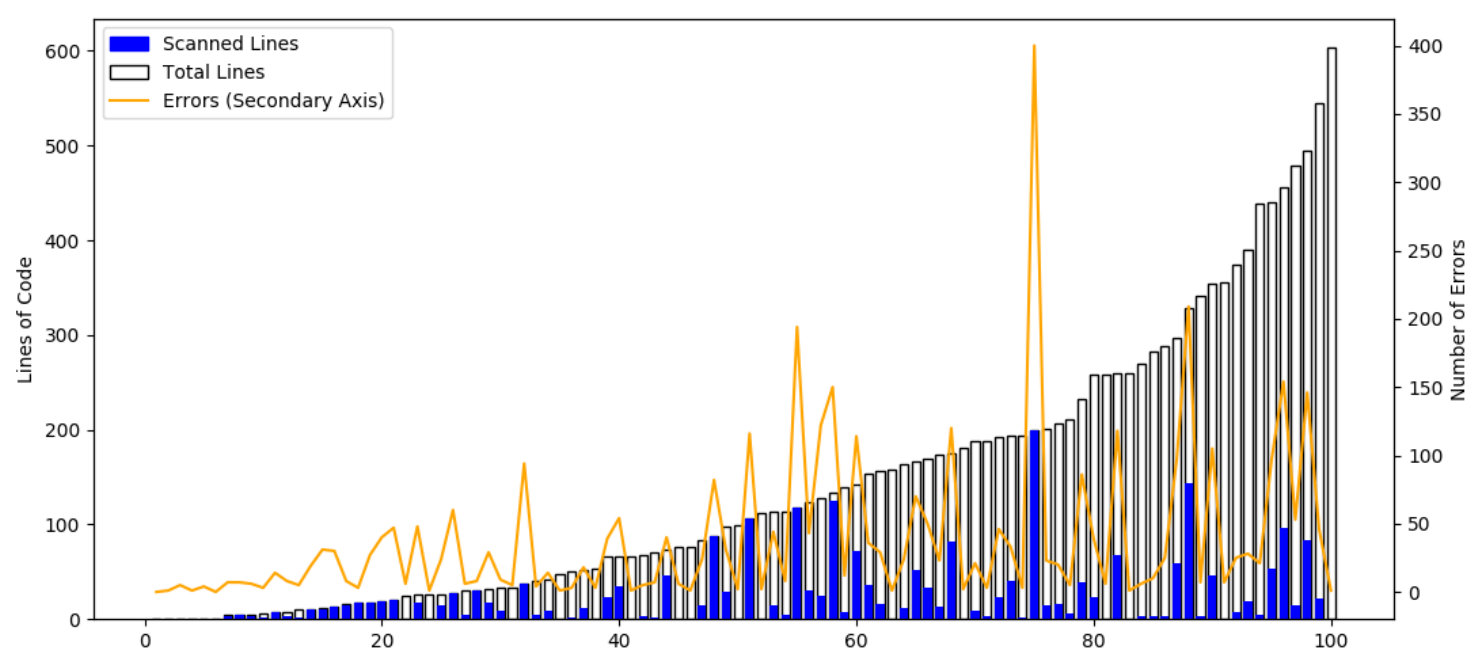

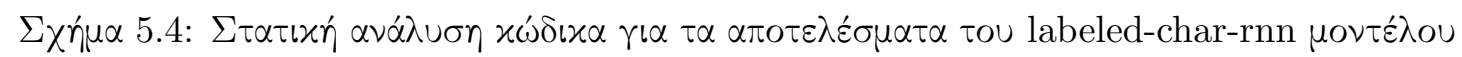

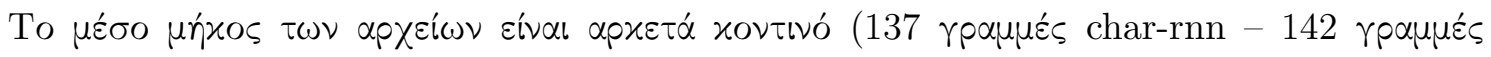

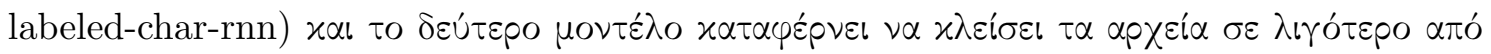

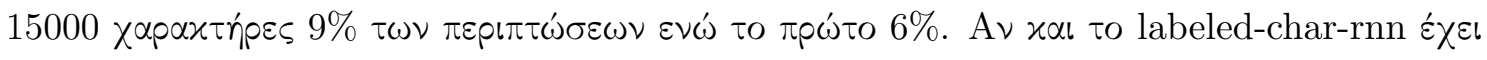

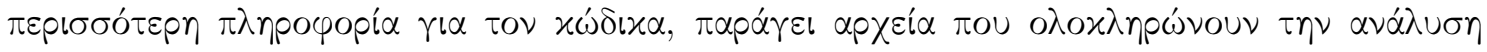

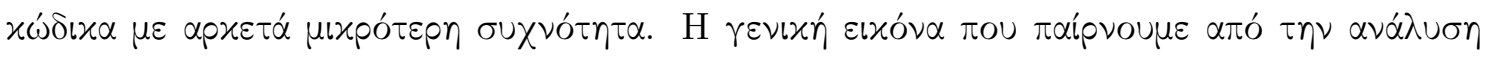

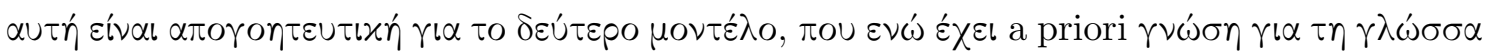

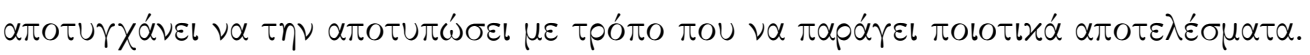

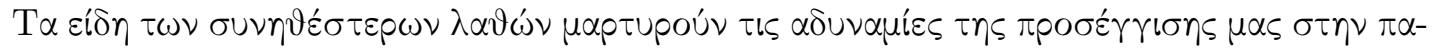

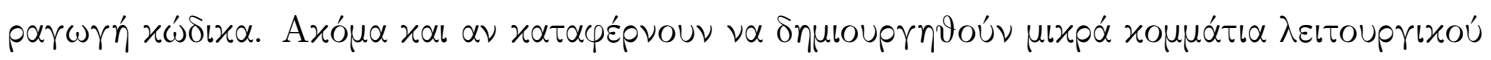

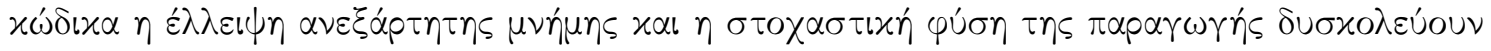

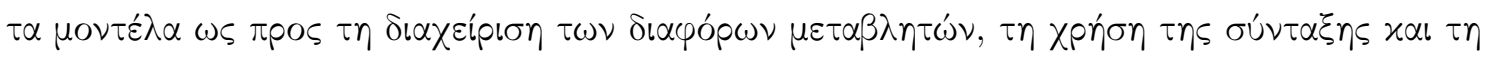

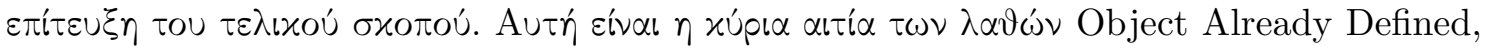




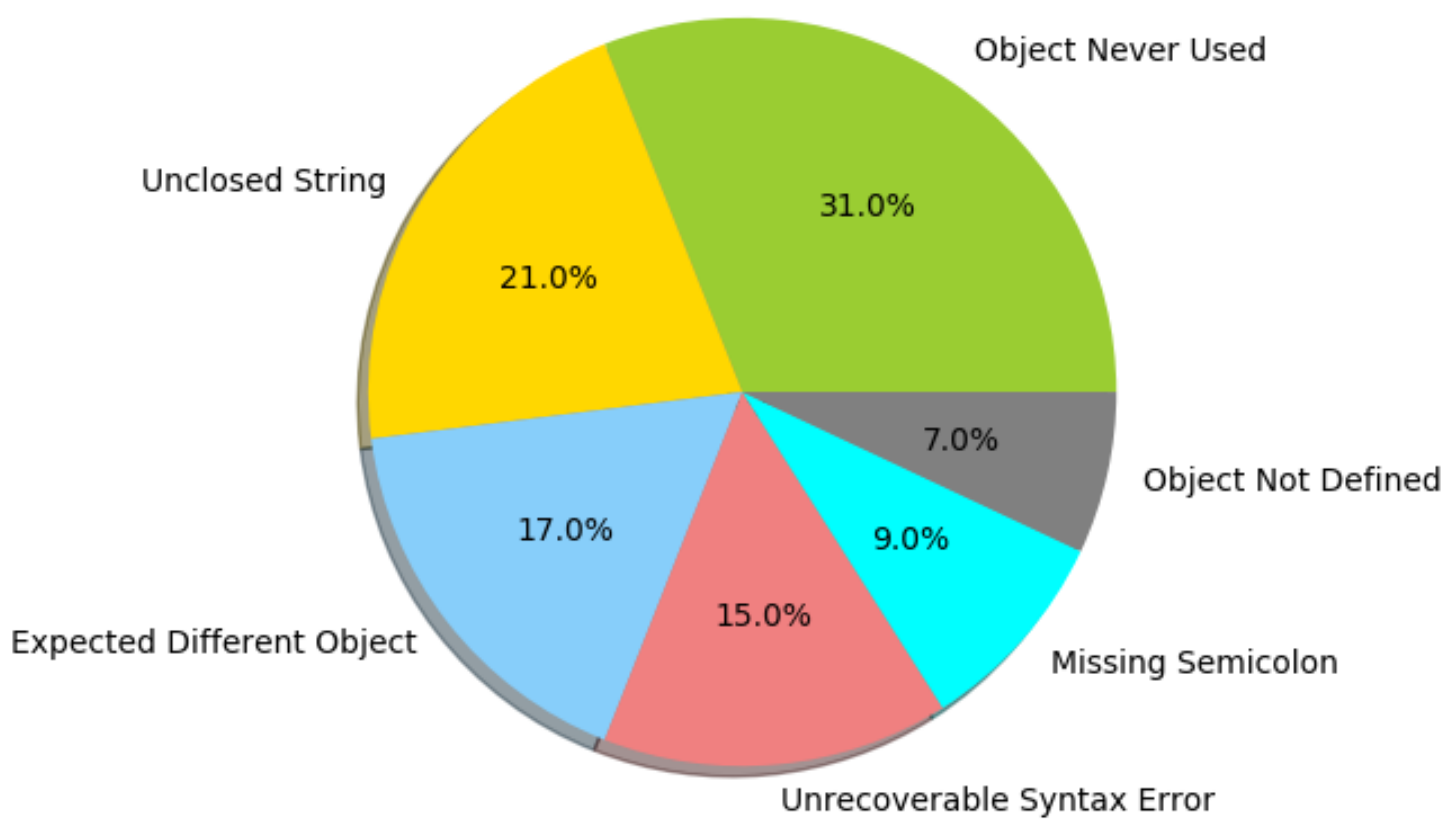

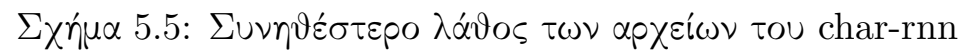

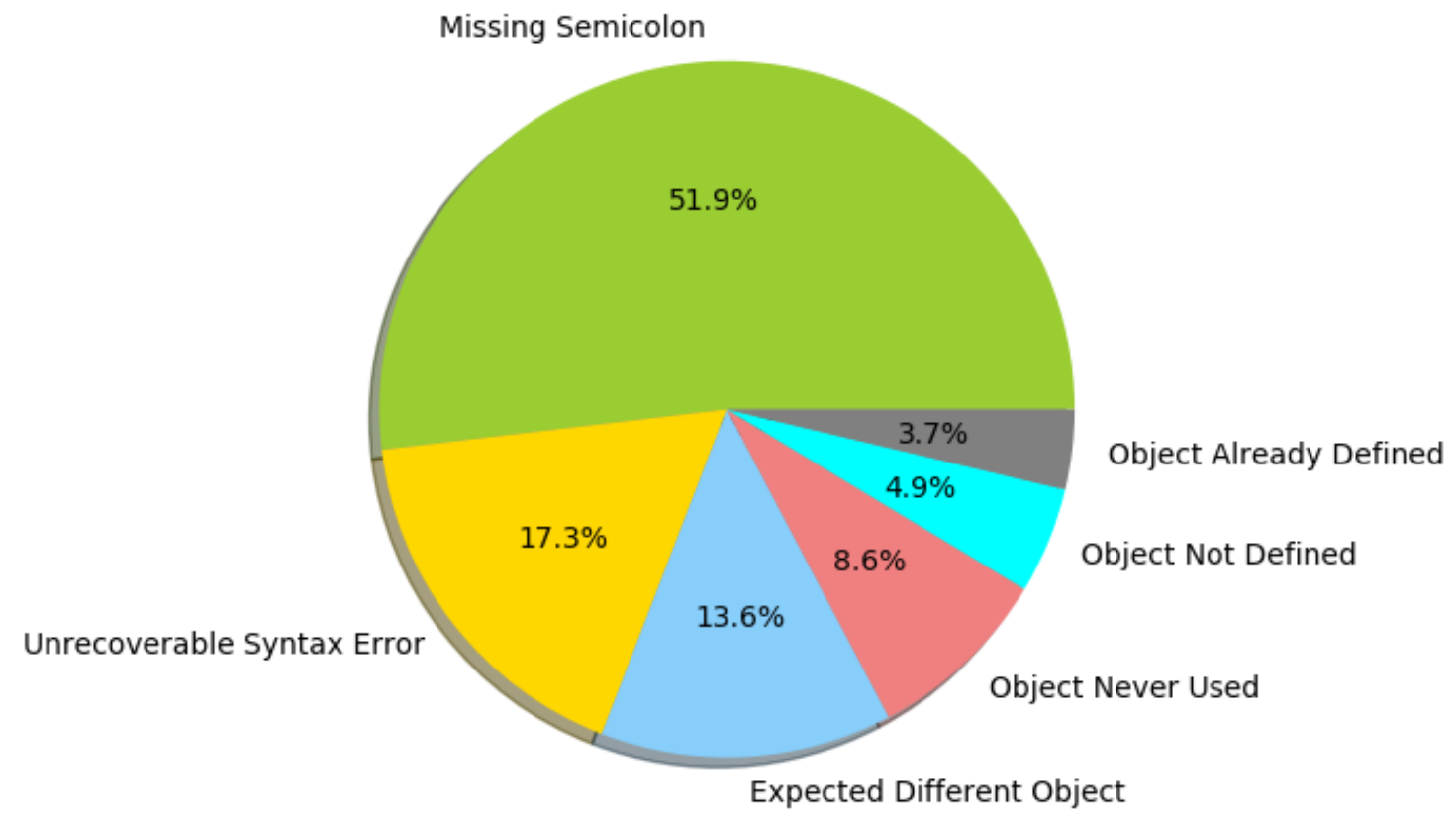

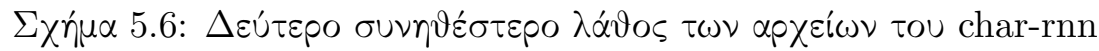

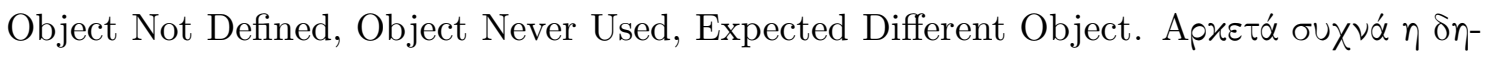

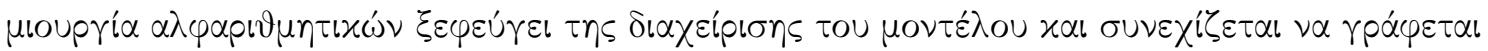

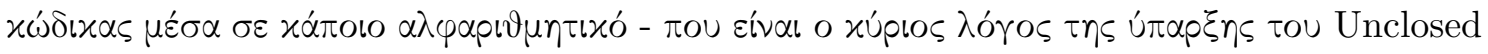
String $\sigma \varepsilon \sigma u \nu \delta \cup \alpha \sigma \mu o ́ ~ \mu \varepsilon$ to Missing Semicolon. 


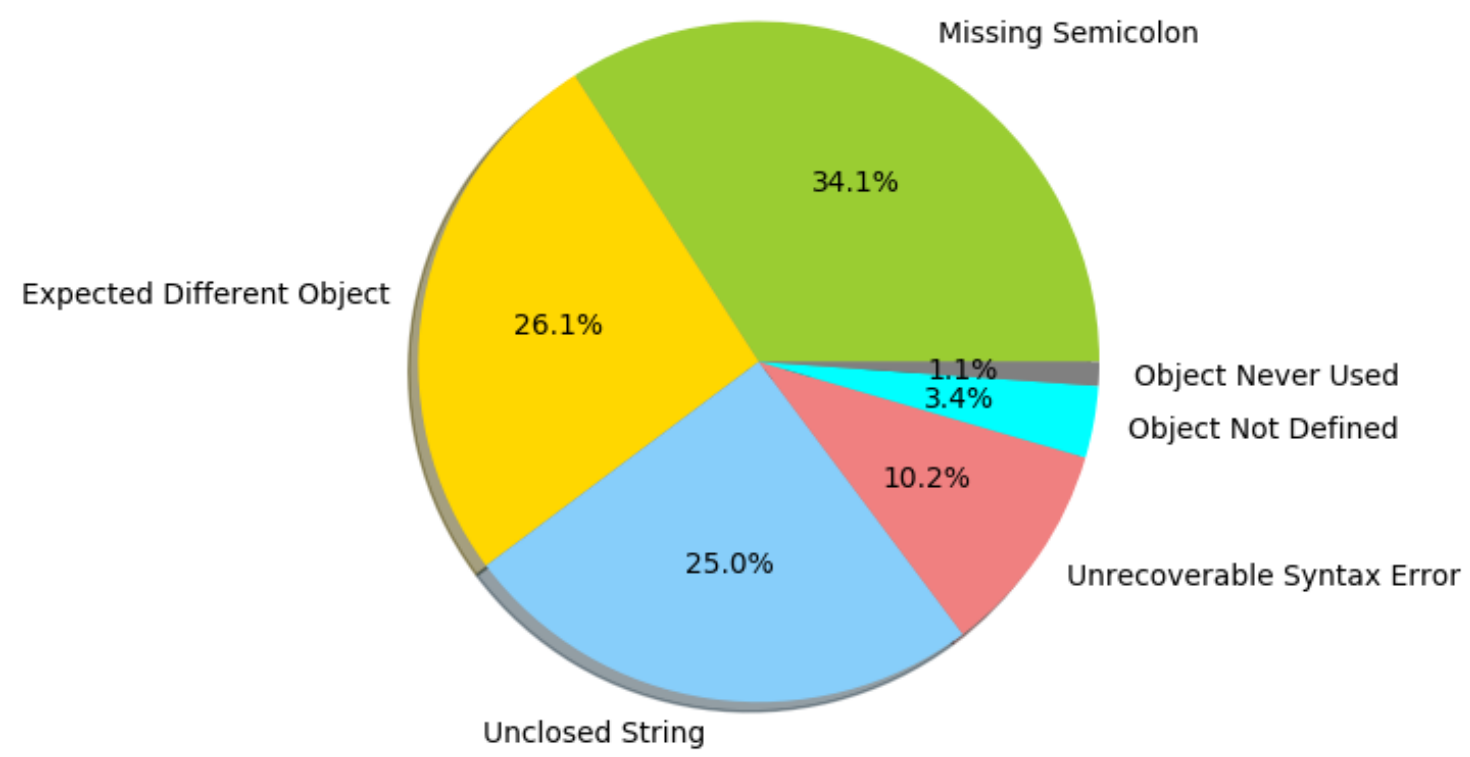

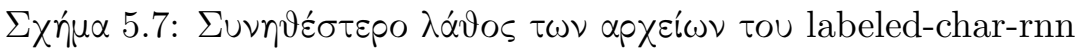

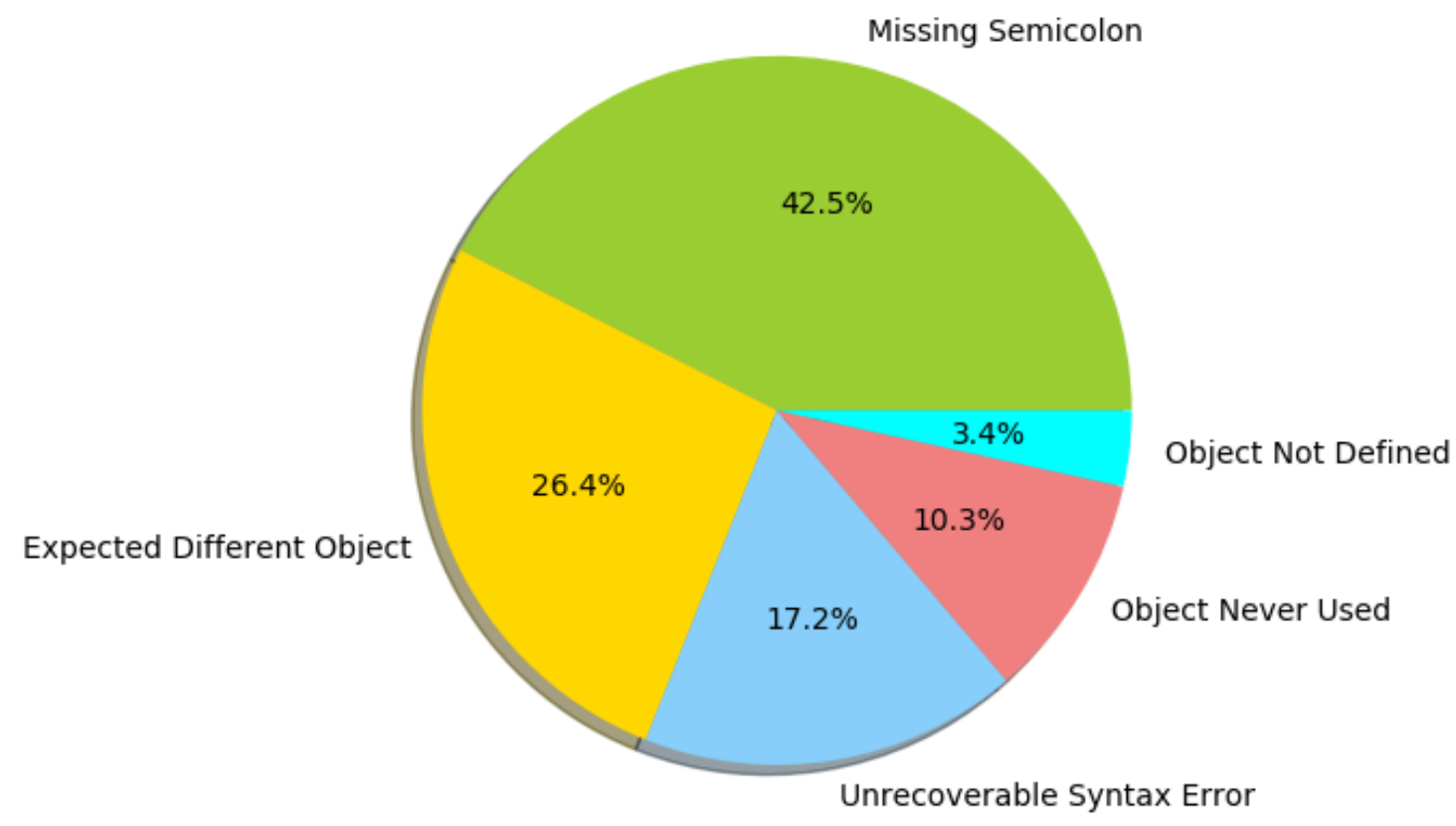

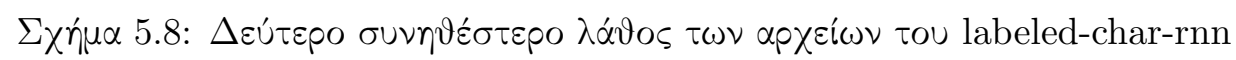

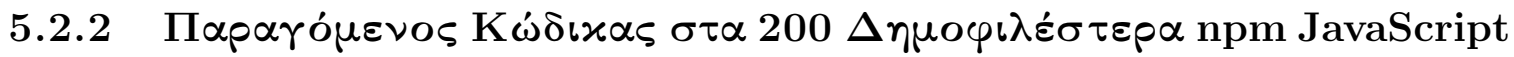 Projects}

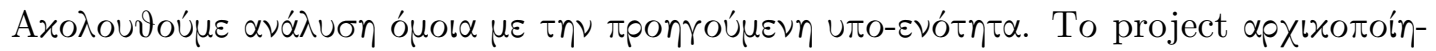

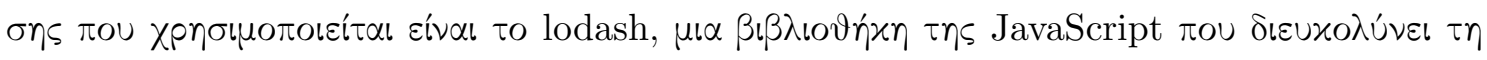

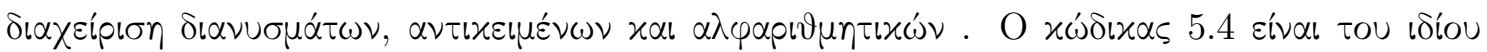

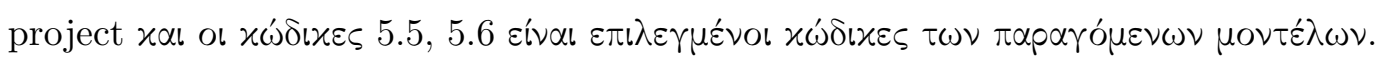




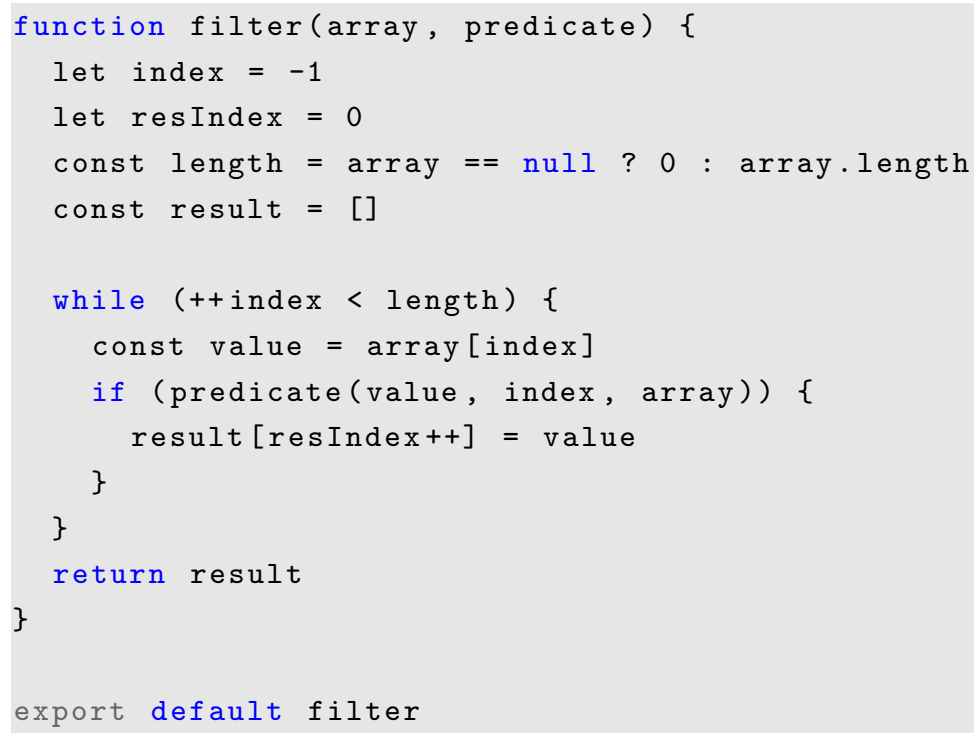

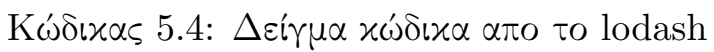

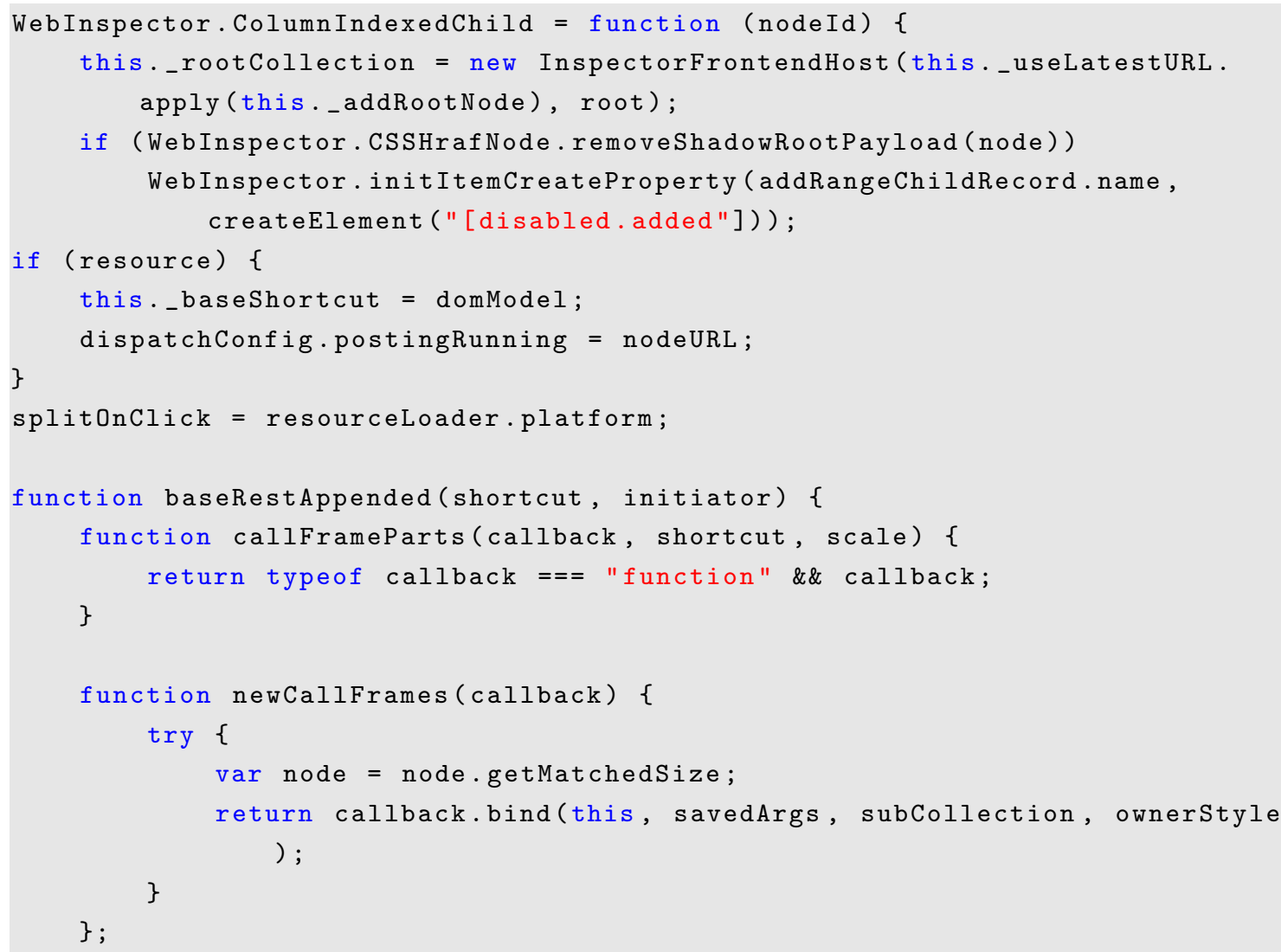

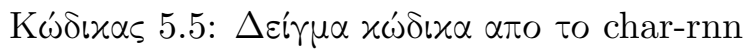




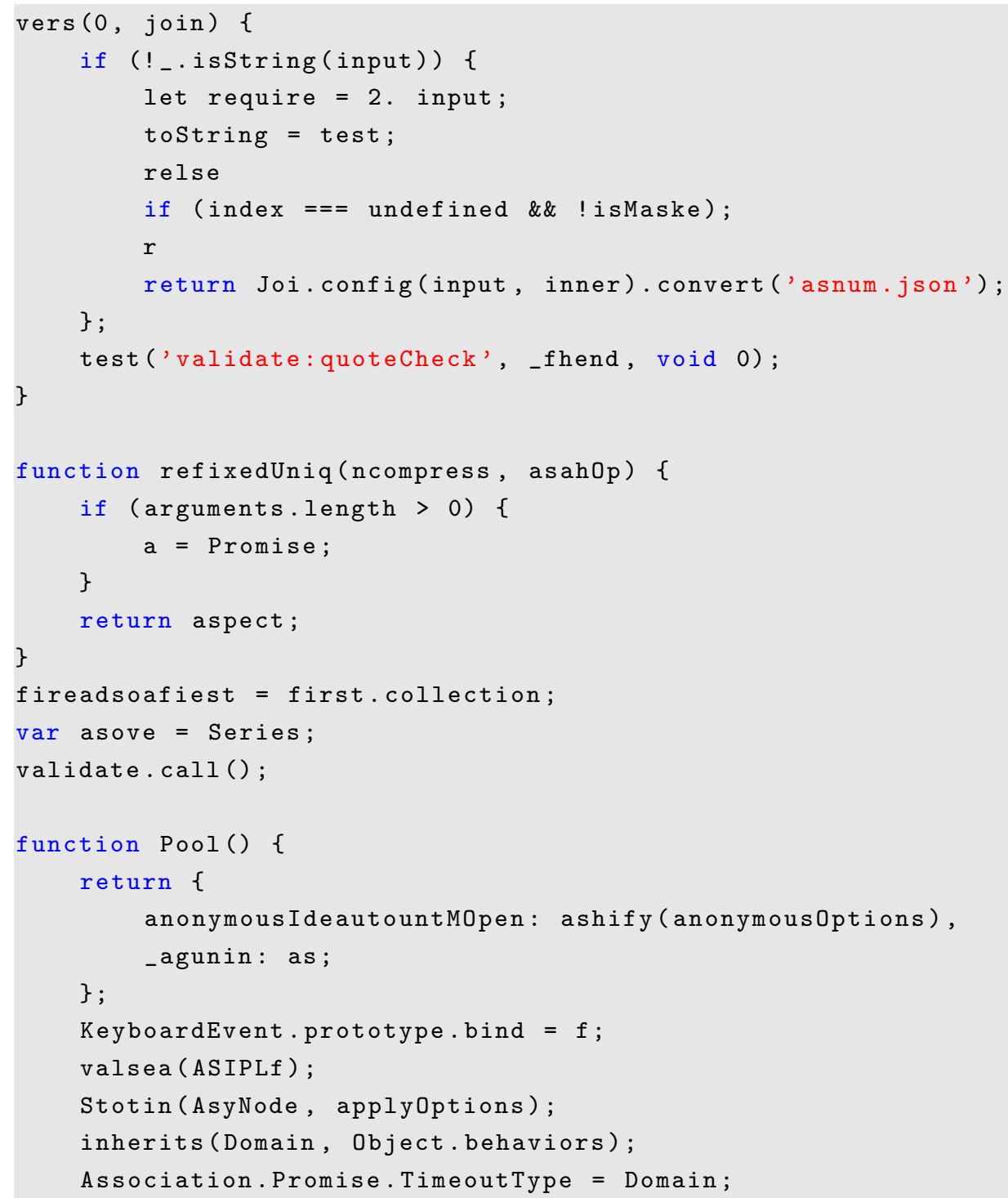

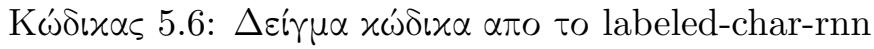

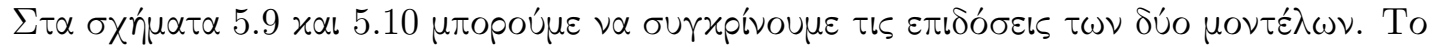

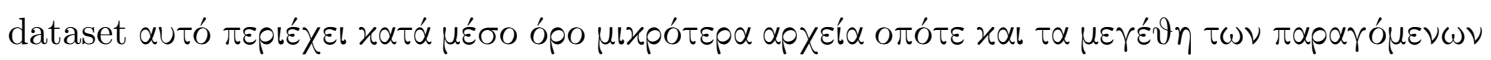

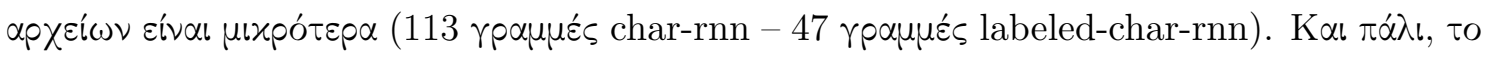

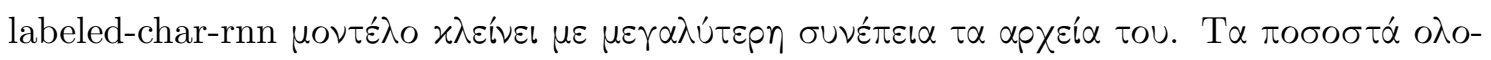

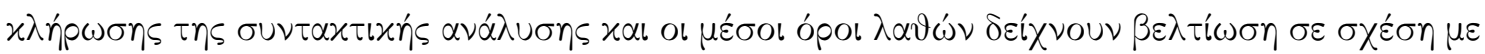

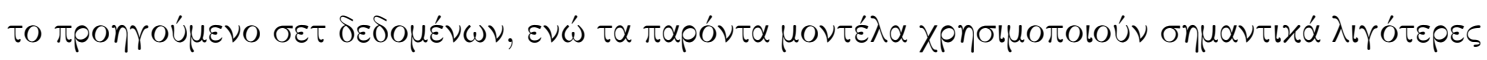

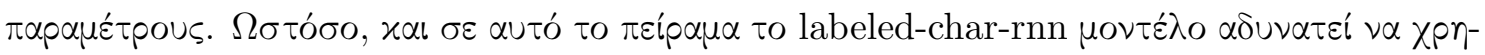

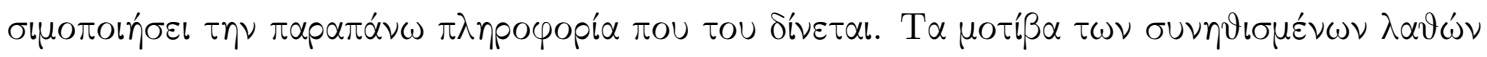

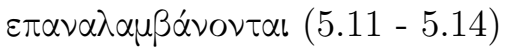




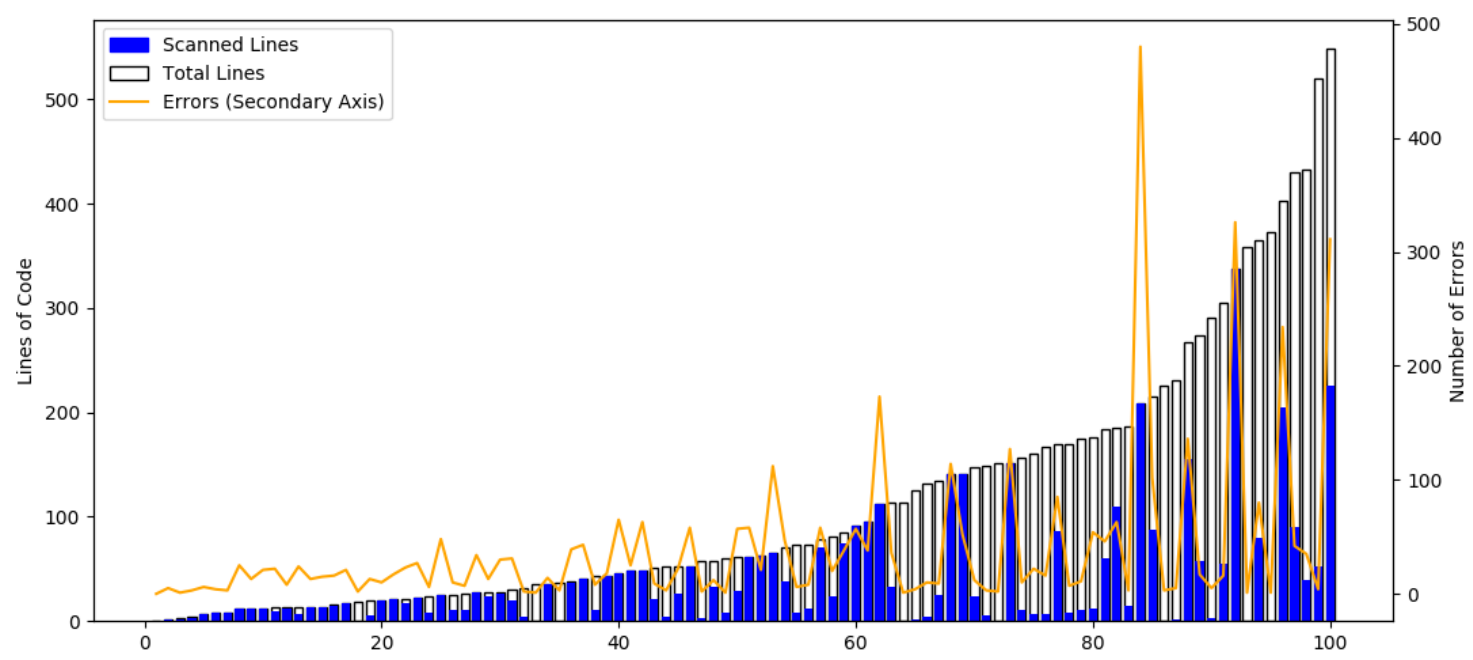

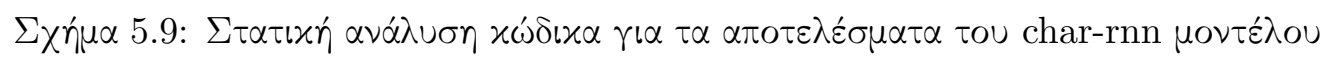

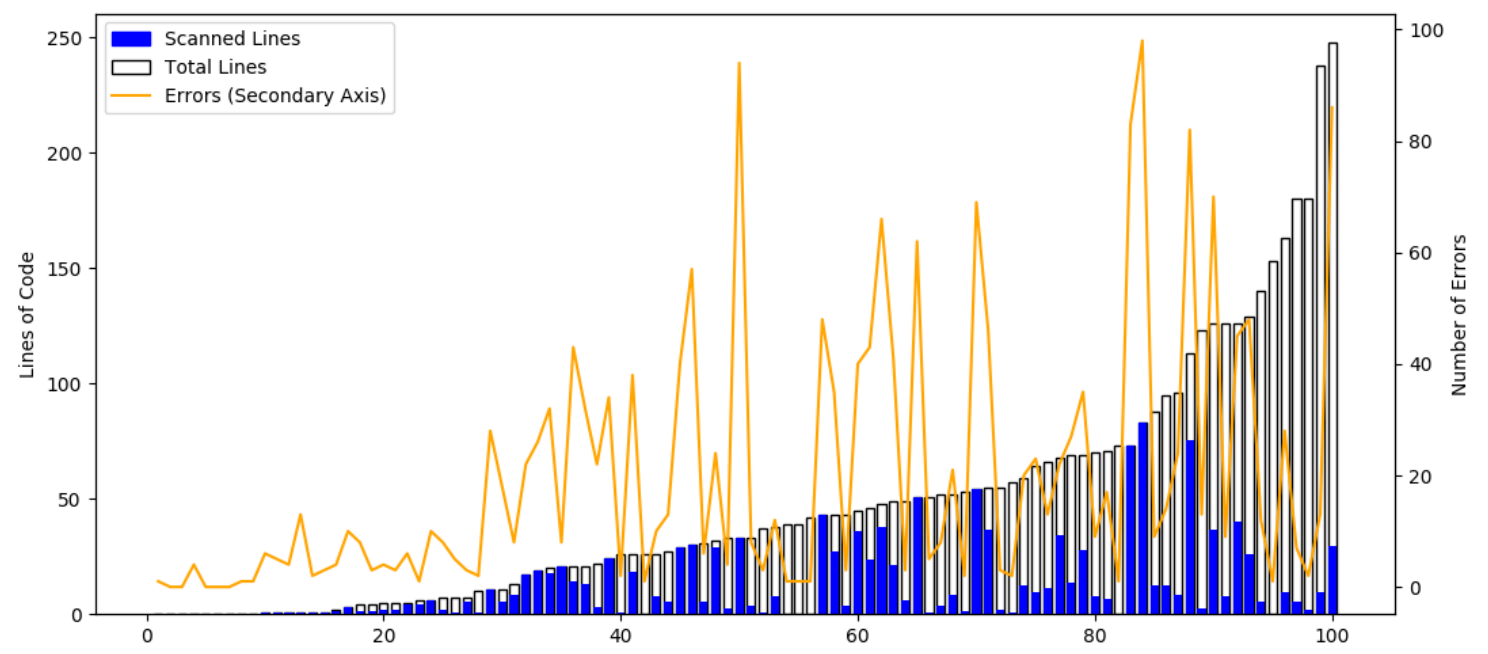

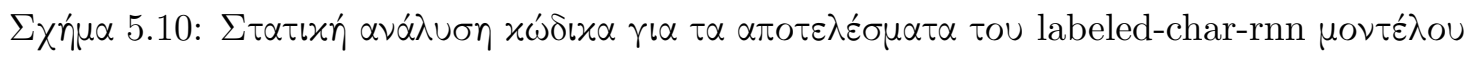




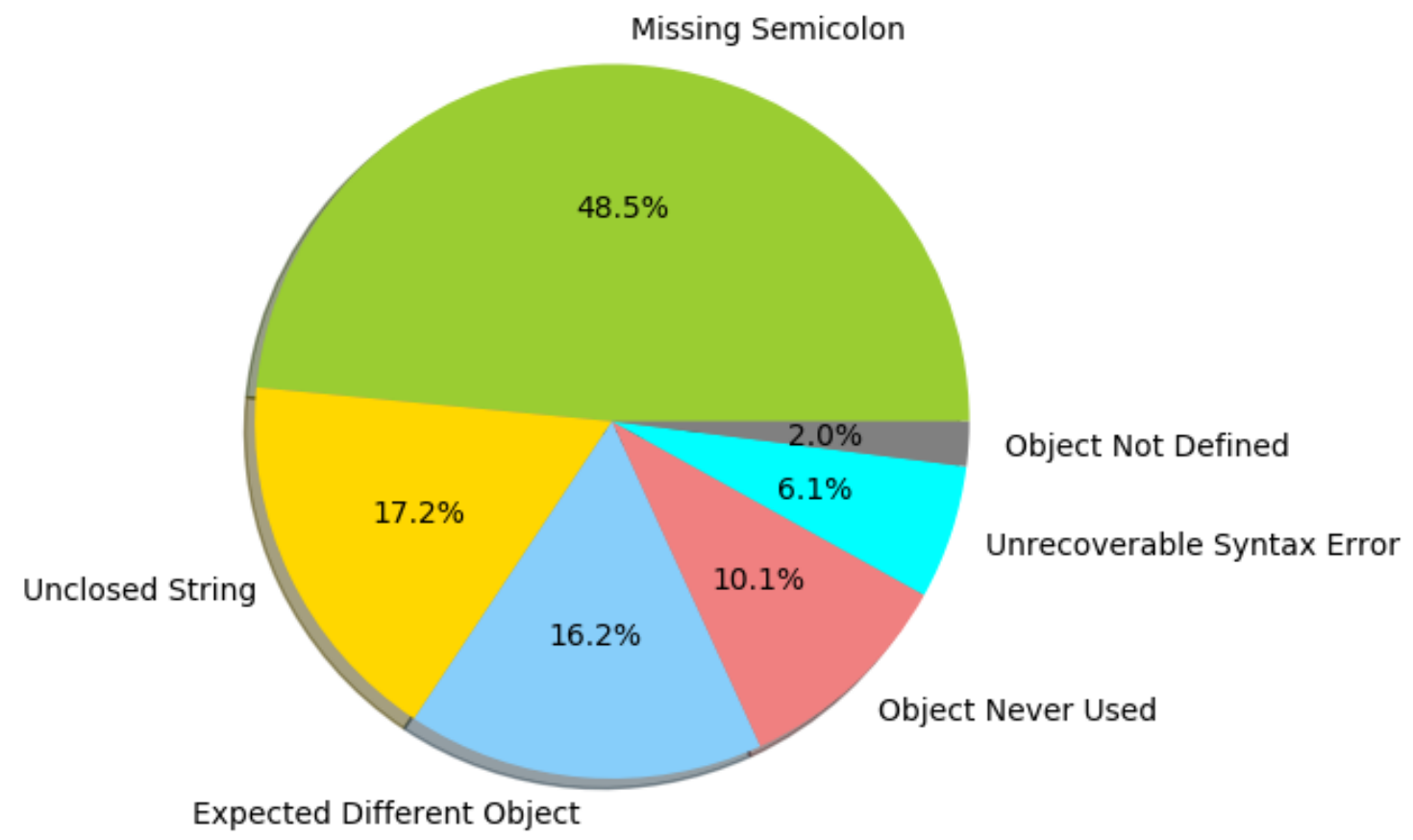

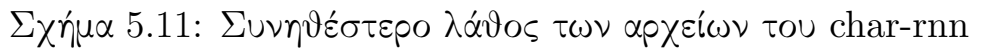

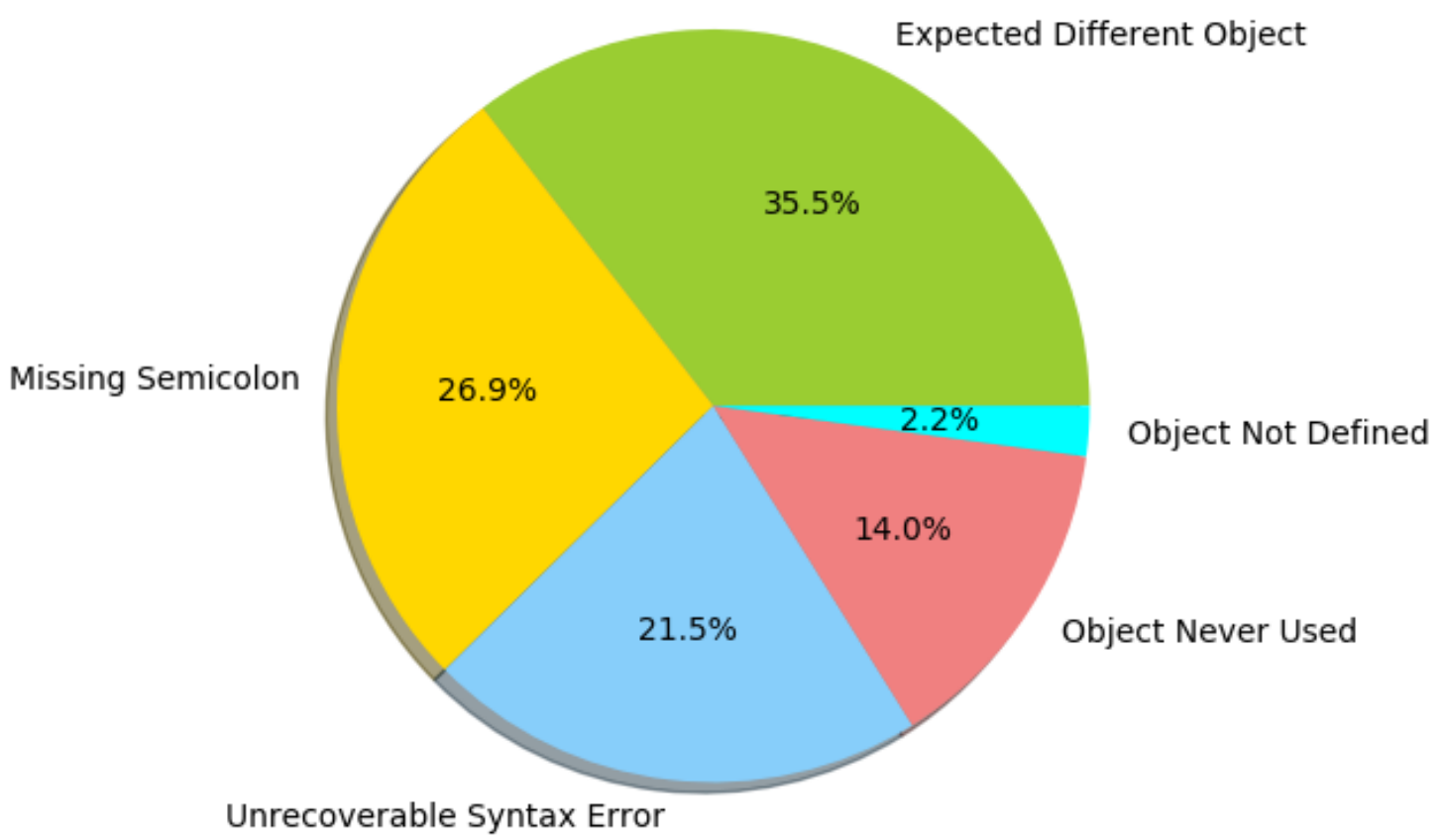

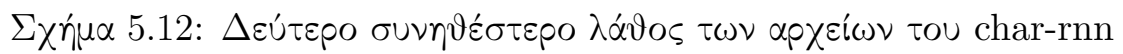




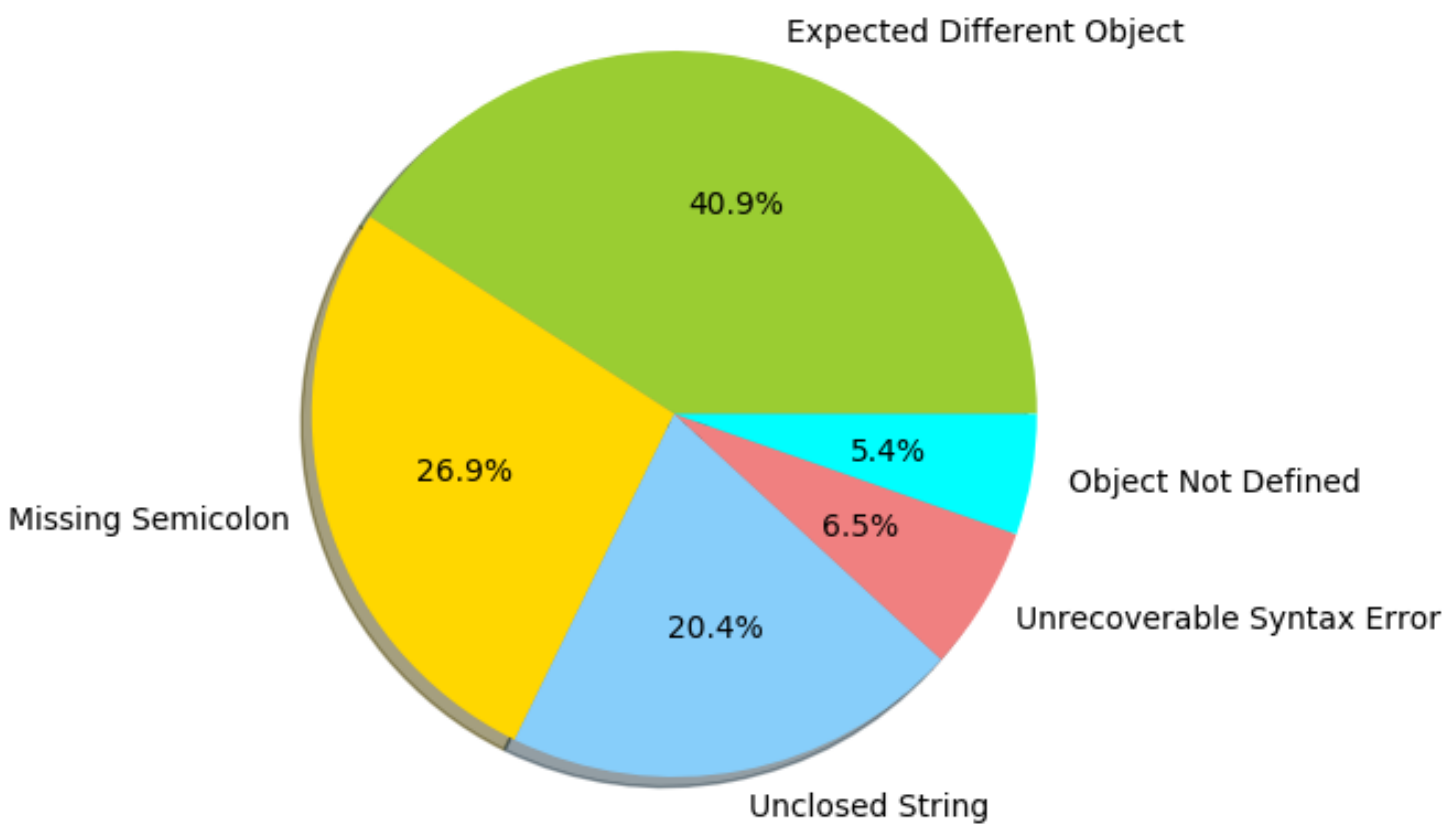

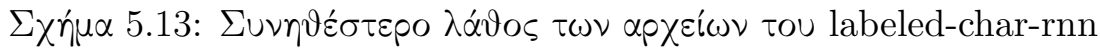

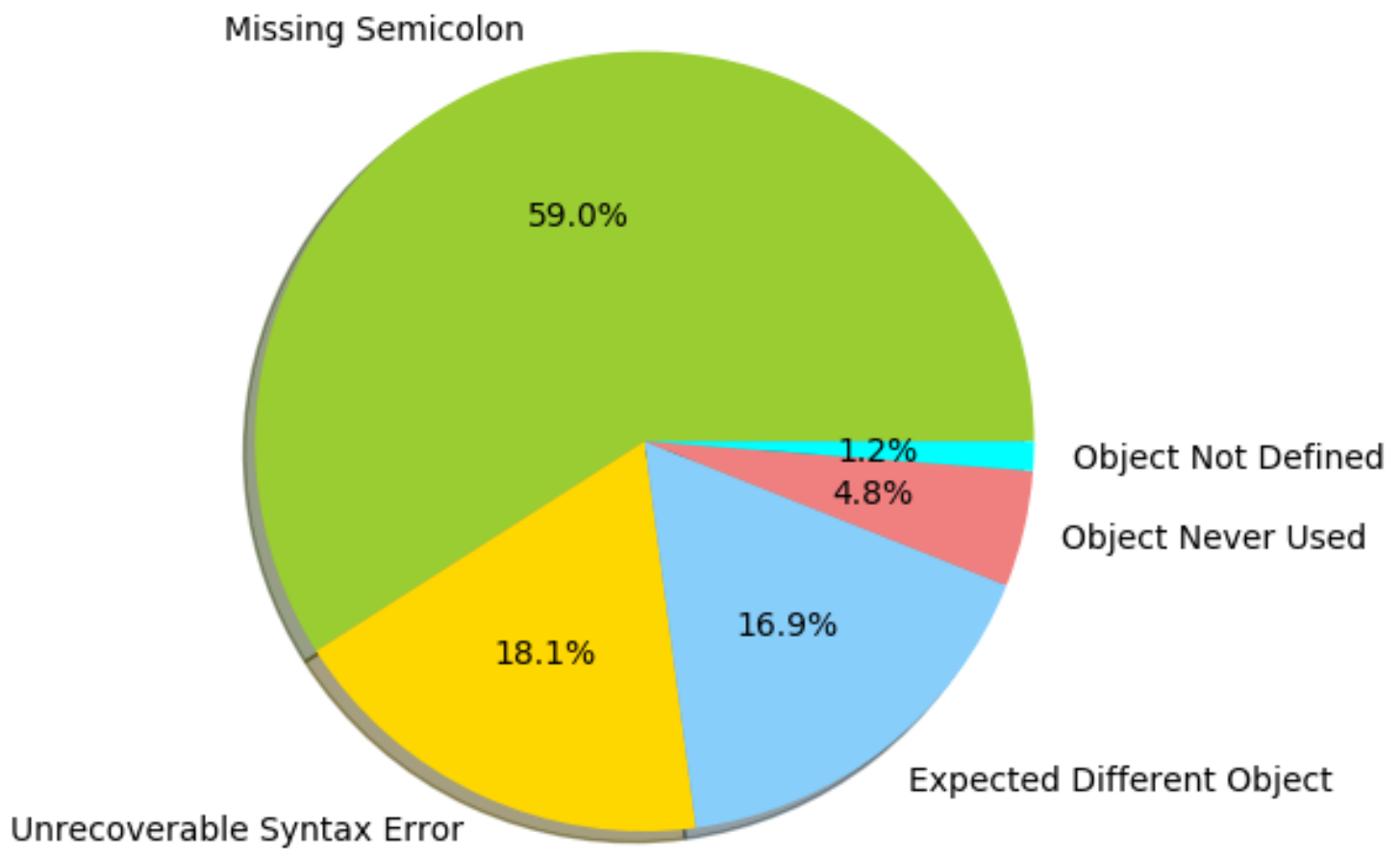

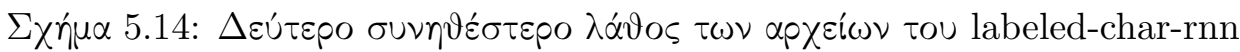




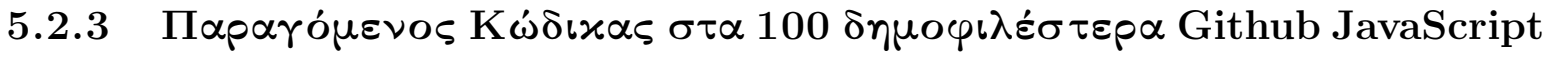

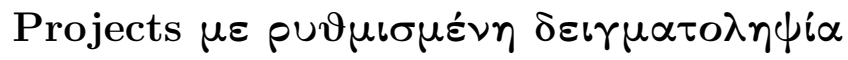

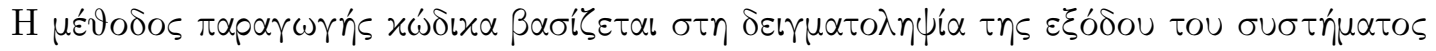

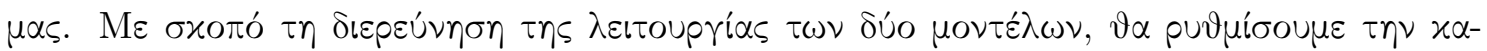

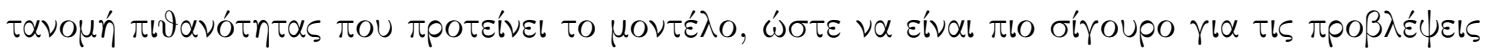

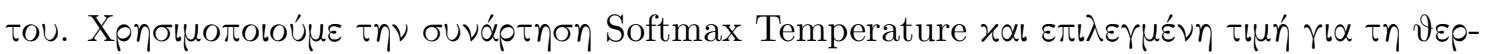

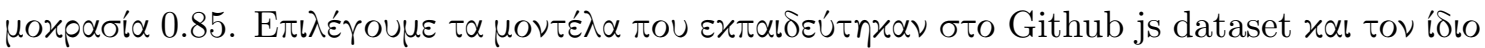

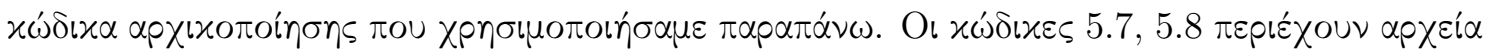

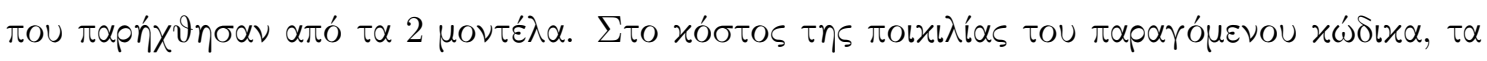

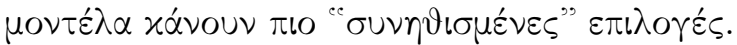

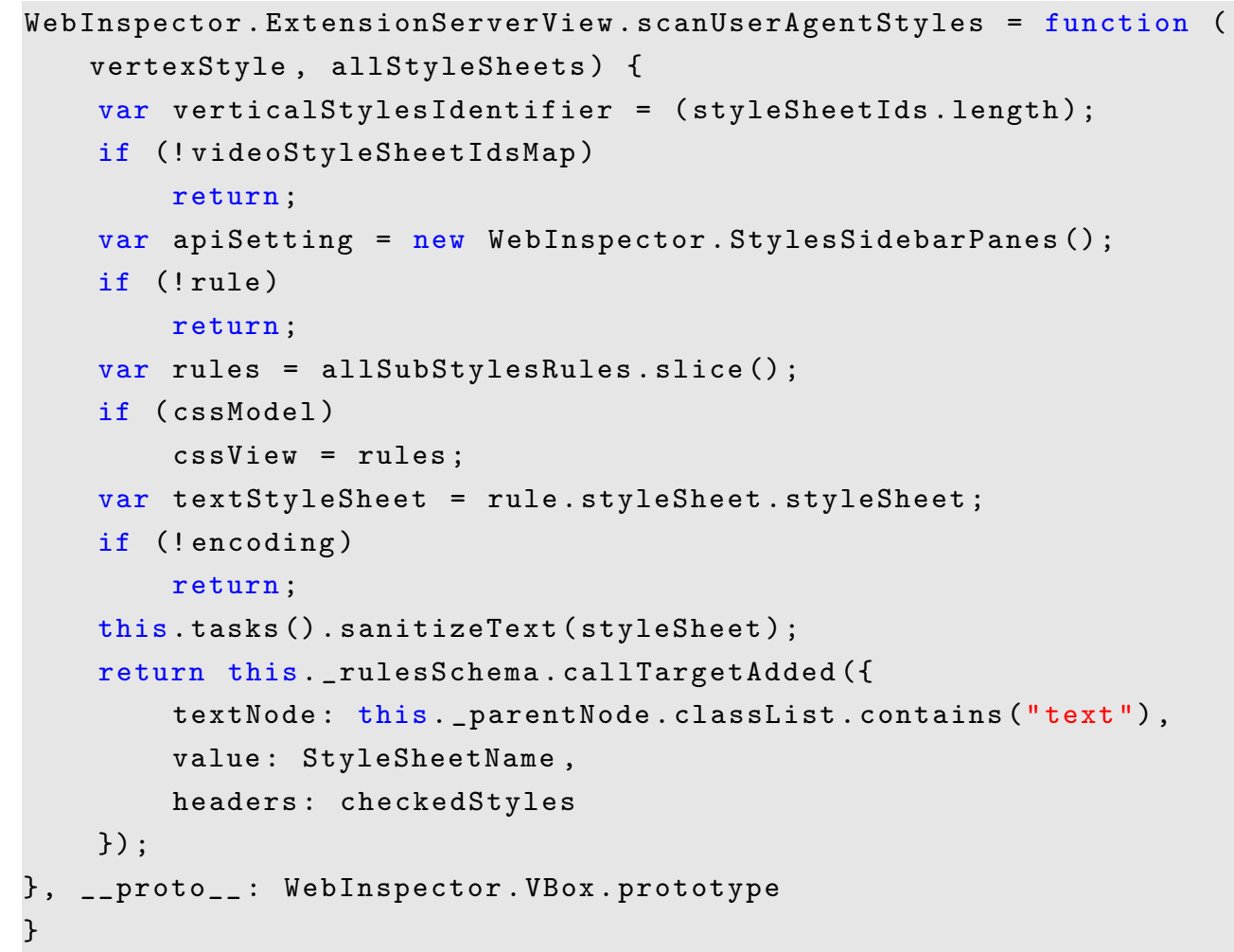

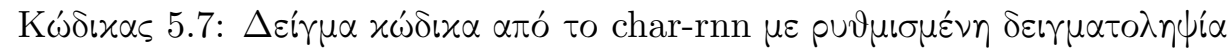




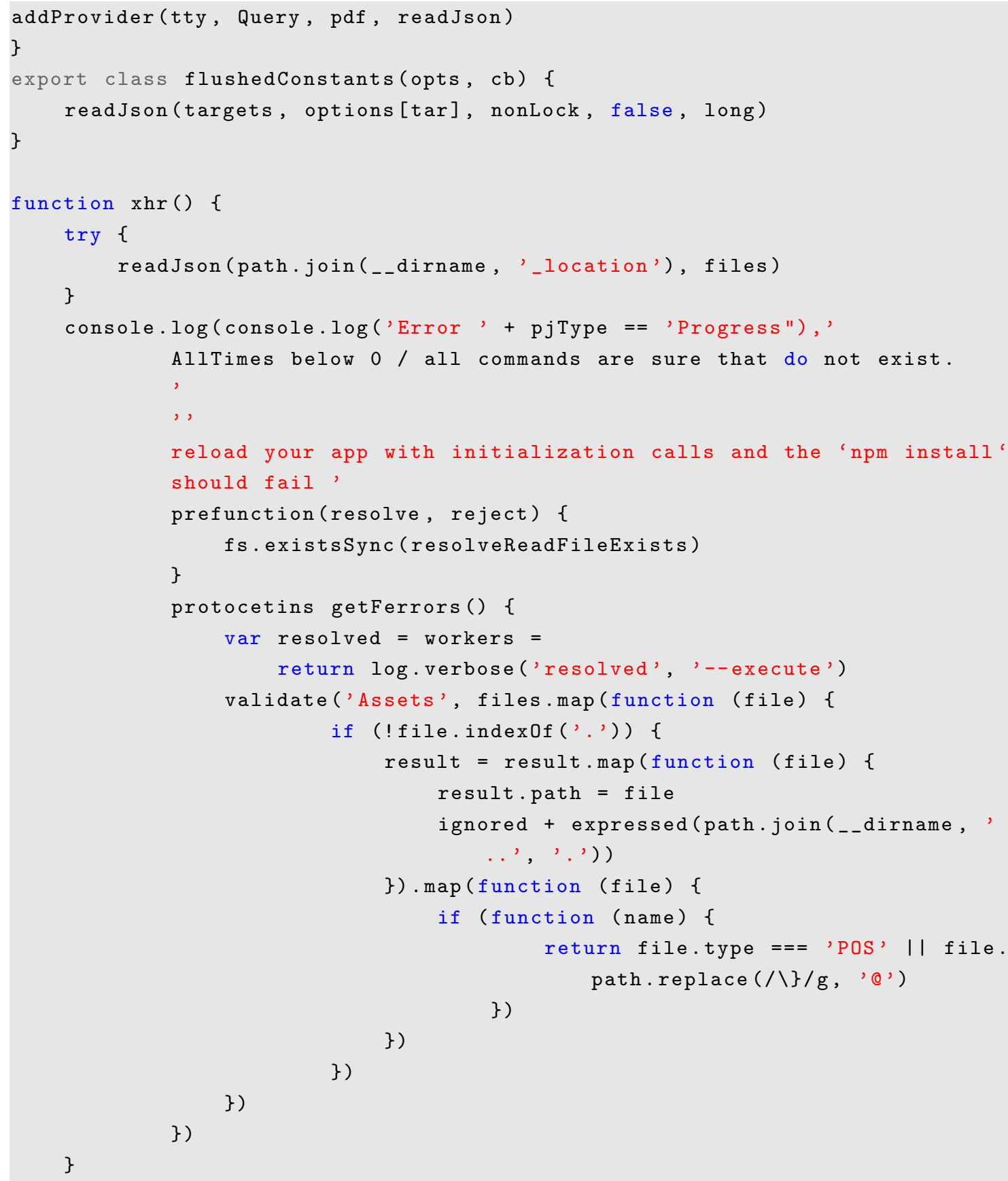

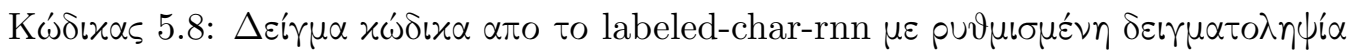

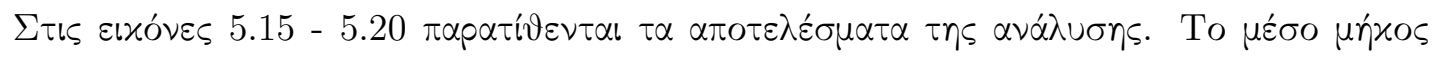

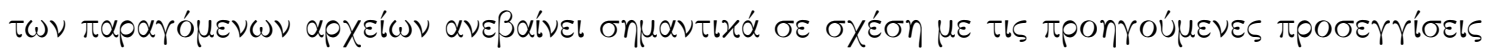

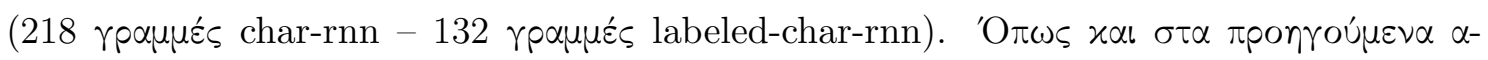

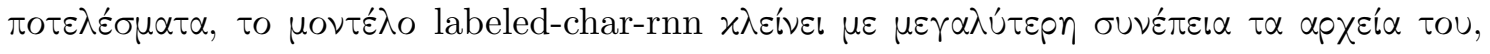

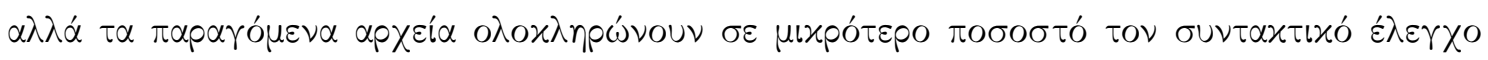

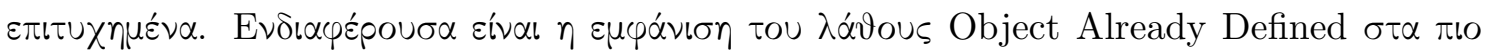

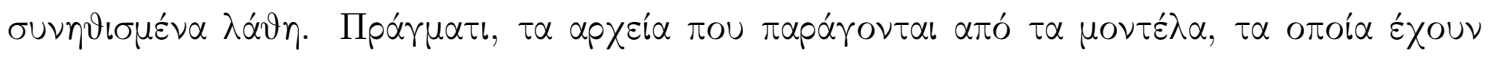

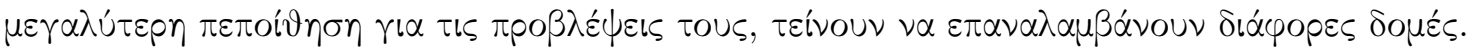




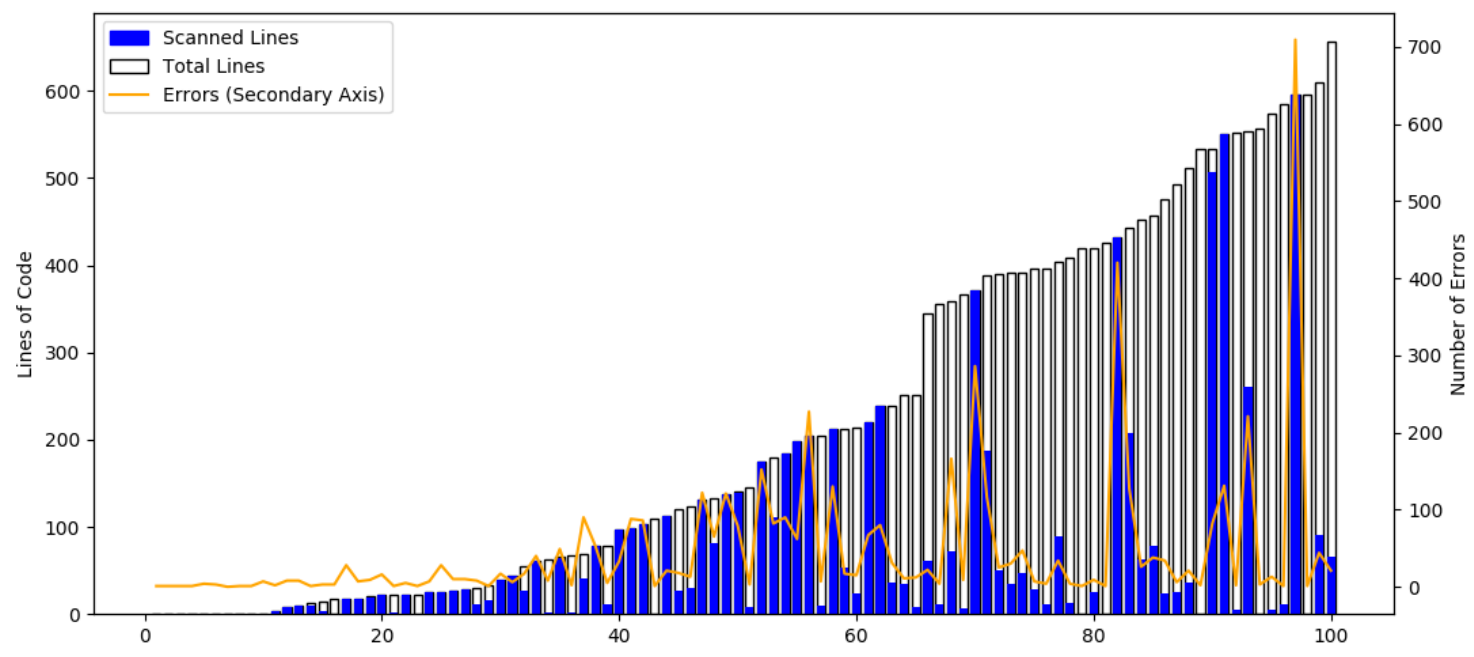

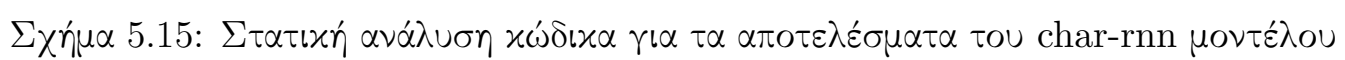

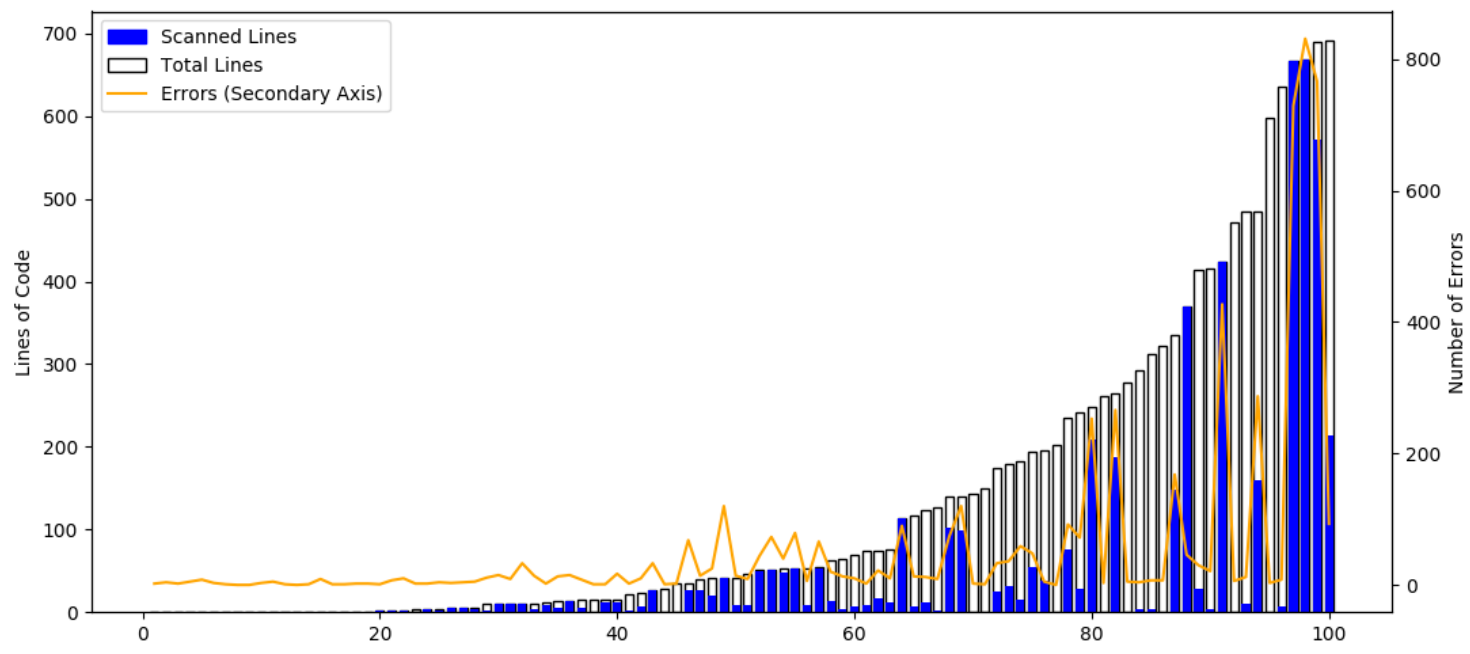

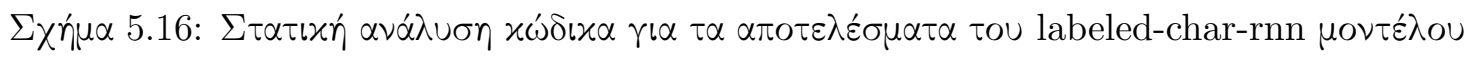




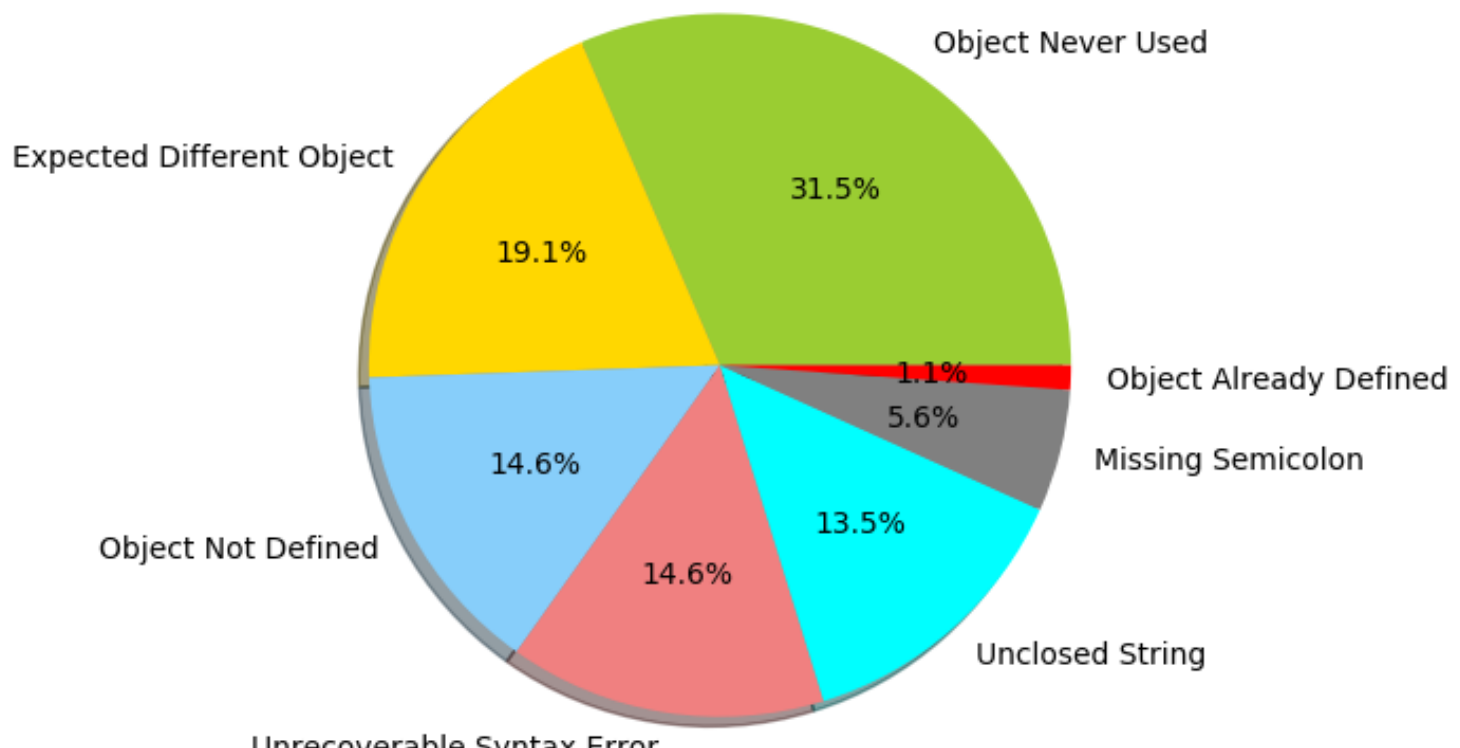

Unrecoverable Syntax Error

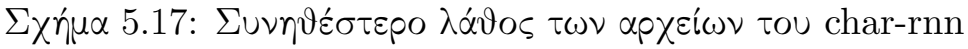

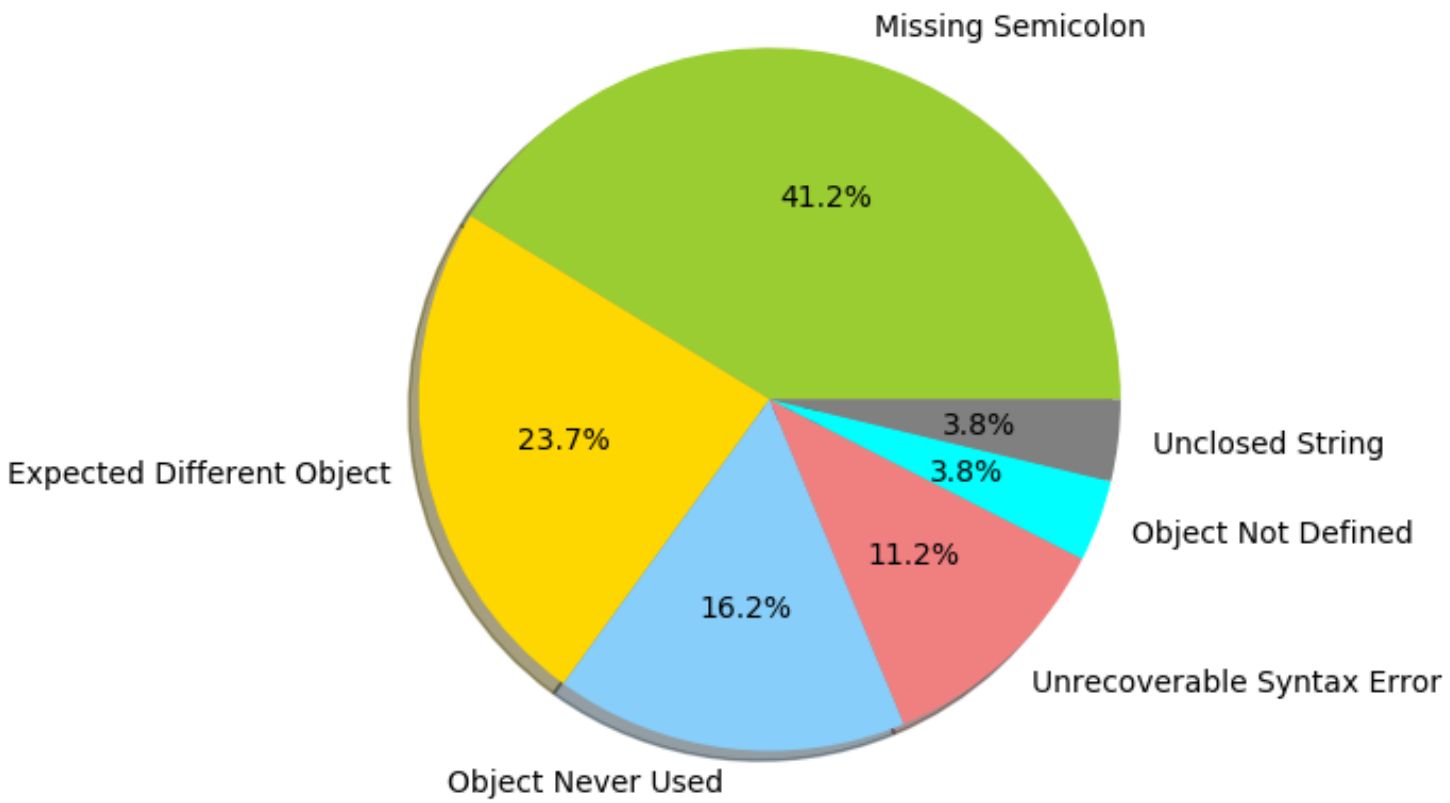

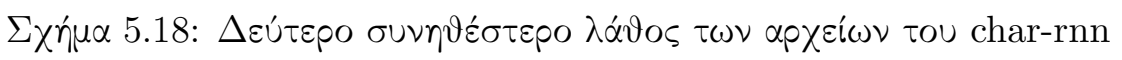




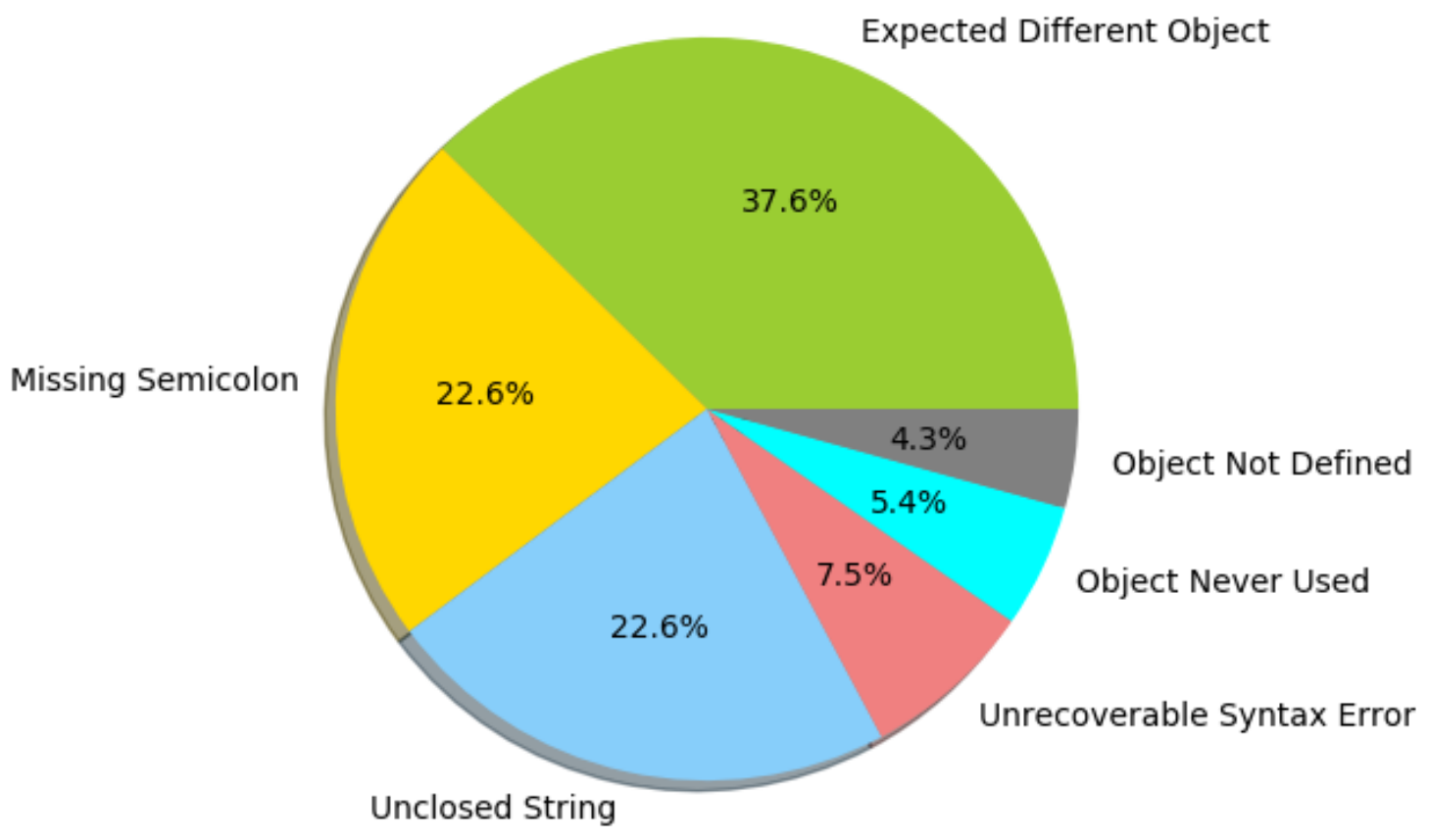

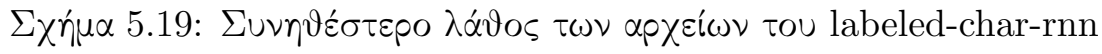

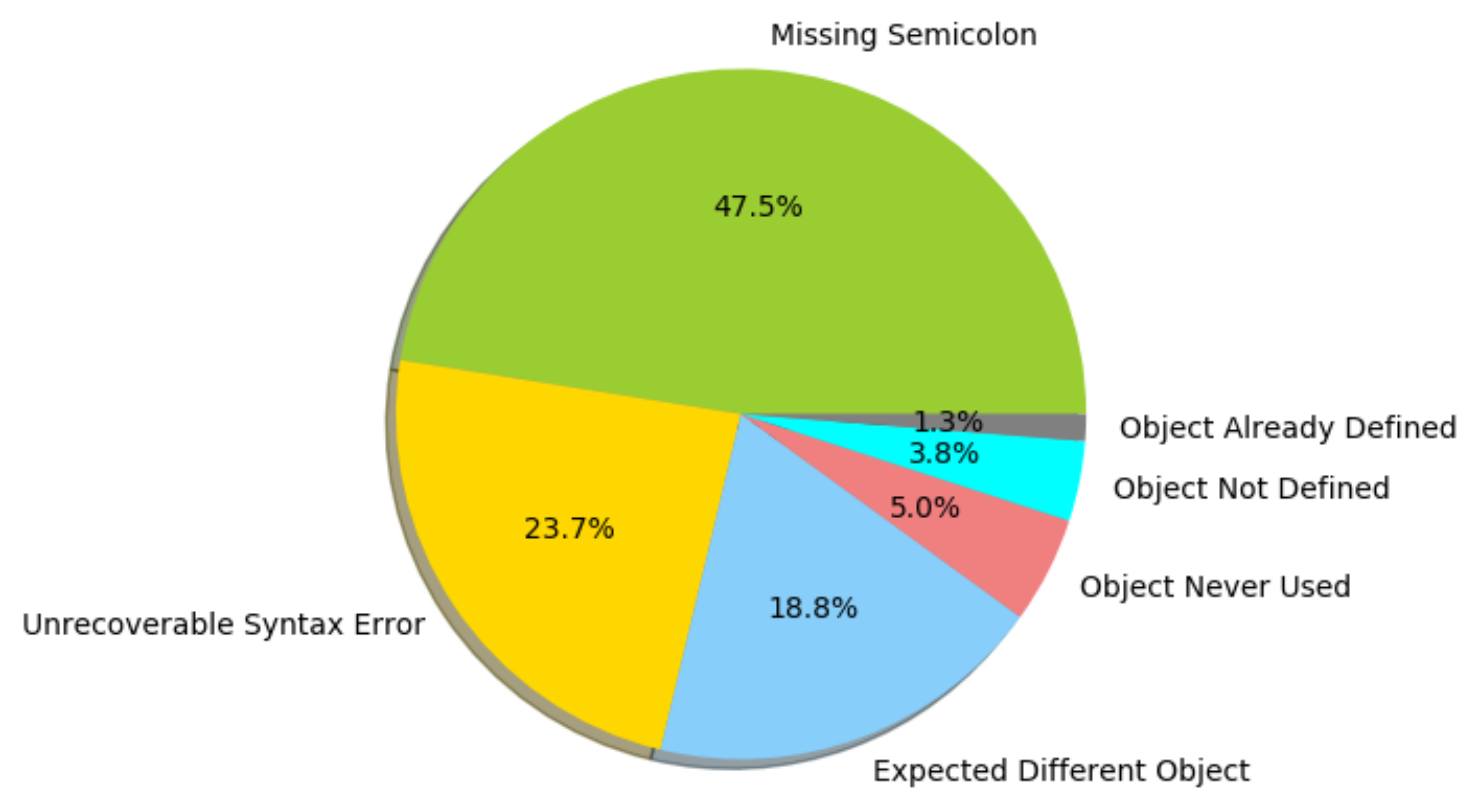

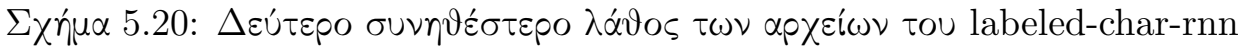




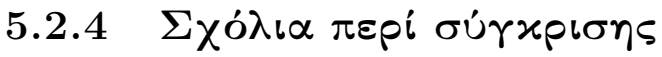

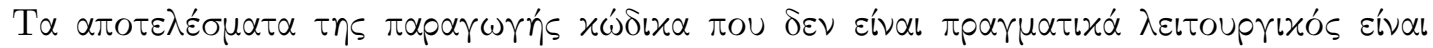

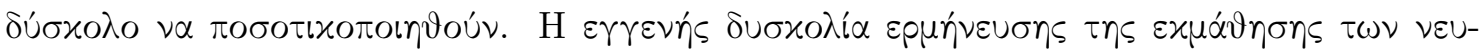

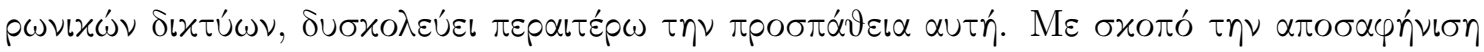

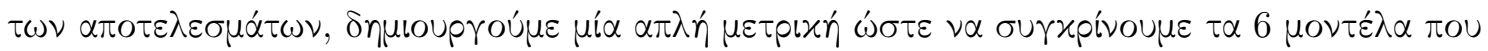
$\delta o x \mu \alpha \dot{\sigma} \sigma \tau \eta \alpha \alpha$.

$$
M=\text { Error }_{\text {avg }} /\left(\text { Lines }_{\text {avg }} * \% \text { Scanned }_{\text {avg }}\right)
$$

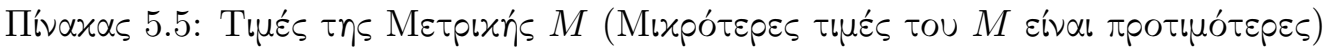

\begin{tabular}{|l|l|l|}
\hline & char-rnn & labeled-char-rnn \\
\hline Github & 0.61 & 0.86 \\
\hline NPM & 0.64 & 0.77 \\
\hline Github Temperature & 0.38 & 0.52 \\
\hline
\end{tabular}

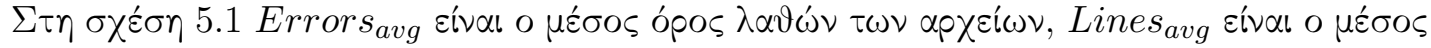

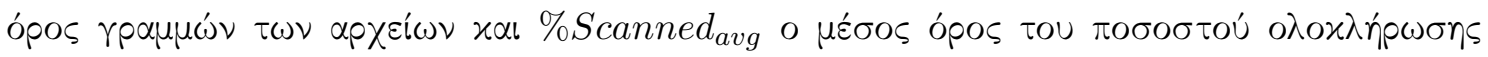

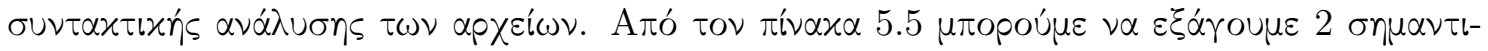

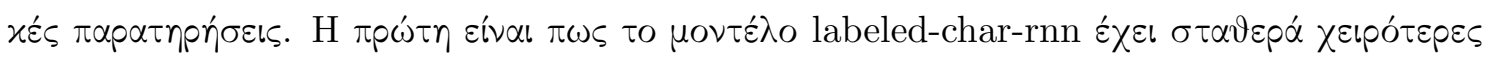

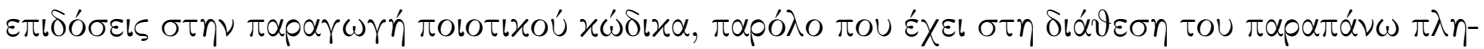

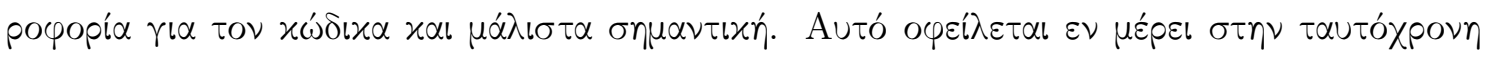

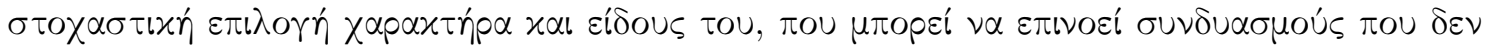

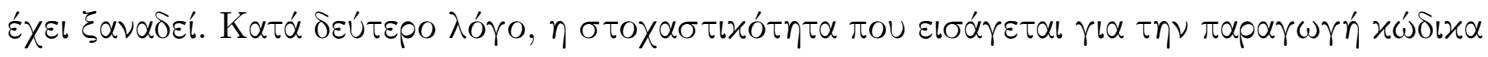

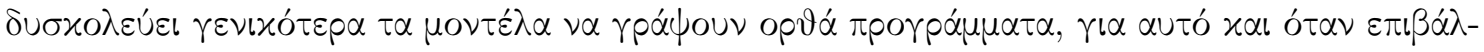

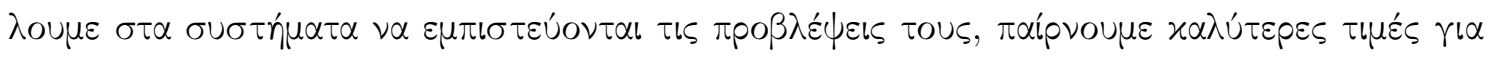

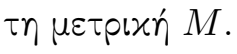




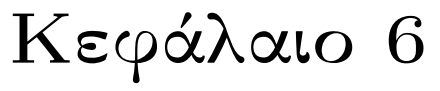

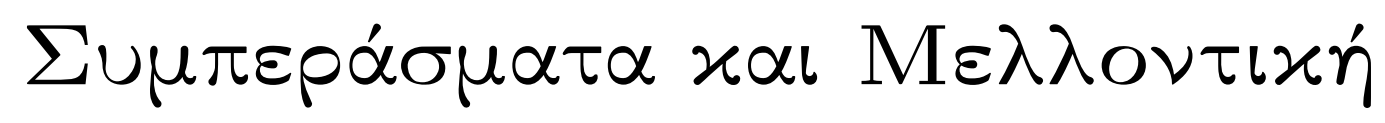

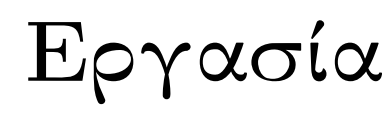

\section{$6.1 \quad \Sigma u \mu \pi \varepsilon \rho \alpha ́ \sigma \mu \alpha \tau \alpha$}

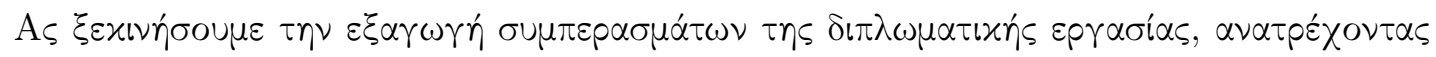

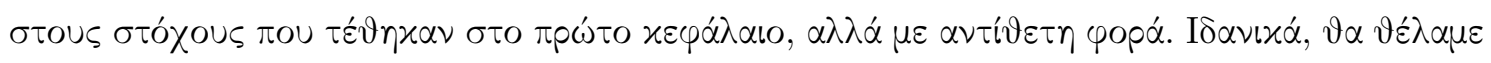

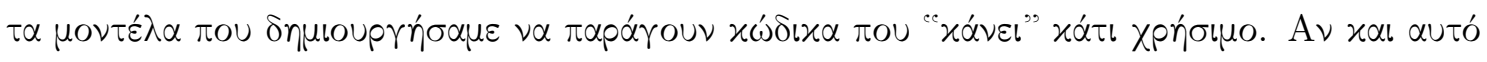

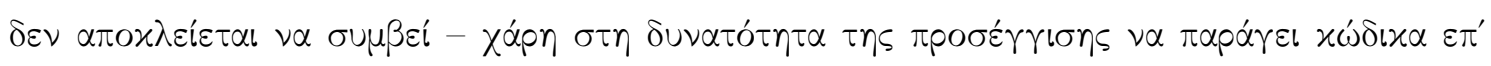

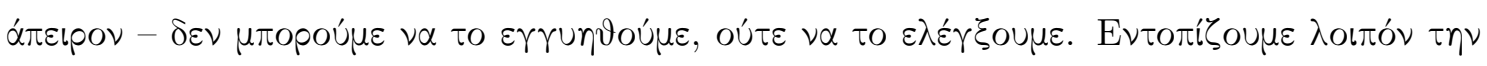

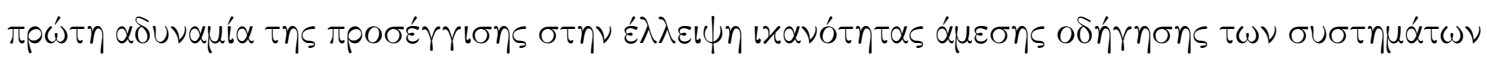

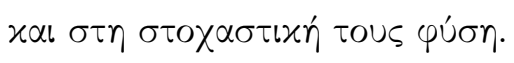

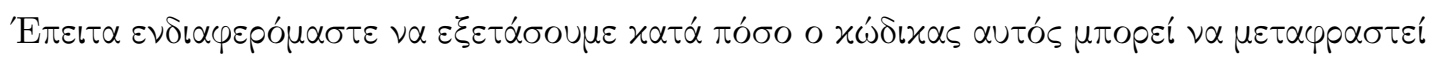

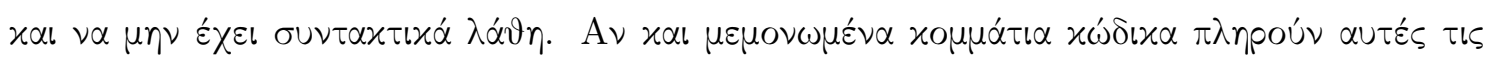

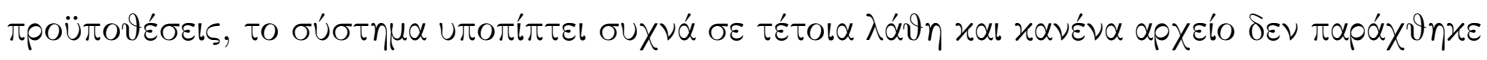

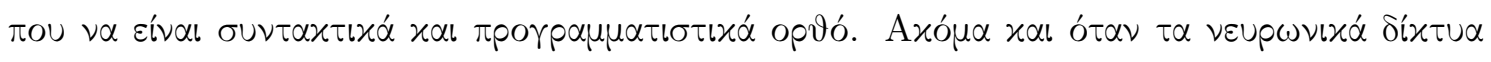

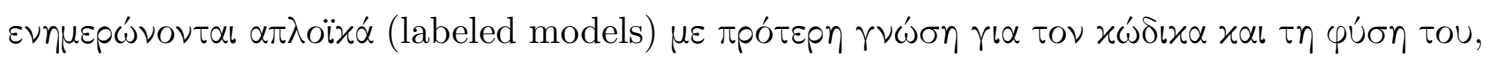

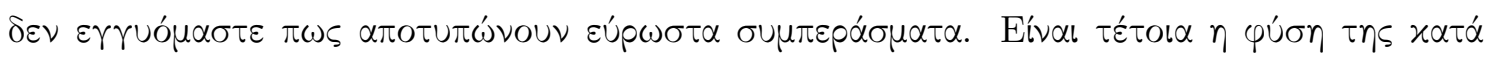

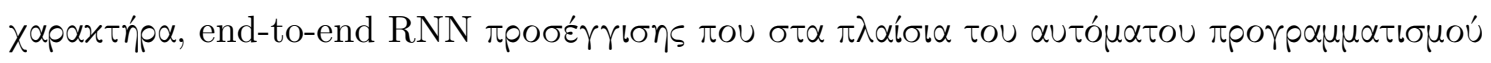

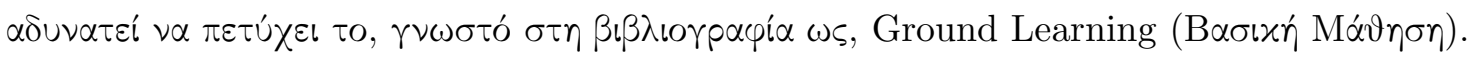

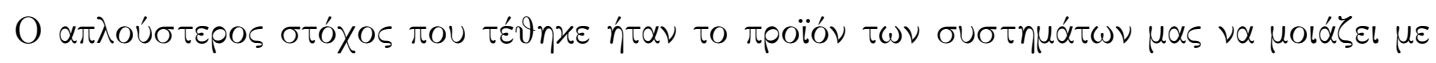

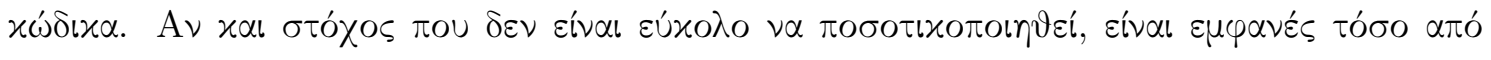

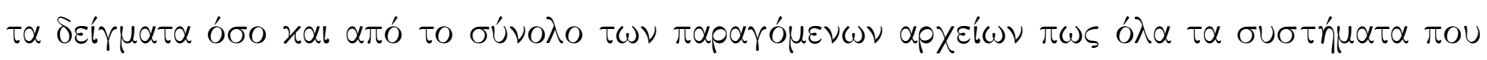

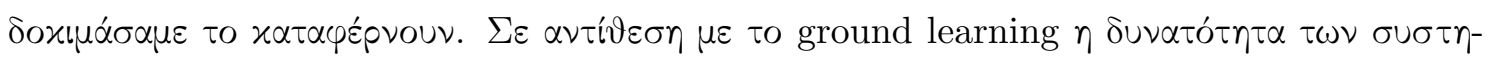

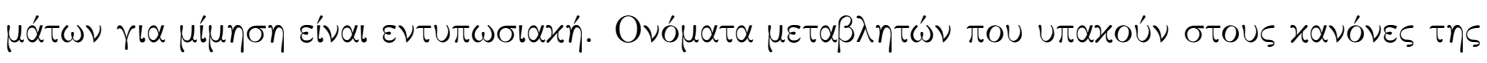

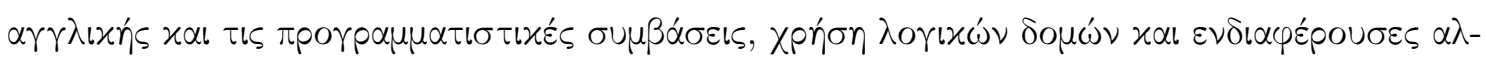

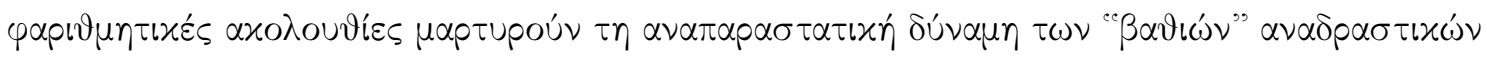

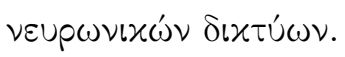

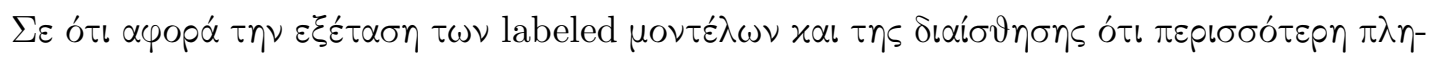

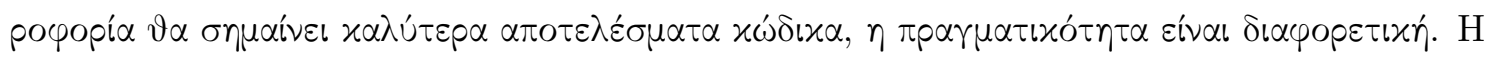




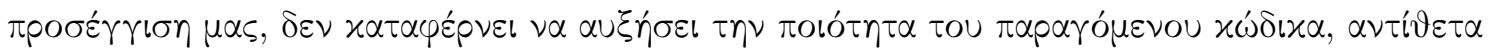

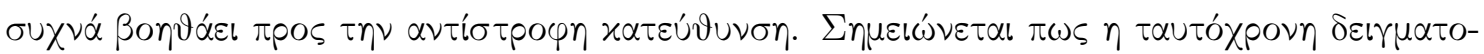

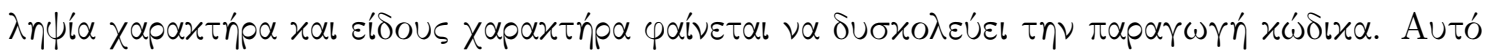

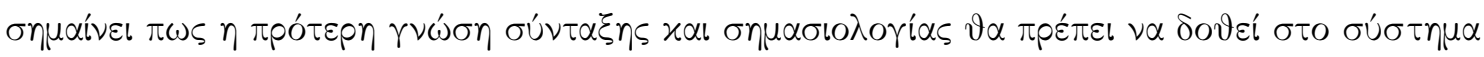

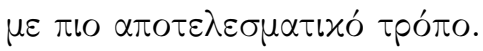

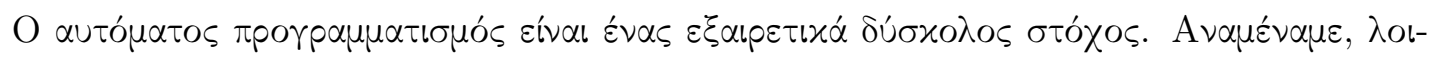

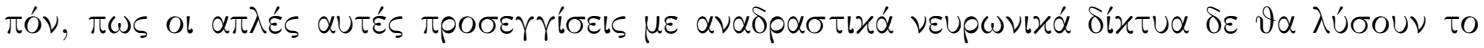

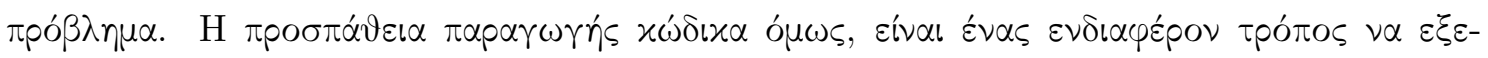

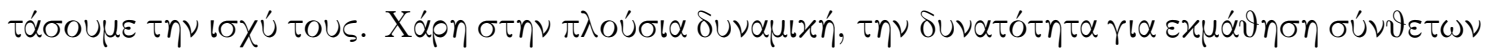

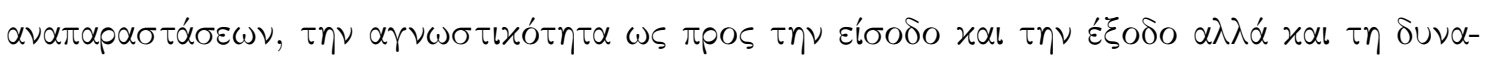

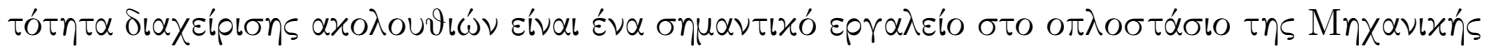
Máłฑons.

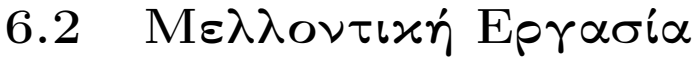

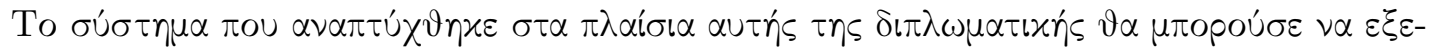

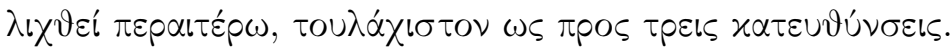

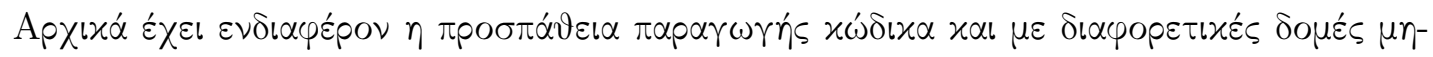

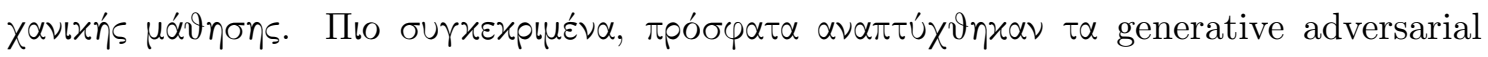

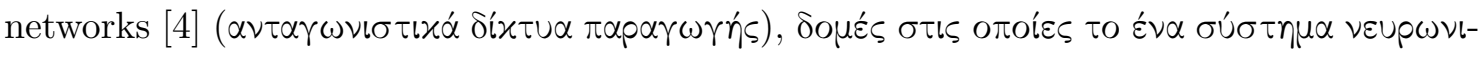

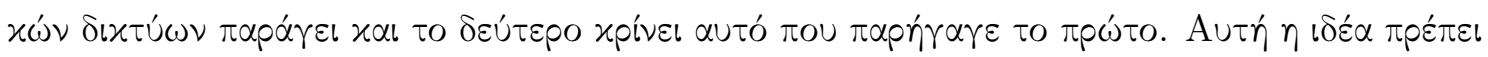

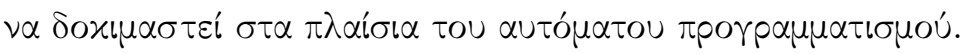

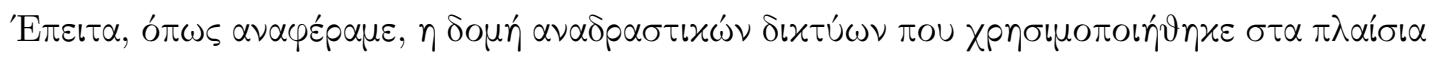

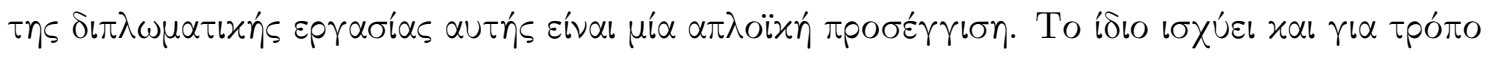

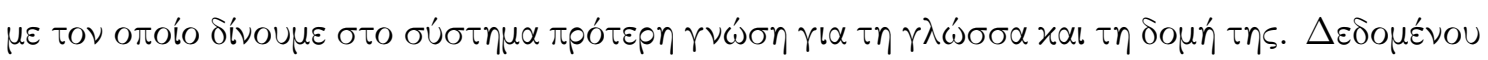

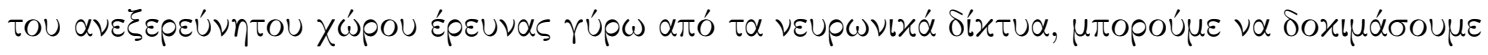

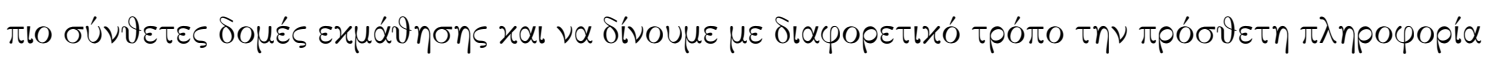

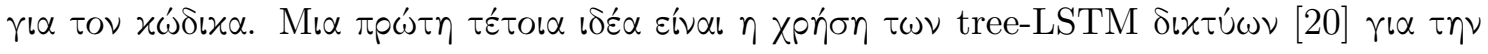

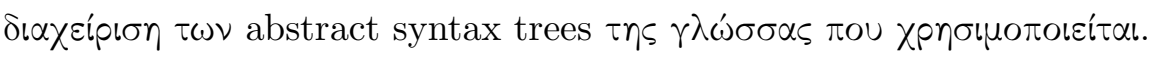

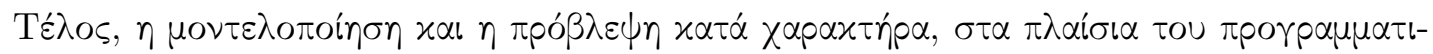

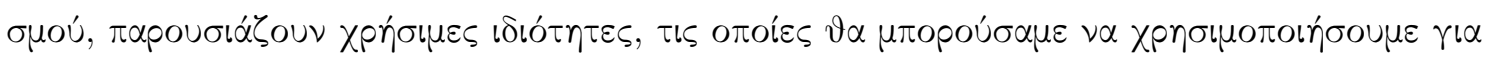

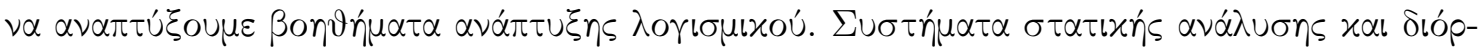

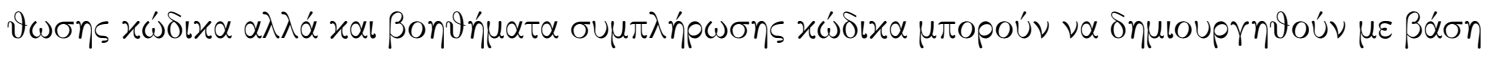

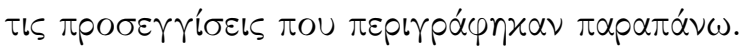






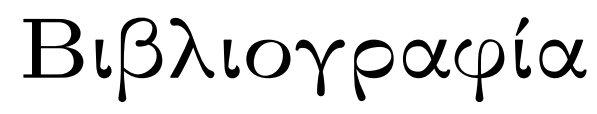

[1] D. Bahdanau, K. Cho, and Y. Bengio. Neural Machine Translation by Jointly Learning to Align and Translate. ICLR, 2014.

[2] J. Bergstra and Y. Bengio. Random Search for Hyper-Parameter Optimization. Journal of Machine Learning Research, 2012.

[3] A. W. Biermann. Automatic programming : A tutorial on formal methodologies. Journal of Symbolic Computation, 1985.

[4] I. Goodfellow, J. Pouget-Abadie, M. Mirza, D. Warde-Farley, S. Ozair, A. Courville, and Y. Bengio. Generative Adversarial Nets. NIPS, 2014.

[5] A. Graves. Generating Sequences with Recurrent Neural Networks. Technical Reports, 2013.

[6] S. Hochreiter and J. J. Schmidhuber. Long short-term memory. Neural Computation, 1997.

[7] A. Joulin and T. Mikolov. Inferring Algorithmic Patterns with Stack-Augmented Recurrent Nets. arXiv, 2015.

[8] Y. LeCun, Y. Bengio, and G. Hinton. Deep learning. Nature, 2015.

[9] P. Liu, X. Qiu, and X. Huang. Recurrent Neural Network for Text Classification. $\operatorname{arXiv,~} 2016$.

[10] V. Murali, S. Chaudhuri, and C. Jermaine. Bayesian Sketch Learning for Program Synthesis. arXiv, 2017.

[11] E. Parisotto, A.-R. Mohamed, R. Singh, L. Li, D. Zhou, and P. Kohli. Neuro-Symbolic Program Synthesis. arXiv, 2017.

[12] D. L. Parnas. Software aspects of strategic defense systems. ACM SIGSOFT Software Engineering Notes, 1985.

[13] R. Pascanu, T. Mikolov, and Y. Bengio. On the difficulty of training Recurrent Neural Networks. arXiv, 2012. 
[14] D. C. Schmidt. Model-Driven Engineering. IEEE Computer, 2006.

[15] N. Srivastava, G. Hinton, A. Krizhevsky, I. Sutskever, and R. Salakhutdinov. Dropout: A Simple Way to Prevent Neural Networks from Overfitting. Journal of Machine Learning Research, 2014.

[16] S. Sukhbaatar, A. Szlam, J. Weston, and R. Fergus. End-To-End Memory Networks. NIPS, 2015.

[17] I. Sutskever. Training Recurrent neural Networks. PhD thesis, 2013.

[18] I. Sutskever, J. Martens, and G. Hinton. Generating Text with Recurrent Neural Networks. ICML, 2011.

[19] I. Sutskever, O. Vinyals, and Q. V. Le. Sequence to sequence learning with neural networks. NIPS, 2014.

[20] K. S. Tai, R. Socher, and C. D. Manning. Improved Semantic Representations From Tree-Structured Long Short-Term Memory Networks. ACL, 2015.

[21] P. Yin and G. Neubig. A Syntactic Neural Model for General-Purpose Code Generation. arXiv, 2017. 
